Supporting Information

\title{
Protonation/Reduction of Carbonyl-Rich Diiron Complexes and the Direct Observation of Triprotonated Species: Insights into the Electrocatalytic Mechanism of Hydrogen Formation
}

Yu-Chiao Liu, ${ }^{\dagger}$ Kai-Ti Chu, ${ }^{\dagger}, \AA$ Yi-Lan Huang, ${ }^{\dagger}$ Cheng-Huey Hsu, ${ }^{\dagger}$ Gene-Hsiang Lee, $\|$ MeiChun Tseng, ${ }^{\dagger}$ Ming-Hsi Chiang $*, \dagger$,

†nstitute of Chemistry, Academia Sinica, Nankang, Taipei 115, Taiwan.

${ }^{+}$Molecular Science and Technology Program, TIGP, Institute of Chemistry, Academia Sinica, Nankang, Taipei 115, Taiwan

$\S$ Department of Chemistry, National Tsing Hua University, Hsinchu 300, Taiwan

Instrumentation Center, National Taiwan University, Taipei 106, Taiwan

*To whom correspondence should be addressed. E-mail: mhchiang@chem.sinica.edu.tw. 


\section{Contents}

\section{Schemes}

Scheme S1. Reaction schemes involving the " $2 \mathrm{H}$ " species.

Scheme S2. Reaction schemes involving the " $3 \mathrm{H}$ " species.

\section{Figures}

Figure S1. In-situ FTIR spectra of $1 \mu \mathbf{H}^{+}, \mathbf{1} \mu \mathrm{H}$, and $1 \mu \mathbf{H}^{*-}$.

Figure S2. High-field ${ }^{1} \mathrm{H}-\mathrm{NMR}$ spectra of $\mathbf{1} \boldsymbol{\mu} \mathbf{H}^{+}-\mathbf{3} \boldsymbol{\mu} \mathbf{H}^{+}$.

Figure S3. Variable temperature ${ }^{13} \mathrm{C}-\mathrm{NMR}$ spectra of $2 \boldsymbol{\mu} \mathbf{H}^{+}$.

Figure S4. Molecular structure of $1 \mu \mathbf{H}^{+}$.

Figure S5. Molecular structure of $\mathbf{3} \boldsymbol{\mu} \mathbf{H}^{+}$.

Figure S6. Cyclic voltammograms of $\mathbf{1} \boldsymbol{\mu} \mathbf{H}^{+}-\mathbf{3} \boldsymbol{\mu} \mathbf{H}^{+}$and the comparison with the parent hexacarbonyl complexes.

Figure S7. Cyclic voltammograms of $\mathbf{1} \boldsymbol{\mu} \mathbf{H}^{+}-\mathbf{3} \boldsymbol{\mu} \mathbf{H}^{+}$with increments of $\left[\left(\mathrm{Et}_{3} \mathrm{Si}\right)_{2} \mathrm{H}\right]\left[\mathrm{B}\left(\mathrm{C}_{6} \mathrm{~F}_{5}\right)_{4}\right]$.

Figure S8. Electronic spectra of $1 \mu \mathbf{H}^{+}, \mathbf{2} \mu \mathrm{H}^{+}, \mathbf{3} \mu \mathbf{H}^{+}, 3$ and $\mathbf{3} \mu \mathrm{H}^{+}$upon one-electron reduction.

Figure S9. CW X-band EPR spectrum of $3 \boldsymbol{\mu H}$ at $77 \mathrm{~K}$.

Figure S10. The calculated frequencies and vertical electronic transitions of $\mathbf{3}, \mathbf{3 \mu \mathbf { H } ^ { + }}$ and $3 \mu \mathbf{H}^{*}$.

Figure S11. Variable-temperature ${ }^{2} \mathrm{D}$ and ${ }^{1} \mathrm{H}$ NMR spectra of $\mathbf{2} \boldsymbol{\mu H S D} *$.

Figure S12. Positive ESI-MS spectra of $2 \boldsymbol{\mu H} \mathrm{HDSD}^{*+}$.

Figure S13. Molecular structure of $[2 \mu \mathrm{H}][\mathrm{OTf}]$.

Figure S14. Computed structures of the $C E$ species for the pdt analogs.

Figure S15. HOMO and orbital contribution analysis of $2 \mu \mathbf{H}^{*-}$.

Figure S16. Optimized structures of the triprotonated cationic and neutral species.

Figure S17. Comparison of experimental and calculated $\mathrm{CO}$ frequencies of the reduced/protonated derivatives of complex 2 .

Figure S18. Comparison of experimental and calculated CO frequencies of all possible reduced/protonated derivatives of complex 2 . 
Figure S19. The linear relationship of experimental and calculated CO frequencies using B3LYP functional of complex 2 and its reduced/protonated derivatives.

Figure S20. The linear relationship of experimental and calculated $\mathrm{CO}$ frequencies using BP86 functional of complex 2 and its reduced/protonated derivatives.

Figure S21. The two dimensional potential energy surface showing the transition from $2 \mu \mathrm{HSH}^{\mathrm{in} *}$ to $2 \mathrm{tHSH}^{\mathrm{in} *}$.

Figure S22. Time-dependent in-situ FTIR spectra of the conversion from $2 \boldsymbol{\mu H S H} *$ (blue line) to 2 (red line) in $\mathrm{CH}_{2} \mathrm{Cl}_{2}$ solution at $213 \mathrm{~K}$.

Figure S23. Chemdraw plots of the optimized structures of the intermediate species $\left(\mathbf{2} \mathbf{t H t H}_{2}{ }^{\text {in }{ }^{++}}, \mathbf{2 t H}_{2}{ }^{\text {in }} \mathbf{S H}^{\text {in } *^{+}}, \mathbf{2 t H}_{2}{ }^{\text {in }} \mathbf{S H}^{*+}\right)$ in hydrogen evolution involving $\mathbf{2} \boldsymbol{\mu H} \mathbf{H H}^{\mathbf{s}} \mathbf{S H}^{*+}$.

Figure S24. Optimized structures of the diprotonated species and its two subsequent reduced products.

\section{Tables}

Table S1. X-ray crystallographic data.

Table S2. Selected bond distances $(\AA)$ and angles $\left(^{\circ}\right)$ of the crystallographic data and the DFT geometry-optimized models for $\mathbf{1} \boldsymbol{\mu} \mathbf{H}^{+}, \mathbf{2} \boldsymbol{\mu} \mathbf{H}^{+}$and $\mathbf{3} \boldsymbol{\mu} \mathbf{H}^{+}$.

Table S3. Calculated $\Delta \mathrm{p} K_{\mathrm{a}}$ values and reduction potentials $\left(E^{\circ} v s . \mathrm{Fc}^{+} / \mathrm{Fc}\right)$ with solvation $\left(\mathrm{CH}_{2} \mathrm{Cl}_{2}\right)$ correction of the protonated as well as reduced species of complex 2.

Table S4. Estimated $\mathrm{p} K_{\mathrm{a}}$ values from the calculation with solvation $\left(\mathrm{CH}_{2} \mathrm{Cl}_{2}\right)$ correction of the protonated as well as reduced species of complex 2 .

Table S5. Thermodynamic relationships and equations for theoretical estimation of the reduction potential.

Table S6. Summary of solvent corrected $v$ CO IR spectra for proposed intermediates related to complex 2 and imaginary frequencies for the transition states.

Table S7. Summary of solvent corrected free energy for all computed species and transition states.

Table S8. Summary of solvent corrected free energy for complex 2 and its related protonated/reduced species computed using BP86 functional.

Table S9. Cartesian coordinates for DFT geometry-optimized species using B3LYP.

Table S10. Cartesian coordinates for DFT geometry-optimized species using BP86.

\section{References}




\section{Schemes}

In the first $\mathrm{H}_{2}$ evolution process via $2 \mu \mathbf{H S H}^{*}$ (the " $2 \mathrm{H}$ " species), the rate-determining step is $\mathbf{2} \boldsymbol{\mu} \mathbf{H S H}^{\mathrm{in} *} \rightarrow \mathbf{2 t H}_{2}{ }^{*}$, based on the high activation energy; it is achieved due to the acidic character of the $\mu-\mathrm{H}$ group in the " $2 \mathrm{H}$ " species, which does not react with HOTf to form $\mathrm{H}_{2}$ in solution. The free energy profile concerning this pathway is displayed in Scheme S1.

Scheme S1. Free energy profile for hydrogen formation from $2 \mu \mathbf{H S H}^{*}$ to 2 via $2 \mathbf{t H}_{2} *$.

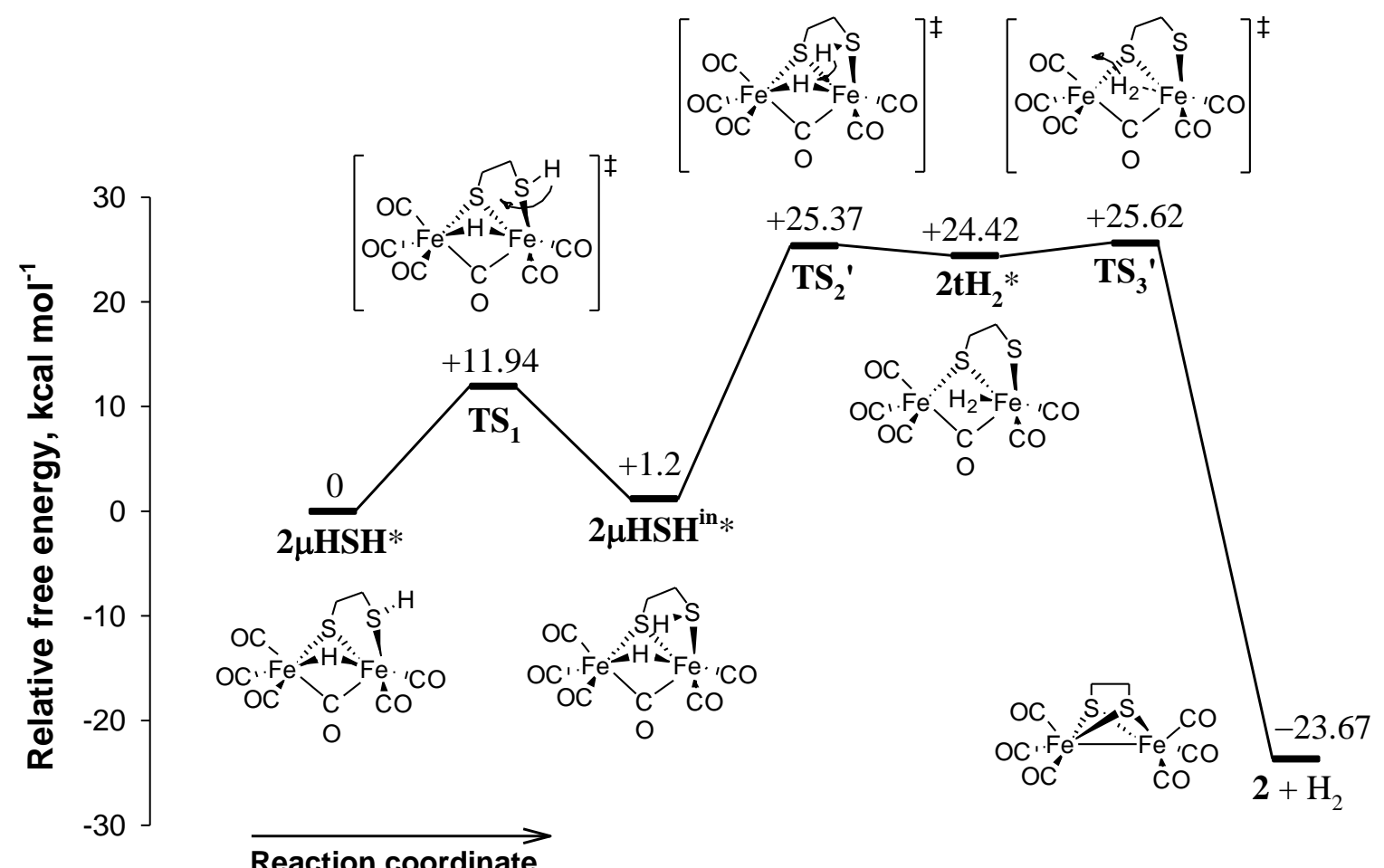

In the $\mathrm{H}_{2}$ evolution process via $2 \boldsymbol{\mu} \mathbf{H} \mathbf{t H}^{\mathrm{s}} \mathbf{S H} \mathbf{H}^{*+}$ (the " $3 \mathrm{H}^{\prime}$ " species), the key intermediate is the $\eta^{2}-\mathrm{H}_{2}$ adduct. The first type of plausible $\eta^{2}-\mathrm{H}_{2}$ adduct species were $\mathbf{2} \boldsymbol{\mu} \mathbf{H t H}_{2}{ }^{\text {in } *+}$ and $\mathbf{2 t H t H}_{2}{ }^{\text {in } *^{+}}$, which each consisted of a dihydrogen molecule inside the $\mathrm{FeS}^{\mu} \mathrm{FeS}^{\mathrm{t}} \mathrm{H}^{\mu}$ packet and close to the hydride, as shown in Figures 15 and S23. The second class had similar $\eta^{2}-\mathrm{H}_{2}$ features 
and included $\mathbf{2} \mathbf{2} \mathbf{H}_{2}{ }^{\mathrm{in}} \mathbf{S H}^{\mathrm{in}} *^{++}$and $\mathbf{2} \mathbf{\mathbf { t }} \mathbf{H}_{2}{ }^{\mathrm{in}} \mathbf{S H}^{*+}$, which were differentiated from the syn/anti orientation of $\mathrm{S}^{\mathrm{t}}-\mathrm{H}$ with respect to $\eta^{2}-\mathrm{H}_{2}$. The optimization of these four species eventually converged back to the corresponding initial structures without the $\eta^{2}-\mathrm{H}_{2}$ adduct, except for $\mathbf{2} \boldsymbol{\mu H t H} \mathbf{H}^{\text {in } *+}$. The formation of $\mathbf{2} \mu \mathbf{H t H}_{2}{ }^{\text {in } *+}$ arose from bond formation between $\mathrm{t}-\mathrm{H}$ and $S-\mathrm{H}$ within the $\mathrm{FeS}^{\mu} \mathrm{FeS}^{t} \mathrm{H}^{\mu}$ packet. These results were consistent with the recognized statement that $\mathrm{t}-$ $\mathrm{H}$ is of comparatively higher hydricity and more reactive towards the acidic $S-\mathrm{H}$ than the $\mu-\mathrm{H}$.

\section{Alternative mechanism for $\mathrm{H}_{2}$ evolution via $2 \mu \mathrm{HtH}^{\mathrm{s} S H}{ }^{*+}$ (the "3H" species). Three} additional mechanistic routes are also investigated by DFT calculation. They are used for comparison with the proposed one discussed in the main text. According to the energy barriers in the mechanistic pathways, the mechanisms in the supporting information section are less likely. They are discussed below.

The formation of the $\eta^{2}-H_{2}$ adduct. In addition to the cases exhibiting the $\eta^{2}-\mathrm{H}_{2}$ group inside the $\mathrm{FeS}^{\mu} \mathrm{FeS} \mathrm{H}^{\mu}$ packet, the third scenario is to place the $\eta^{2}-\mathrm{H}_{2}$ group outside the $\mathrm{FeS}{ }^{\mu} \mathrm{FeS} \mathrm{S}^{\mathrm{t}} \mathrm{H}^{\mu}$ packet. It has been shown that 2 stereoisomers, $\mathbf{2} \boldsymbol{\mu} \mathbf{H t H}^{\mathrm{s}} \mathbf{S H} \mathbf{H}^{*+}$ and $\mathbf{2} \boldsymbol{\mu} \mathbf{H} \mathbf{t H} \mathbf{S H}^{*+}$, are almost isoenergetic. We have found out that the tautomerization between two species is a slow process with $\Delta \Delta G\left(\mathbf{T S}_{7}\right)=+21.21 \mathrm{kcal} \mathrm{mol}^{-1}$. The subsequent generation of $\mathbf{2} \mathbf{t} \mathbf{H}_{2} \mathbf{S} \mathbf{H}^{*+}$ from $\mathbf{2} \boldsymbol{\mu} \mathbf{H} \mathbf{t} \mathbf{H}^{\mathrm{a}} \mathbf{S} \mathbf{H}^{*+}$ requires a lower energy barrier $\left(\mathbf{T S}_{8}\right)$ of $+15.33 \mathrm{kcal} \mathrm{mol}^{-1}$. It is found that the resulting fifth $\eta^{2}$ $\mathrm{H}_{2}$ species, $\mathbf{2} \mathbf{t H}_{2} \mathbf{S H}^{*+}$, is of an identical free energy as $\mathbf{2} \mathbf{t H}_{2} \mathbf{S} \mathbf{H}^{\mathbf{i n} *+}$, the sixth analog. The only structural difference is derived from the position of the $S^{t}$-proton in which it is located outside and inside the packet in $\mathbf{2} \mathbf{t} \mathbf{H}_{2} \mathbf{S} \mathbf{H}^{*+}$ and $\mathbf{2} \mathbf{2} \mathbf{H}_{2} \mathbf{S} \mathbf{H}^{\mathrm{in} *+}$, respectively.

The replenishment of $2 \mu H^{+}$to close the catalytic cycle. Regeneration of $\mathbf{2} \boldsymbol{\mu} \mathbf{H}^{+}$from $\mathbf{2} \mathbf{t} \mathbf{H}_{2} \mathbf{S} \mathbf{H}^{\text {in } *+}$ or $\mathbf{2} \mathbf{t H}_{2} \mathbf{S H}^{*+}$ involves 2 steps: release of molecule $\mathrm{H}_{2}$ and proton migration. Red lines in Scheme 
S1 represent the possible pathways concerning $2 \mathbf{t H}_{2} \mathbf{S} \mathbf{H}^{\mathbf{i n} *+}$ with the initial $\mathrm{H}_{2}$ evolution followed by proton transfer to the $\mathrm{Fe}_{2}$ core. The possible pathways with the reverse steps are depicted as blue lines. The green lines represent the possible pathways initiated by $\mathrm{H}_{2}$ release from $\mathbf{2} \mathbf{t H}_{2} \mathbf{S H}^{*+}$, followed by the conversion to $\mathbf{2} \mathbf{S} \mathbf{H}^{\text {in } *+}$. As observed, the most energy demanding step corresponds to the formation of the $\{\mathrm{Fe}(\mu-\mathrm{H}) \mathrm{Fe}\}$ core due to proton transfer from the $\mathrm{S}^{\mathrm{t}}-\mathrm{H}$ site. Such high energy barrier $\left(\Delta \Delta G\left(\mathbf{T S}_{11}\right), \Delta \Delta G\left(\mathbf{T S}_{12}\right)>+22 \mathrm{kcal} \mathrm{mol}^{-1}\right)$ is realized by low acidity of the $\mathrm{S}^{\mathrm{t}}-\mathrm{H}$ group relative to $\left[\left(\mathrm{Et}_{3} \mathrm{Si}\right)_{2} \mathrm{H}\right]\left[\mathrm{B}\left(\mathrm{C}_{6} \mathrm{~F}_{5}\right)_{4}\right]$. Protonation onto the $\mathrm{Fe}_{2}$ core thus is assumed to be the last favored step. The $\eta^{2}-\mathrm{H}_{2}$ species readily undergoes release of $\mathrm{H}_{2}$ as small energy barriers $\left(\approx+3 \mathrm{kcal} \mathrm{mol}^{-1}\right)$ are estimated. In addition, the resulting complexes are thermodynamically more stable than the corresponding $\eta^{2}-\mathrm{H}_{2}$ species. It is said that the $\eta^{2}-\mathrm{H}_{2}$ adduct is short-lived for prompt $\mathrm{H}_{2}$ dissociation. This proposed mechanism involves several energetically high-lying intermediates and transition states. It reasonably explains why the slower $\mathrm{H}_{2}$ evolution process via $\mathbf{2} \boldsymbol{\mu} \mathbf{H t} \mathbf{H}^{\mathrm{s}} \mathbf{S} \mathbf{H}^{*+}$ was observed in experiments. In these pathways (the green, blue and red versions), $\mathrm{H}_{2}$ evolution and proton migration are discussed separately. It is also possible for both steps to take place concomitantly, which could not be rules out, since the distorted square-pyramidal geometry about the Fe centers in $\mathbf{2} \mathbf{t} \mathbf{H}_{2} \mathbf{S} \mathbf{H}^{\mathbf{i n} *+}$ and $\mathbf{2} \mathbf{t} \mathbf{H}_{2} \mathbf{S H}{ }^{*+}$ is prone to ligand attack. 
Scheme S2. Free energy profile (top) and mechanistic cycle of postulated pathways (bottom) for hydrogen formation of $\mathbf{2} \boldsymbol{\mu} \mathbf{H t H} \mathbf{H}^{\mathrm{s}} \mathbf{S} \mathbf{H}^{*+}$ to $\mathbf{2} \boldsymbol{\mu} \mathbf{H}^{+}$. The red, blue and green lines represent the different pathways.

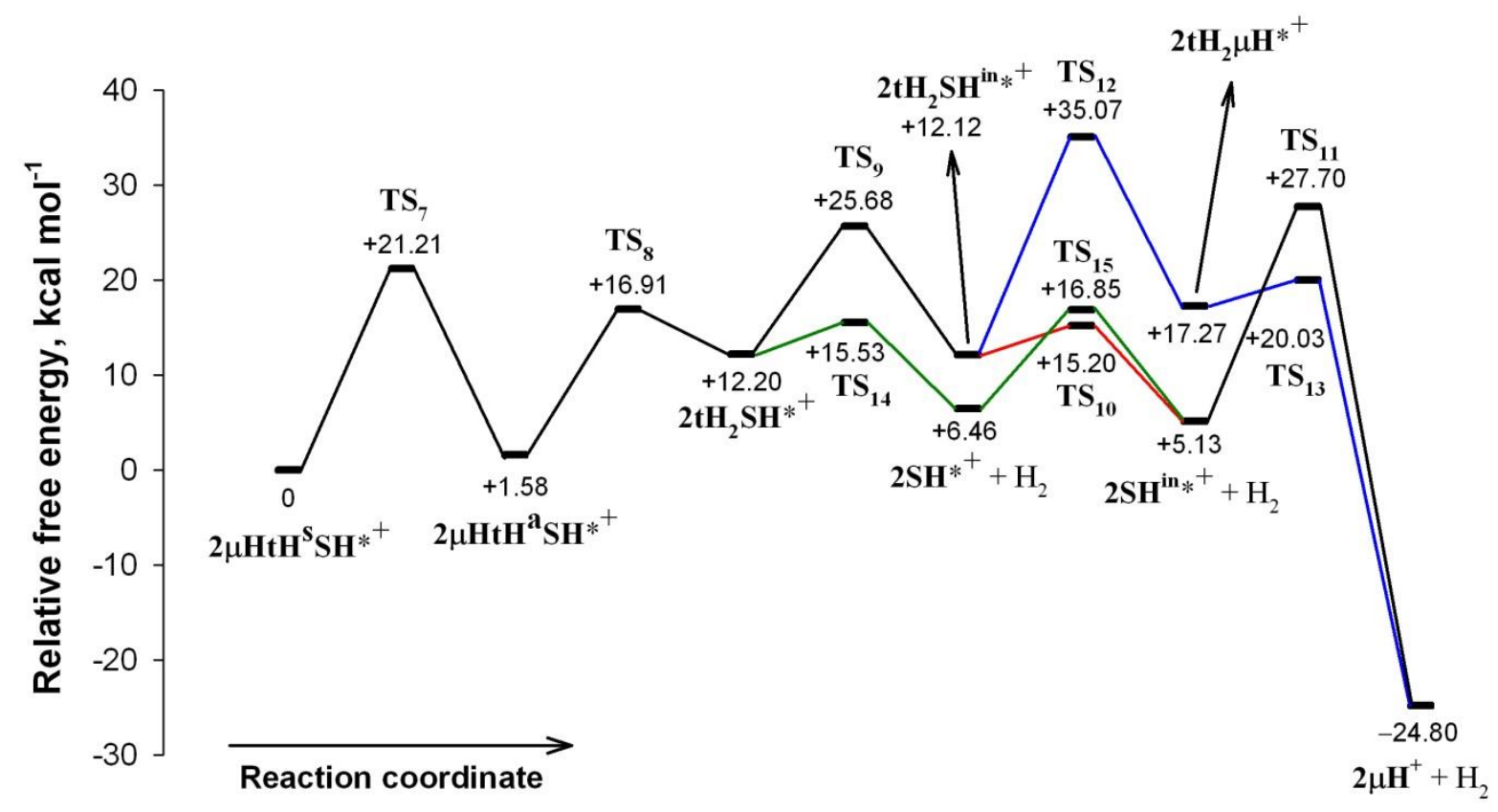




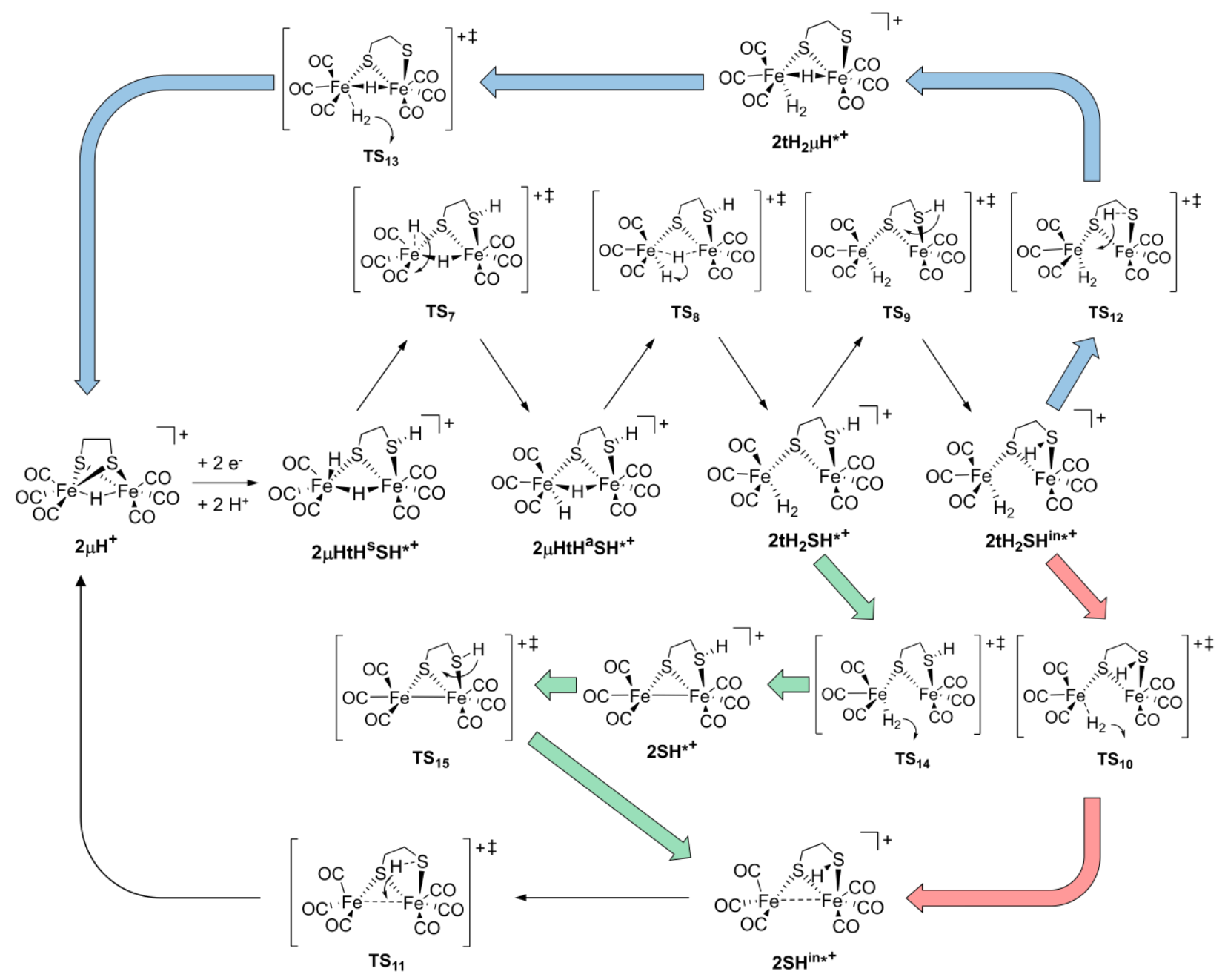


Figures

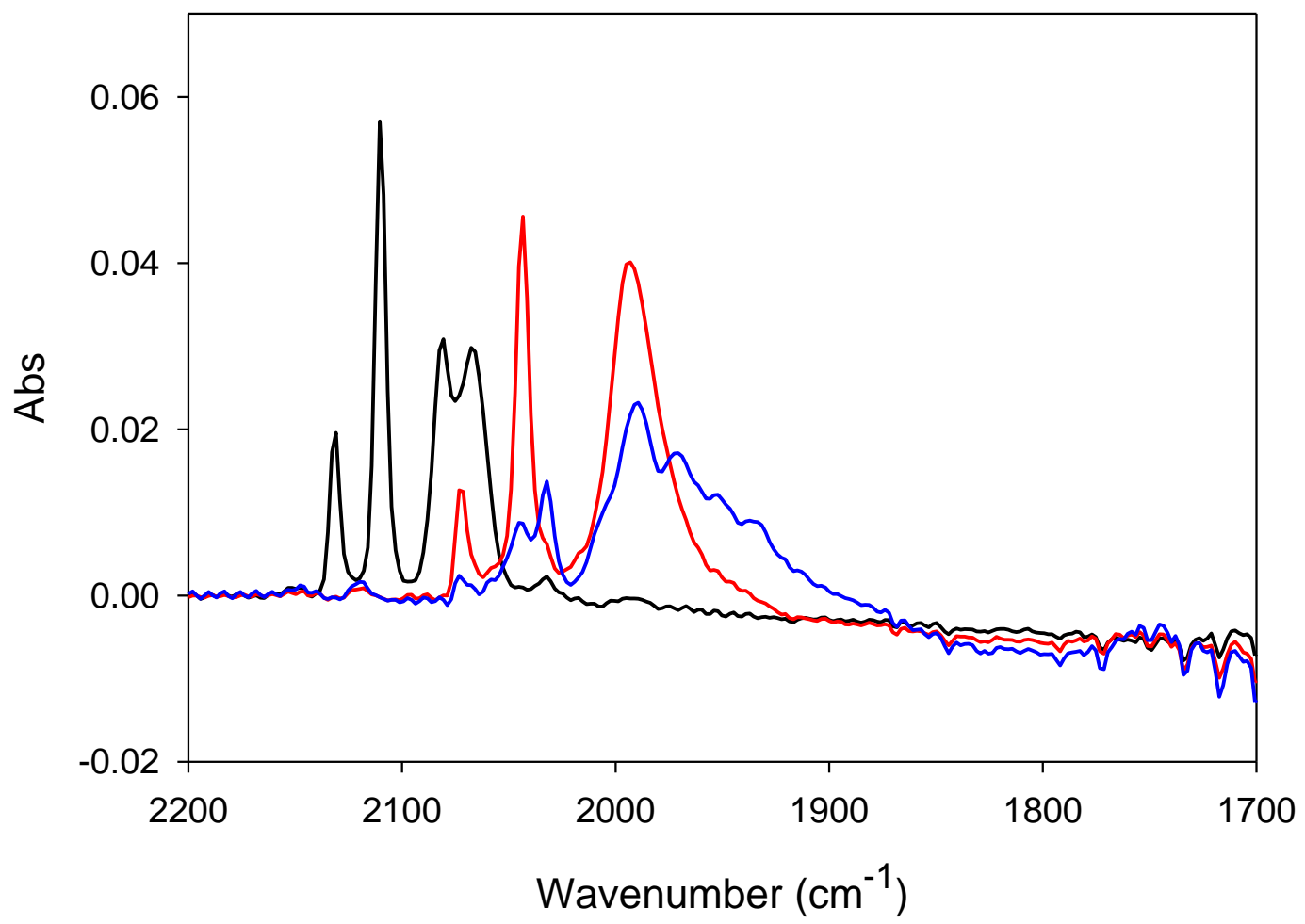

Figure S1. In-situ FTIR spectra of $1 \mu \mathbf{H}^{+}$(black), $1 \boldsymbol{\mu H}$ (red), and $1 \boldsymbol{\mu} \mathbf{H}^{*^{-}}$(blue) in $\mathrm{CH}_{2} \mathrm{Cl}_{2}$ solution at $193 \mathrm{~K}$. 


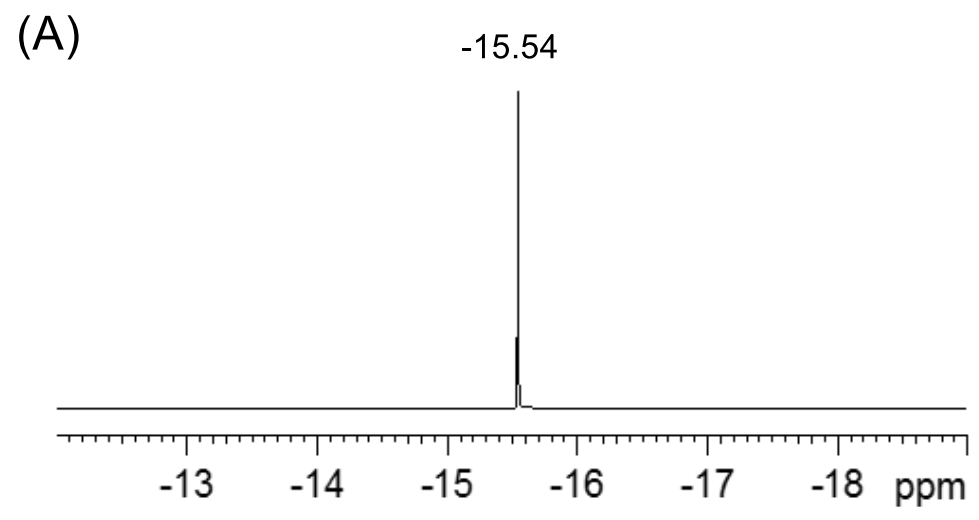

(B)

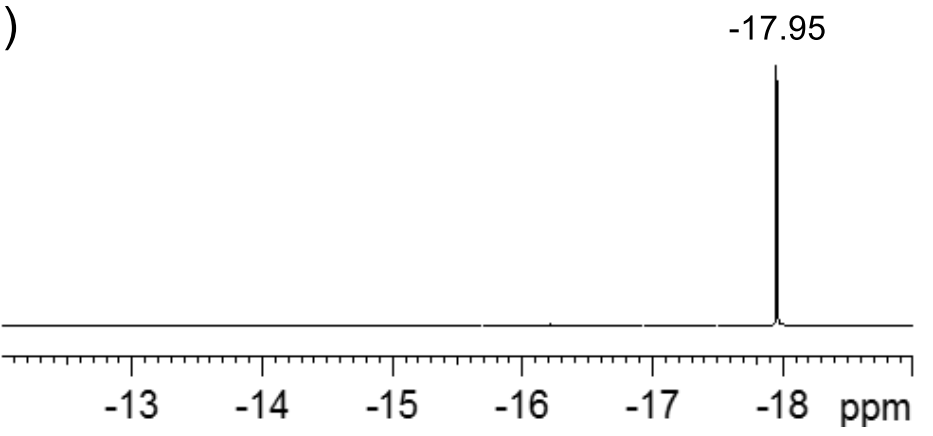

$-13.40$

(C)

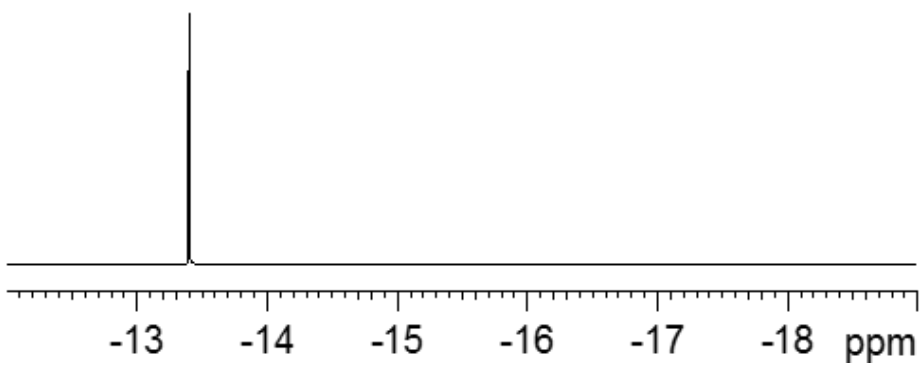

Figure S2. High-field ${ }^{1} \mathrm{H}-\mathrm{NMR}$ spectra of (A) $\mathbf{1} \boldsymbol{\mu} \mathbf{H}^{+}$(B) $\mathbf{2} \boldsymbol{\mu} \mathbf{H}^{+}$(C) $\mathbf{3} \boldsymbol{\mu} \mathbf{H}^{+}$in $\mathrm{CD}_{2} \mathrm{Cl}_{2}$ solution. 
$298 \mathrm{~K}$

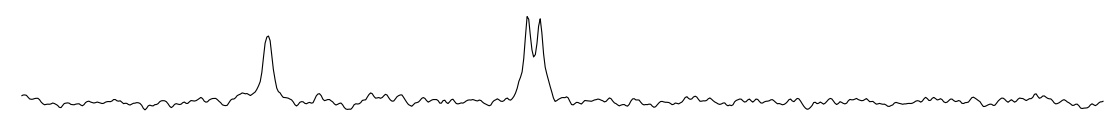

$253 \mathrm{~K}$

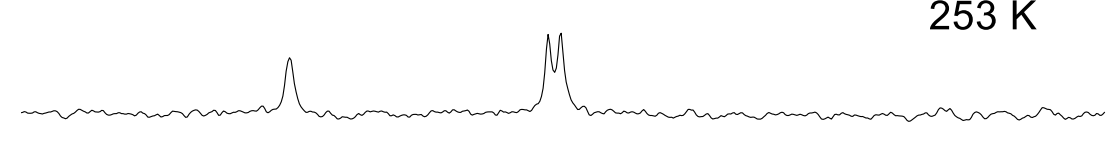

$233 \mathrm{~K}$
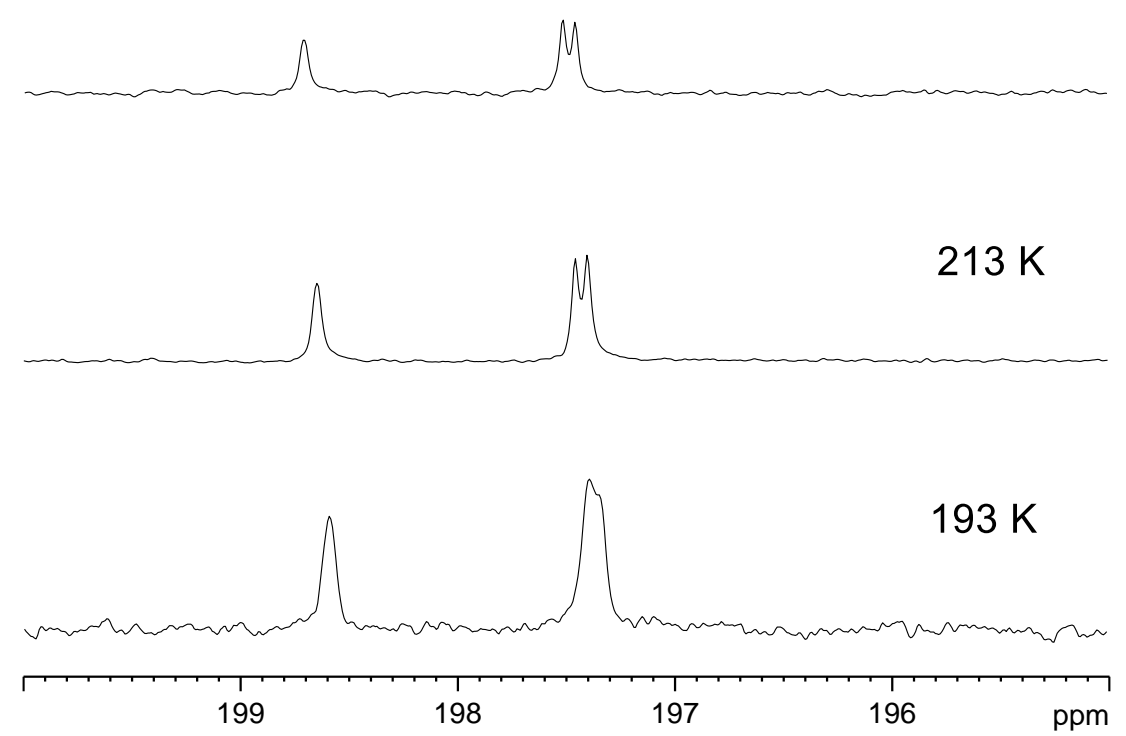

Figure S3. Variable temperature ${ }^{13} \mathrm{C}-\mathrm{NMR}$ spectra of $2 \boldsymbol{\mu} \mathbf{H}^{+}$in the downfield region. 


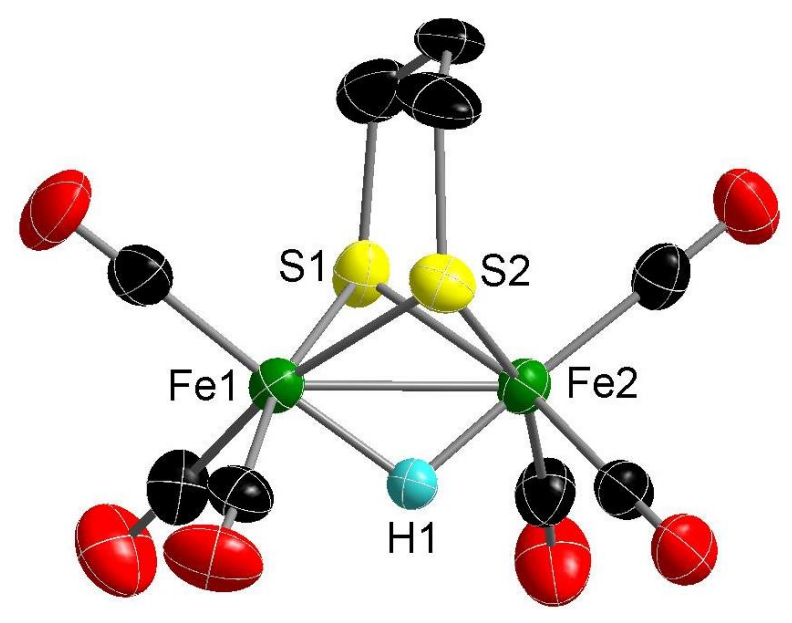

Figure S4. Molecular structure of $\mathbf{1} \boldsymbol{\mu} \mathbf{H}^{+}$. All hydrogen atoms except for the hydride are omitted for clarity. Selected bond length $(\AA)$ and angles (deg): $\mathrm{Fe}-\mathrm{Fe}, 2.5641(10) ; \mathrm{Fe}-\mathrm{S}$, ave. 2.2601(15); $\mathrm{Fe}-\mathrm{C}_{\mathrm{CO}, a p}$, ave. 1.806(6); Fe-Cco,ba, ave. 1.823(6); Fe1-H1, 1.70(6); Fe2-H1, 1.54(6); S-Fe-S, ave. 84.63(6); $\mathrm{S}-\mathrm{Fe}-\mathrm{Fe}$, ave. 55.44(4); $\mathrm{Fe}-\mathrm{S}-\mathrm{Fe}$, ave. 69.12(5).

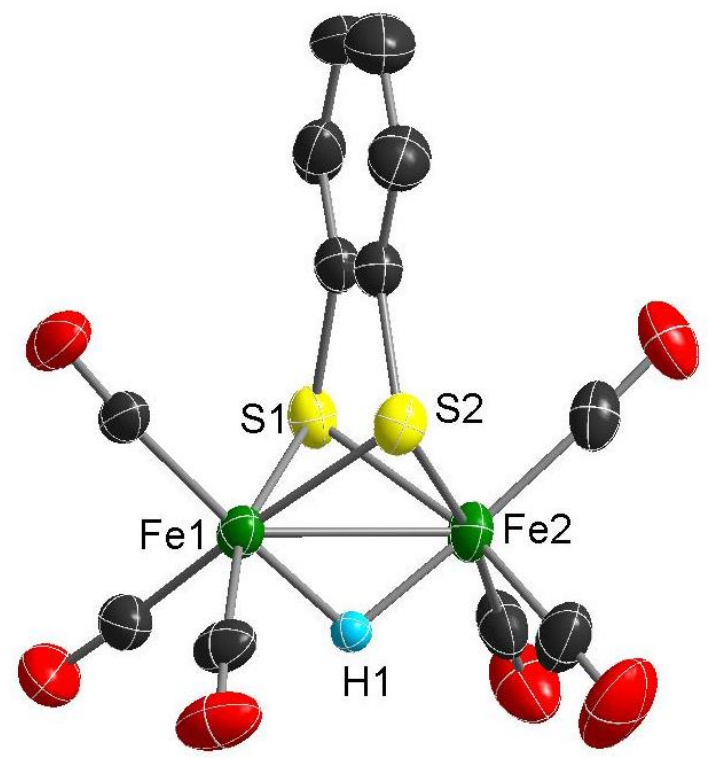

Figure S5. Molecular structure of $\mathbf{3} \boldsymbol{\mu} \mathbf{H}^{+}$. All hydrogen atoms except for the hydride are omitted for clarity. Selected bond length ( $\mathrm{A}$ ) and angles (deg): $\mathrm{Fe}-\mathrm{Fe}, 2.5438$ (7); $\mathrm{Fe}-\mathrm{S}$, ave. 2.2700(11); $\mathrm{Fe}-\mathrm{C}_{\mathrm{CO}, a p}$, ave. 1.815(5); $\mathrm{Fe}-\mathrm{C}_{\mathrm{CO}, \text { ba, }}$ ave. 1.825(5); Fe1-H1, 1.63(4); Fe2-H1, 1.66(4); S-Fe-S, ave. 80.87(4); $\mathrm{S}-\mathrm{Fe}-\mathrm{Fe}$, ave. 55.92(3); $\mathrm{Fe}-\mathrm{S}-\mathrm{Fe}$, ave. 68.15(3). 

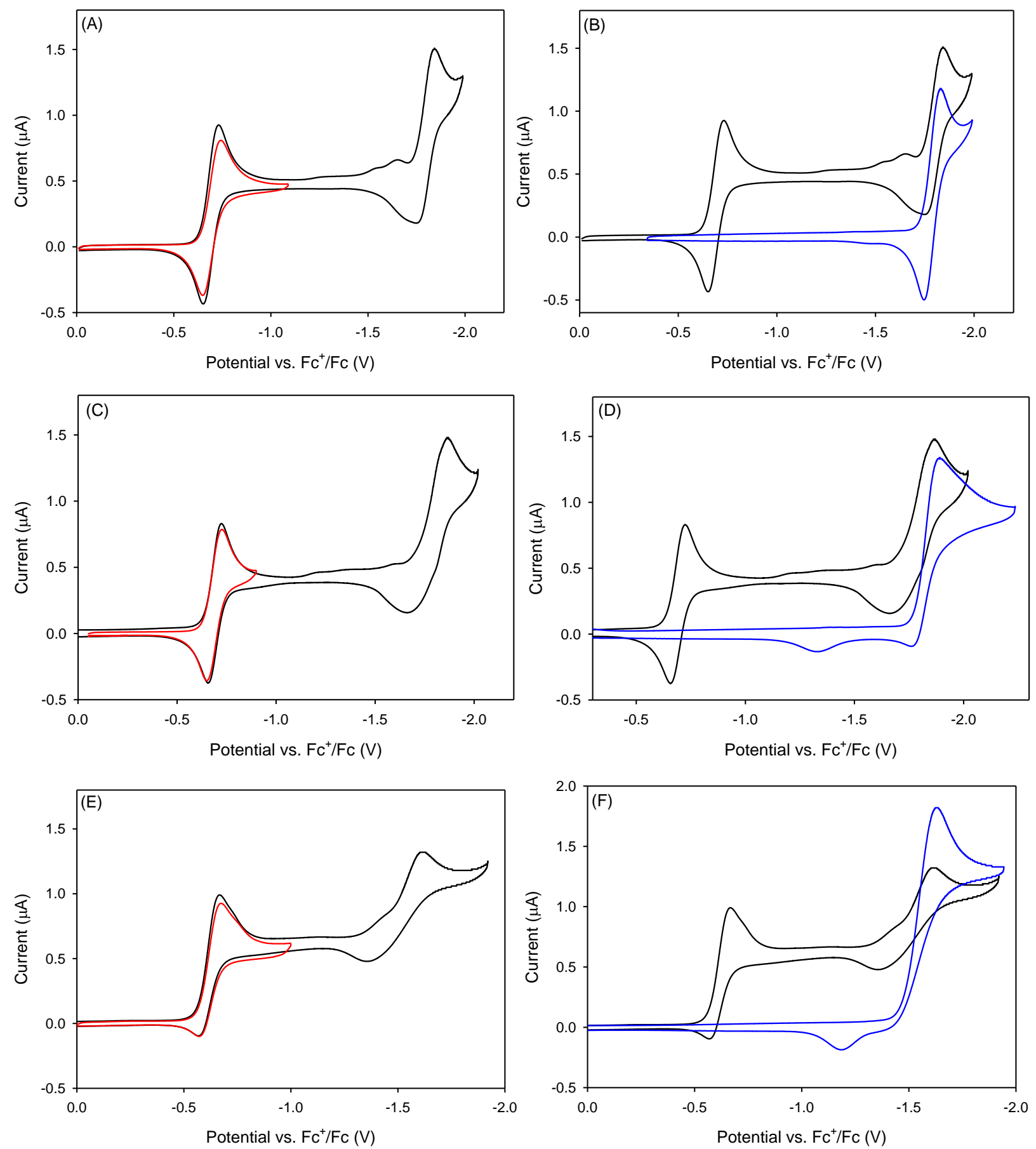

Figure S6. Cyclic voltammograms of $1 \boldsymbol{\mu} \mathbf{H}^{+}-\mathbf{3} \boldsymbol{\mu} \mathbf{H}^{+}$and the comparison with the parent hexacarbonyl complexes. (A) $\mathbf{1} \boldsymbol{\mu} \mathbf{H}^{+}(200 \mathrm{~K})$, (B) $\mathbf{1} \boldsymbol{\mu} \mathbf{H}^{+}$vs. [( $\left.\left.\mu-p d t\right) \mathrm{Fe}_{2}(\mathrm{CO})_{6}\right],(\mathrm{C}) \mathbf{2} \boldsymbol{\mu} \mathbf{H}^{+}(210 \mathrm{~K})$, (D) $\mathbf{2} \boldsymbol{\mu} \mathbf{H}^{+}$vs. $\left[\left(\mu\right.\right.$-edt) $\left.\mathrm{Fe}_{2}(\mathrm{CO})_{6}\right],(\mathrm{E}) \mathbf{3} \boldsymbol{\mu} \mathbf{H}^{+}(213 \mathrm{~K})$, (F) $\mathbf{3} \boldsymbol{\mu} \mathbf{H}^{+}$vs. [( $\mu$-bdt) $\left.\mathrm{Fe}_{2}(\mathrm{CO})_{6}\right]$. The black and red lines show the full and partial voltammograms, respectively. The blue line shows the voltammogram of the parent hexacarbonyl complex at $210 \mathrm{~K}$. Conditions: in $\mathrm{CH}_{2} \mathrm{Cl}_{2}$ solution, $\mathrm{N}_{2}$, [complex] $1 \mathrm{mM}, v=100 \mathrm{mV} \mathrm{s}^{-1}, 1 \mathrm{~mm}$ vitreous carbon electrode, $\left.0.1 \mathrm{M}[n-\mathrm{Bu} 4 \mathrm{~N}]\left[\mathrm{B}\left(\mathrm{C}_{6} \mathrm{~F}_{5}\right)_{4}\right]\right)$. 
Electrocatalytic reduction of $\left[\left(\mathrm{Et}_{3} \mathrm{Si}\right)_{2} \mathrm{H}\right]\left[\mathrm{B}\left(\mathrm{C}_{6} \mathrm{~F}_{5}\right)_{4}\right]$ by $\mathbf{1} \boldsymbol{\mu} \mathbf{H}^{+}-\mathbf{3} \boldsymbol{\mu} \mathbf{H}^{+}$was performed in $\mathrm{CH}_{2} \mathrm{Cl}_{2}$ solution under $\mathrm{N}_{2}$ at $298 \mathrm{~K}$. A catalytic respond was observed at the potential near the first reduction. The current intensity increased linearly with the acid concentration for $\mathbf{1} \boldsymbol{\mu} \mathbf{H}^{+}-\mathbf{3} \boldsymbol{\mu} \mathbf{H}^{+}$.
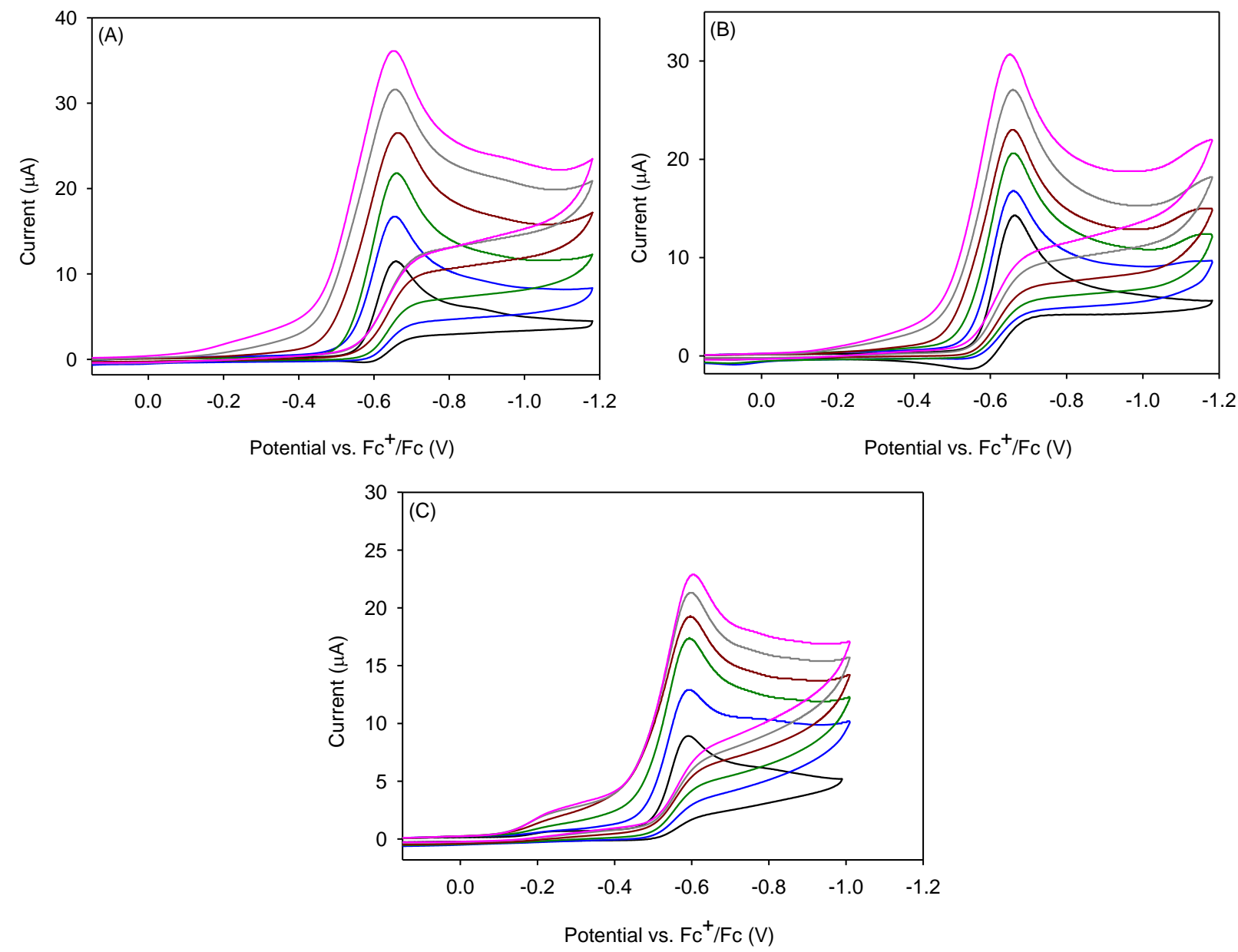

Figure S7. Cyclic voltammograms of (A) $1 \mu \mathbf{H}^{+}$(B) $\mathbf{2} \boldsymbol{\mu} \mathbf{H}^{+}$(C) $\mathbf{3} \boldsymbol{\mu} \mathbf{H}^{+}$with increments of $\left[\left(\mathrm{Et}_{3} \mathrm{Si}\right)_{2} \mathrm{H}\right]\left[\mathrm{B}\left(\mathrm{C}_{6} \mathrm{~F}_{5}\right)_{4}\right](0.0,1.0,2.0,3.0,4.0$, and 5.0 equiv. $)$ solution under $\mathrm{N}_{2}$ atmosphere ([complex] $1 \mathrm{mM}, v=100 \mathrm{mV} \mathrm{s}^{-1}, 3 \mathrm{~mm}$ vitreous carbon electrode, $0.1 \mathrm{M}[n$-Bu4 $\mathrm{N}]\left[\mathrm{B}\left(\mathrm{C}_{6} \mathrm{~F}_{5}\right)_{4}\right]$, $298 \mathrm{~K})$. 

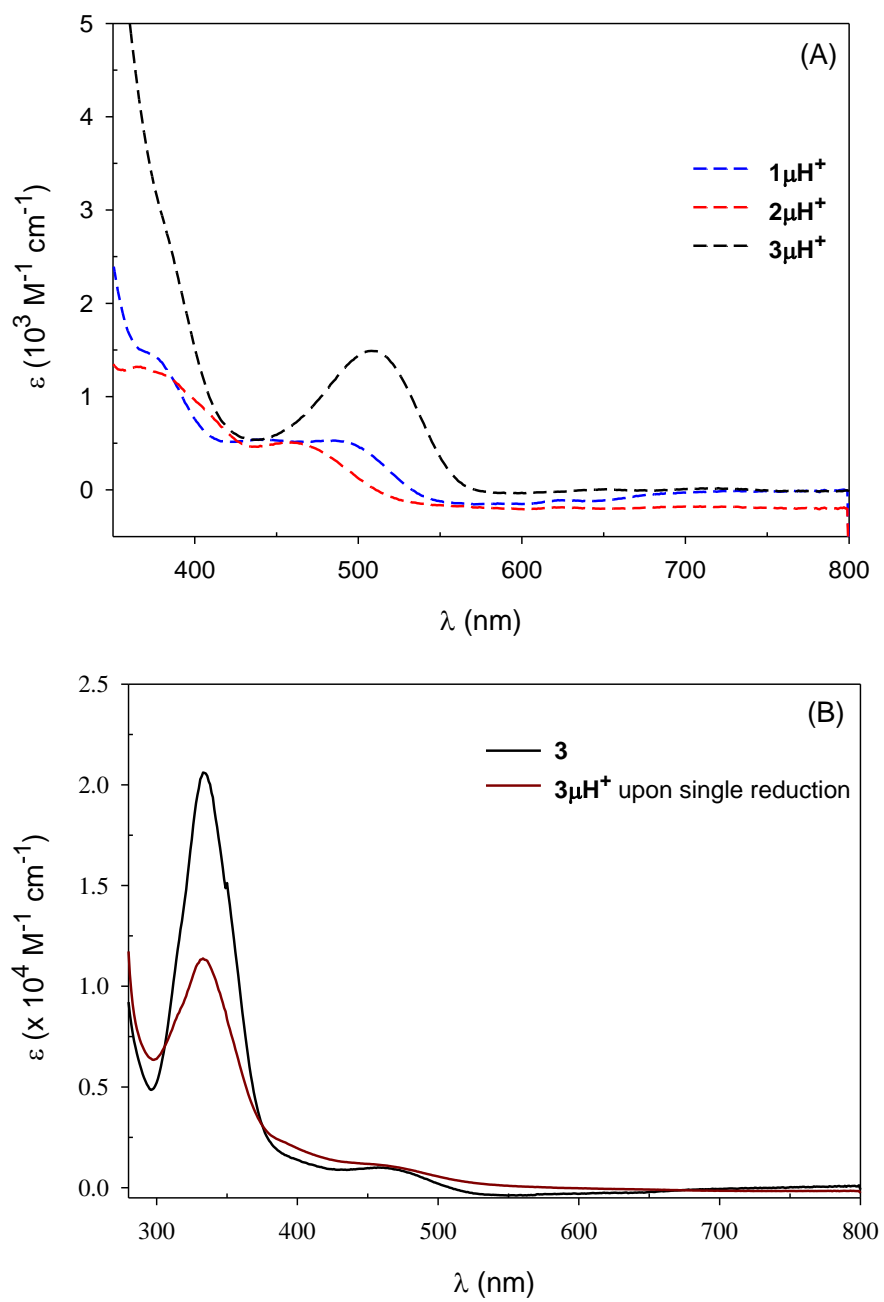

Figure S8. Electronic spectra of (A) $\mathbf{1} \boldsymbol{\mu} \mathbf{H}^{+}$(blue), $\mathbf{2} \boldsymbol{\mu} \mathbf{H}^{+}$(red) and $\mathbf{3} \boldsymbol{\mu} \mathbf{H}^{+}$(black) in $\mathrm{CH}_{2} \mathrm{Cl}_{2}$ solution at $195 \mathrm{~K}$, and (B) $\mathbf{3}$ (black) and $\mathbf{3} \boldsymbol{\mu} \mathbf{H}^{+}$upon single reduction (brick red) in $\mathrm{CH}_{2} \mathrm{Cl}_{2}$ solution at $193 \mathrm{~K}$ under $\mathrm{N}_{2}$ atmosphere.

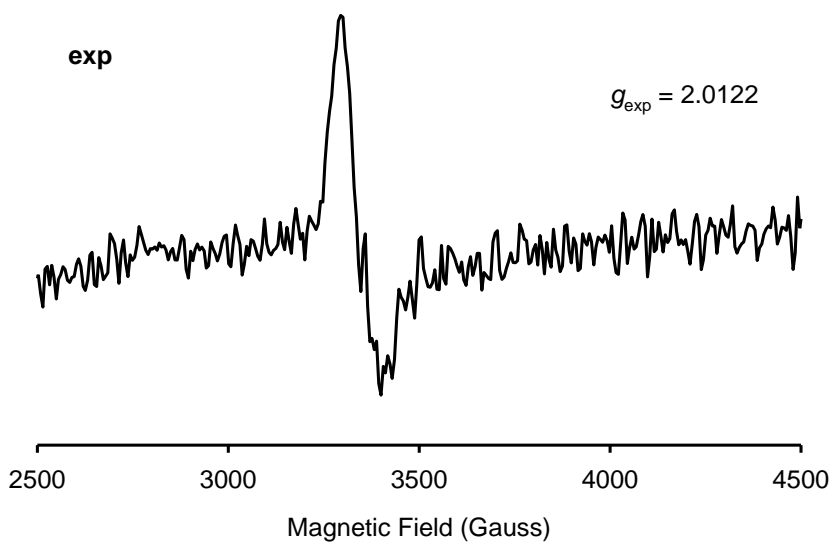

Figure S9. $\mathrm{CW}$ X-band EPR spectrum of $\mathbf{3} \boldsymbol{\mu} \mathbf{H}^{*}$ recorded in frozen $\mathrm{CH}_{2} \mathrm{Cl}_{2}$ solution at $77 \mathrm{~K}$. 

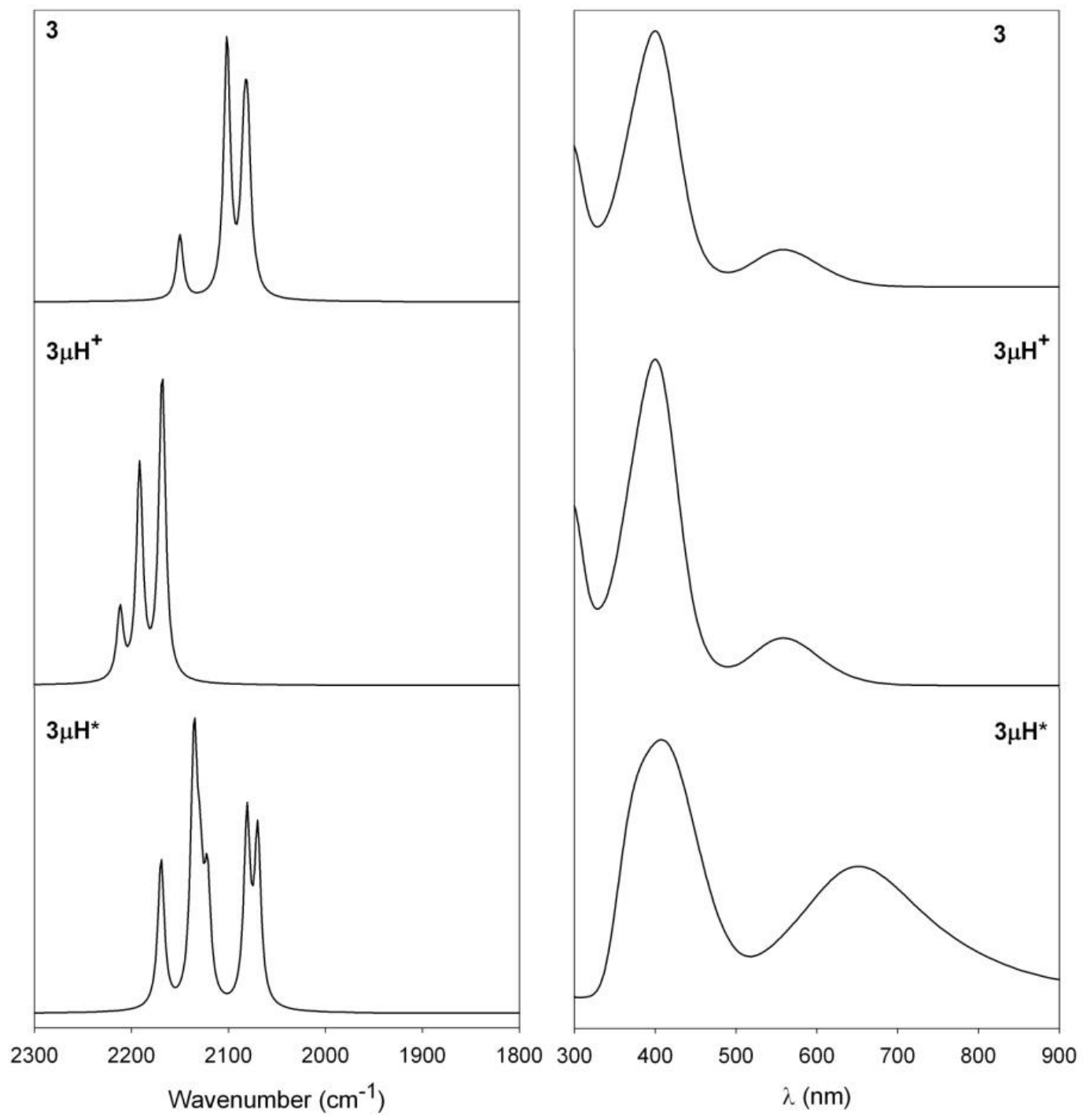

Figure S10. The calculated frequencies (left panel) and vertical electronic transitions (right panel) of the optimized structures of $\mathbf{3}, \mathbf{3} \boldsymbol{\mu} \mathbf{H}^{+}$and $\mathbf{3} \boldsymbol{\mu} \mathbf{H}^{*}$. 

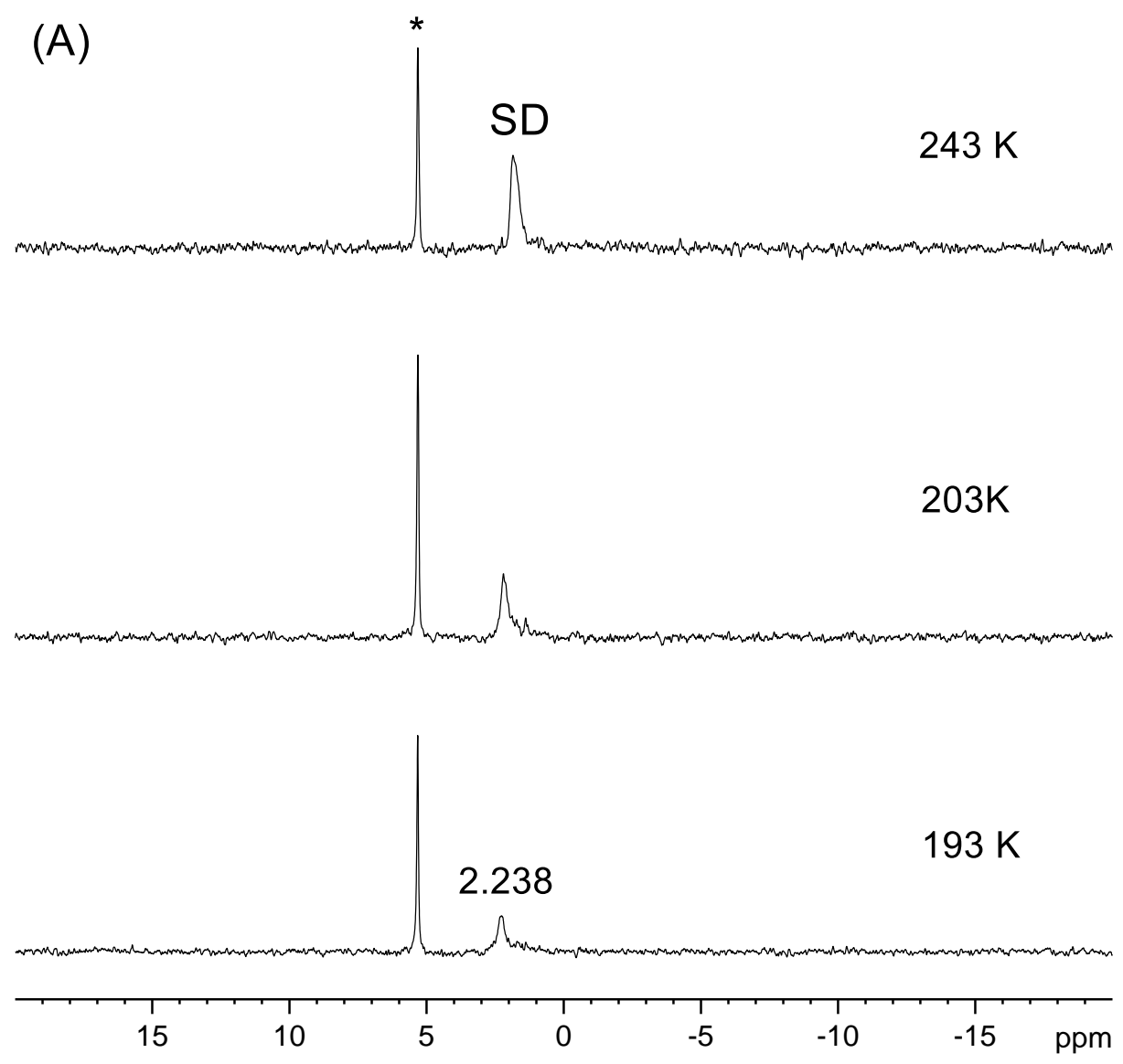

Figure S11. Variable-temperature (A) ${ }^{2} \mathrm{D}$ and (B) ${ }^{1} \mathrm{H}$ NMR spectra of in-situ generated $\mathbf{2} \boldsymbol{\mu H S D}$ * in $\mathrm{CH}_{2} \mathrm{Cl}_{2}$ solution. The solvent signal is indicated as an asterisk. The numbers indicate the chemical shifts of selected peaks. In the ${ }^{1} \mathrm{H}-\mathrm{NMR}$ spectra, the peak of $-12.87 \mathrm{ppm}$ is assigned to the other isomer of $\mathbf{2} \boldsymbol{\mu} \mathbf{H S D}^{*}$. $\mathbf{2} \boldsymbol{\mu} \mathbf{H S D}^{*}$ and $\mathbf{2} \boldsymbol{\mu} \mathbf{H S D}^{\mathrm{in} *}$, are two possible isomeric species, depending on the location of S-D. 


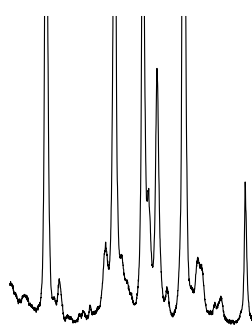

(B)

\section{$243 \mathrm{~K}$}

FeHFe
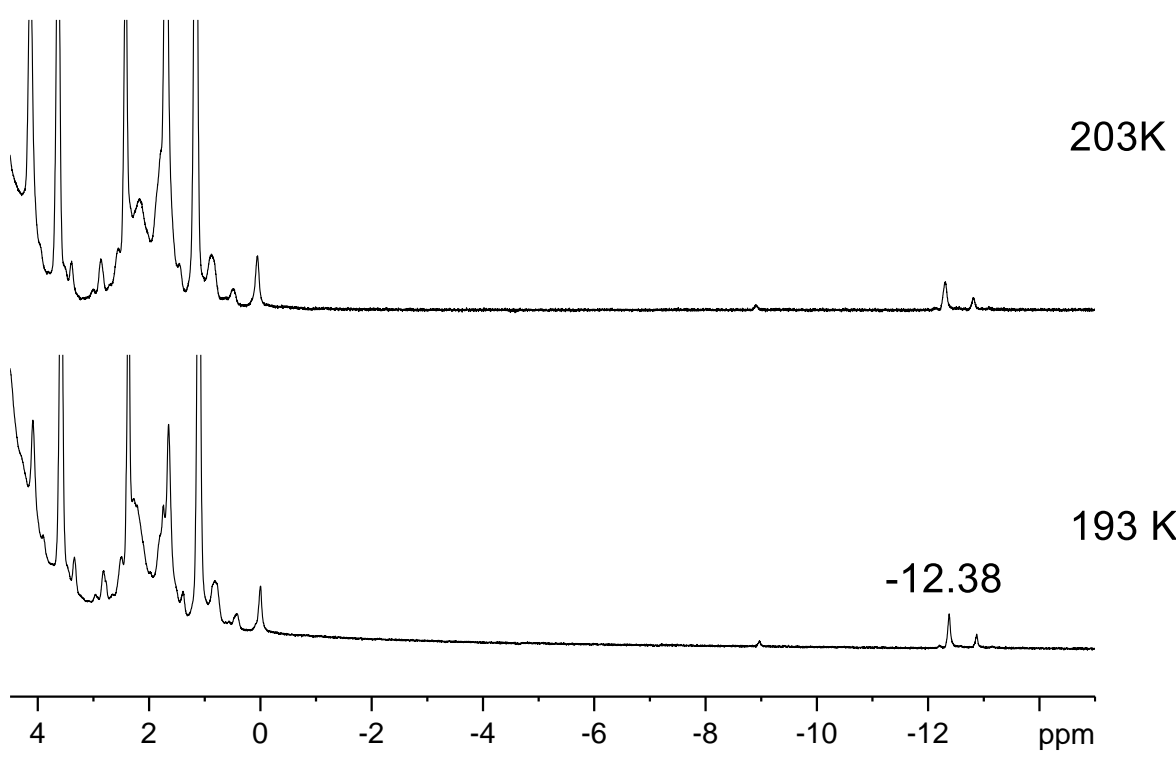

Figure S11. (cont.) 

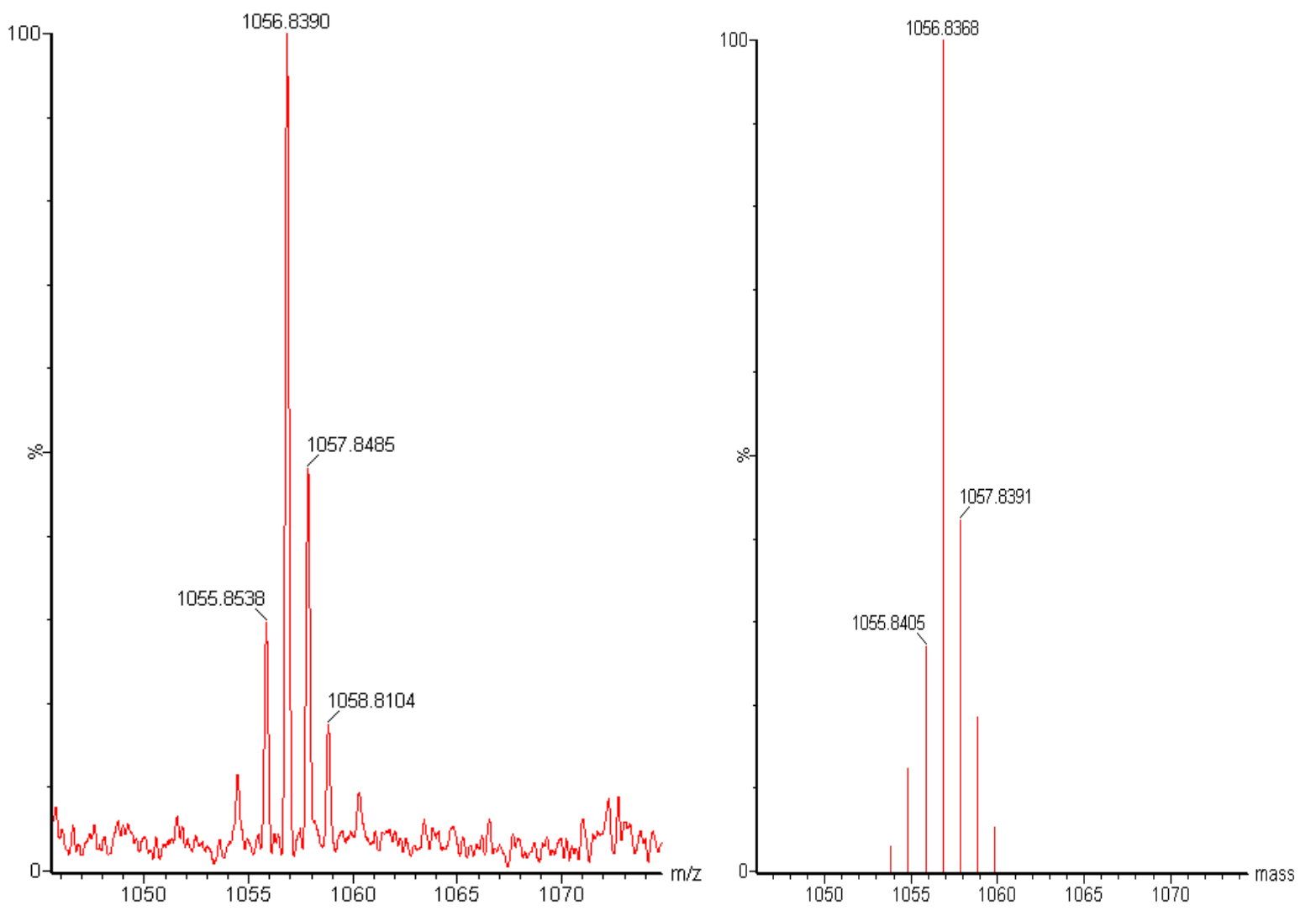

Figure S12. Positive ESI-MS spectra of $\mathbf{2} \boldsymbol{\mu H} \mathbf{H D S D}^{*+}$. Theoretical (right) and experimental (left) isotopic distribution patterns are displayed. 


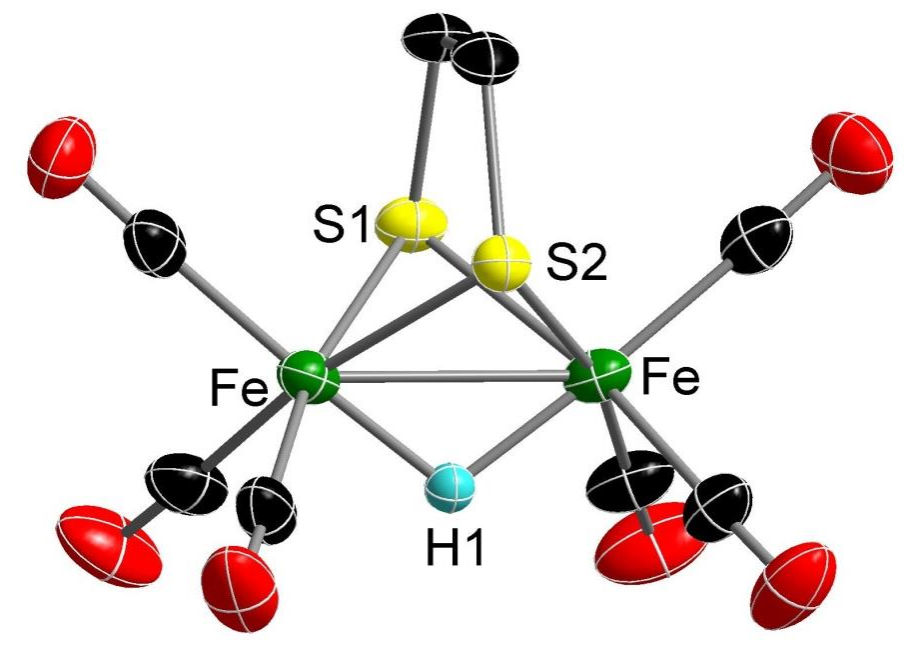

Figure S13. Molecular structure of $[2 \mu \mathrm{H}][\mathrm{OTf}]$, displaying the cationic unit. Only the hydride is shown for clarity. Selected bond length ( $\AA$ ) and angles (deg): $\mathrm{Fe}-\mathrm{Fe}, 2.5625(12) ; \mathrm{Fe}-\mathrm{S}$, ave. 2.2409(12); $\mathrm{Fe}-\mathrm{C}_{\mathrm{CO}, a \mathrm{p}}, 1.802(5) ; \mathrm{Fe}-\mathrm{C}_{\mathrm{CO}, \text { ba, }}$ ave. 1.827(5); $\mathrm{Fe}-\mathrm{H} 1,1.60(4) ; \mathrm{S}-\mathrm{Fe}-\mathrm{S}, 80.33(5)$; $\mathrm{S}-\mathrm{Fe}-\mathrm{Fe}$, ave. 55.13(2); $\mathrm{Fe}-\mathrm{S}-\mathrm{Fe}$, ave. 69.75(5).

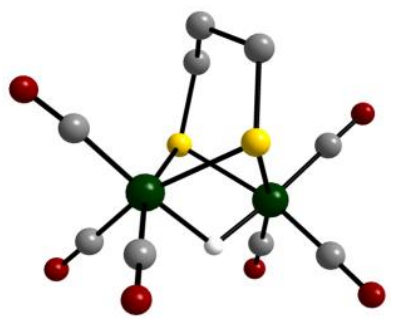

$\mathbf{1} \mu \mathbf{H}$

$\Delta G^{\mathrm{CE}}=0$

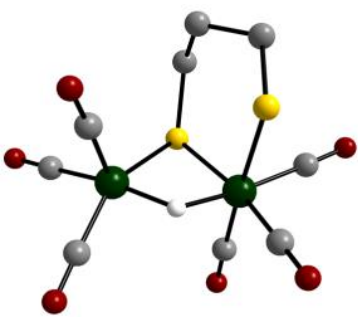

$\mathbf{1} \mu \mathbf{H}^{*}$

$\Delta G^{\mathrm{CE}}=10.09$

Figure S14. Computed structures of the $C E$ species for the pdt analogs. The free energy difference is reported in $\mathrm{kcal} \mathrm{mol}^{-1}$. All hydrogen atoms except for the hydride are omitted for clarity. Color scheme: Fe, dark green; S, yellow; O, red; C, grey and H, white. 


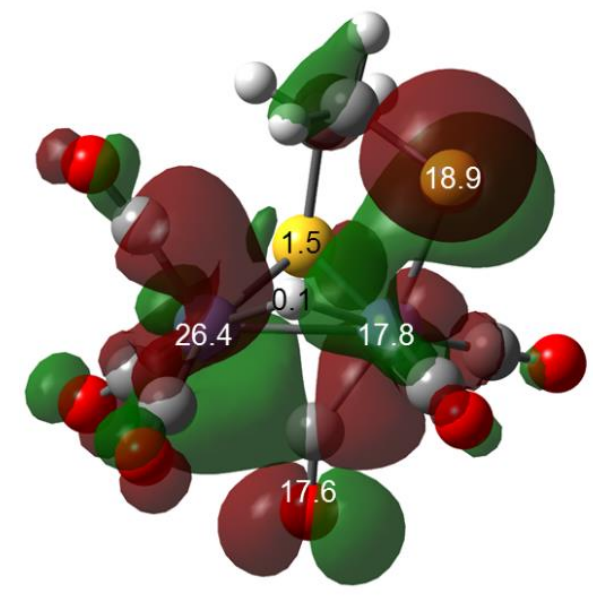

Figure S15. Highest occupied molecular orbital and orbital contribution analysis of $\mathbf{2} \boldsymbol{\mu} \mathbf{H}^{*-}$. The numbers indicate the orbital contribution $(\%)$ for specific atoms: $\mathrm{Fe}^{\mathrm{t}}, 17.8 ; \mathrm{Fe}^{\mathrm{b}}, 26.4 ; \mathrm{S}^{\mathrm{t}}, 18.9 ; \mathrm{S}^{\mathrm{b}}$, $1.5 ; \mathrm{CO}^{\mathrm{b}}, 17.6 ; \mathrm{H}^{\mathrm{b}}, 0.1$.

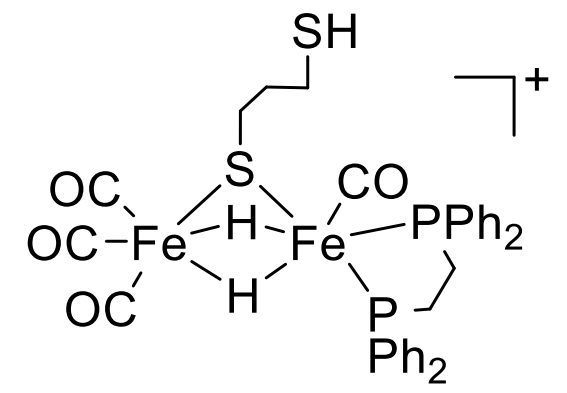

$\left[2-\mathrm{H}_{3}(\mathrm{r})\right]^{+}\left\{\mathrm{Fe}(2 \mathrm{H}) \mathrm{Fe} \_\mathrm{SH}\right\}$

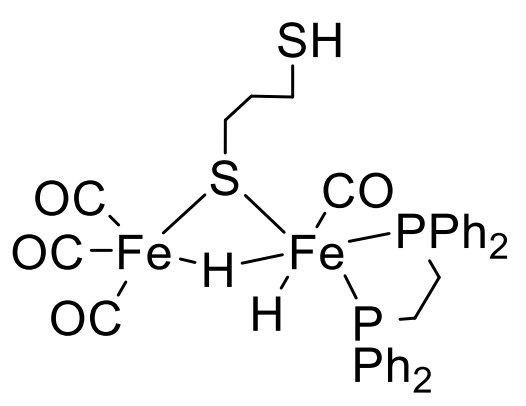

$\left[2-\mathrm{H}_{3}(\mathrm{U})\right]_{\left\{\mathrm{Fe}(2 \mathrm{H}) \mathrm{Fe} \_\mathrm{SH}\right\}}$

Figure S16. Optimized structures of the triprotonated cationic (left) and neutral (right) species. They are the most stable isomers among their peers. The labels for the species are adapted from the literature, Dalton Trans. 2010, 39, 7320. 

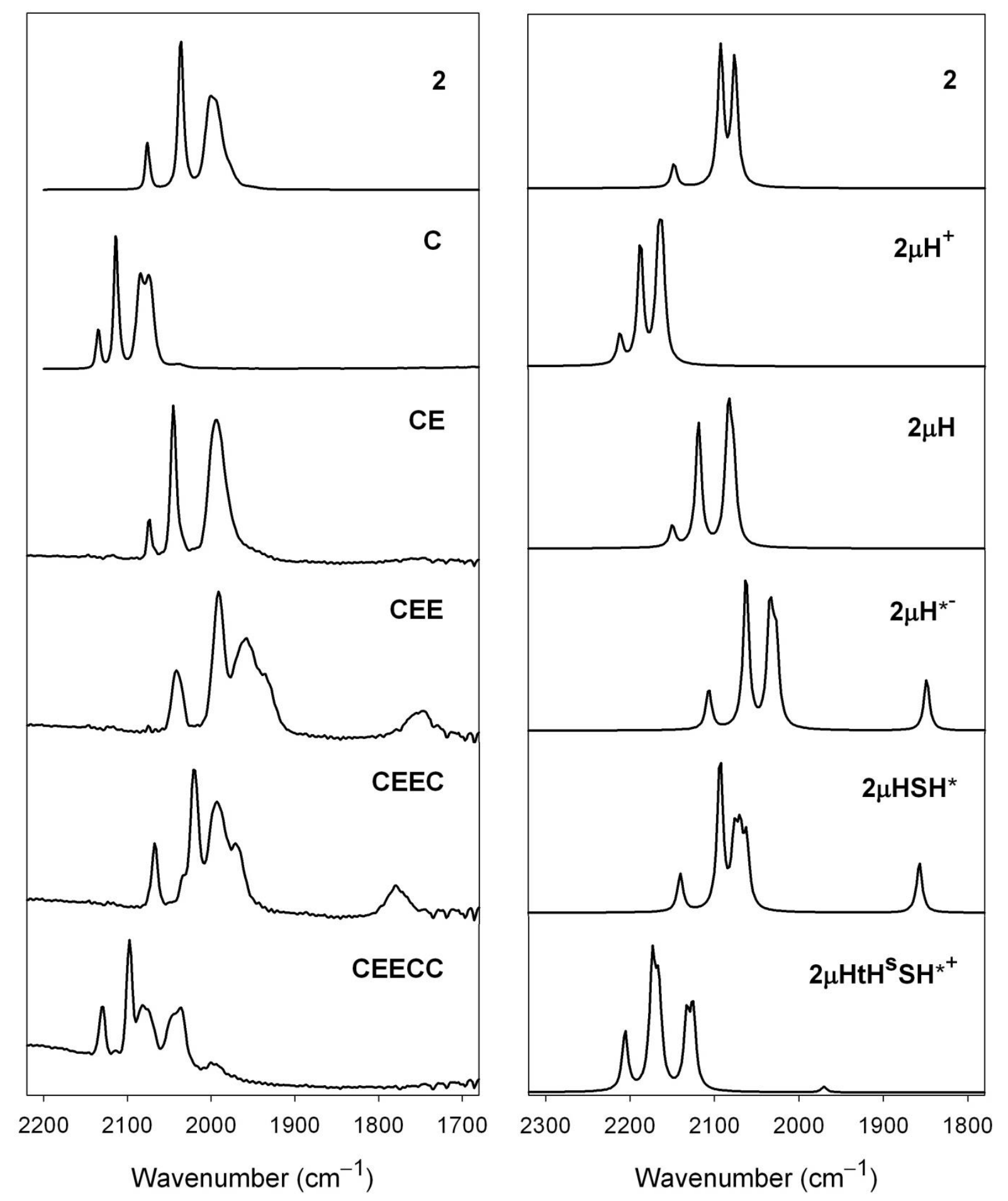

Figure S17. Comparison of experimental (left panel) and calculated (right panel) CO frequencies $\left(\mathrm{cm}^{-1}\right)$ of complex 2 and its reduced/protonated derivatives. 


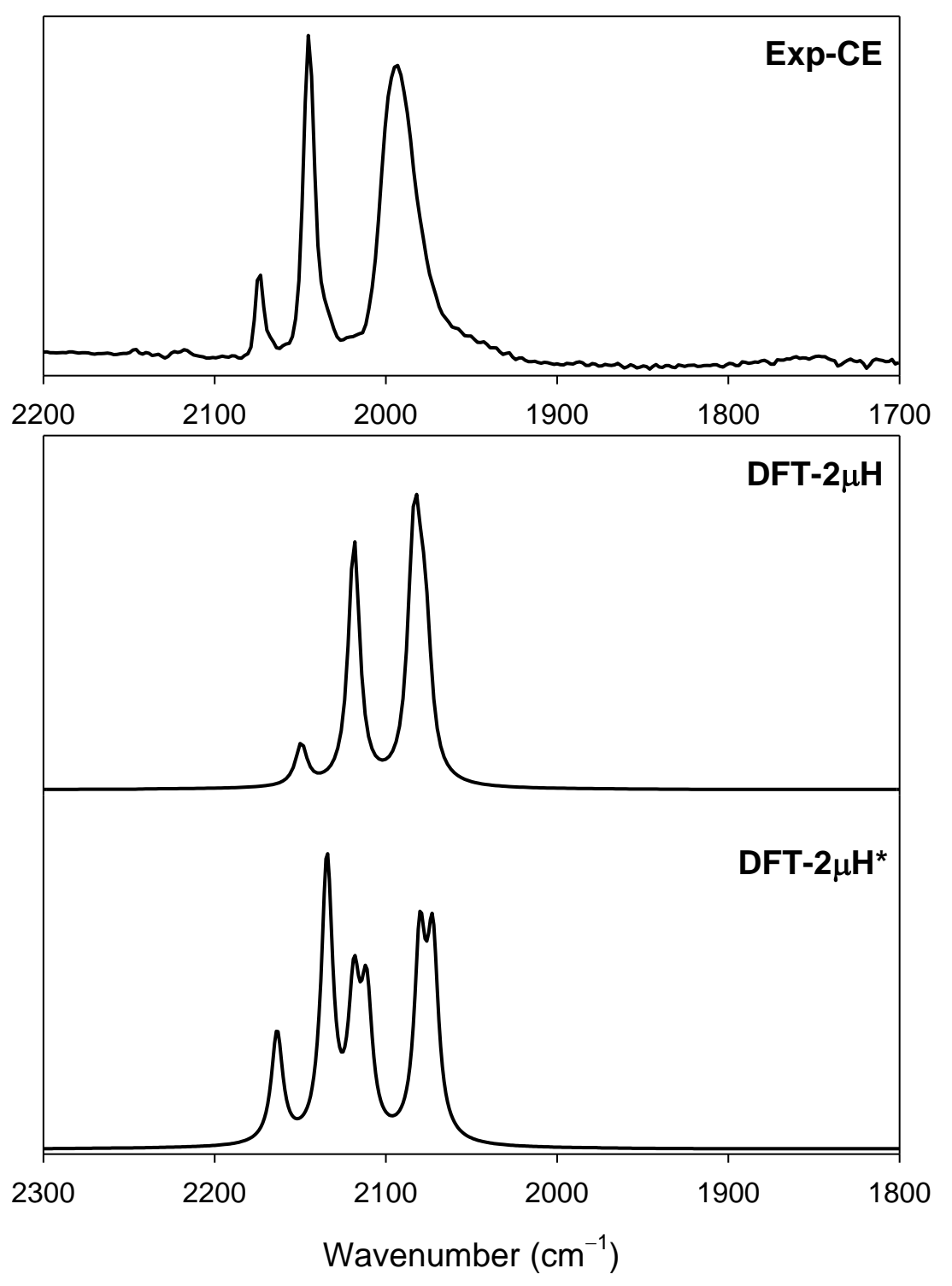

Figure S18. Comparison of experimental and calculated $\mathrm{CO}$ frequencies $\left(\mathrm{cm}^{-1}\right)$ of all reduced/protonated derivatives of complex 2 . 

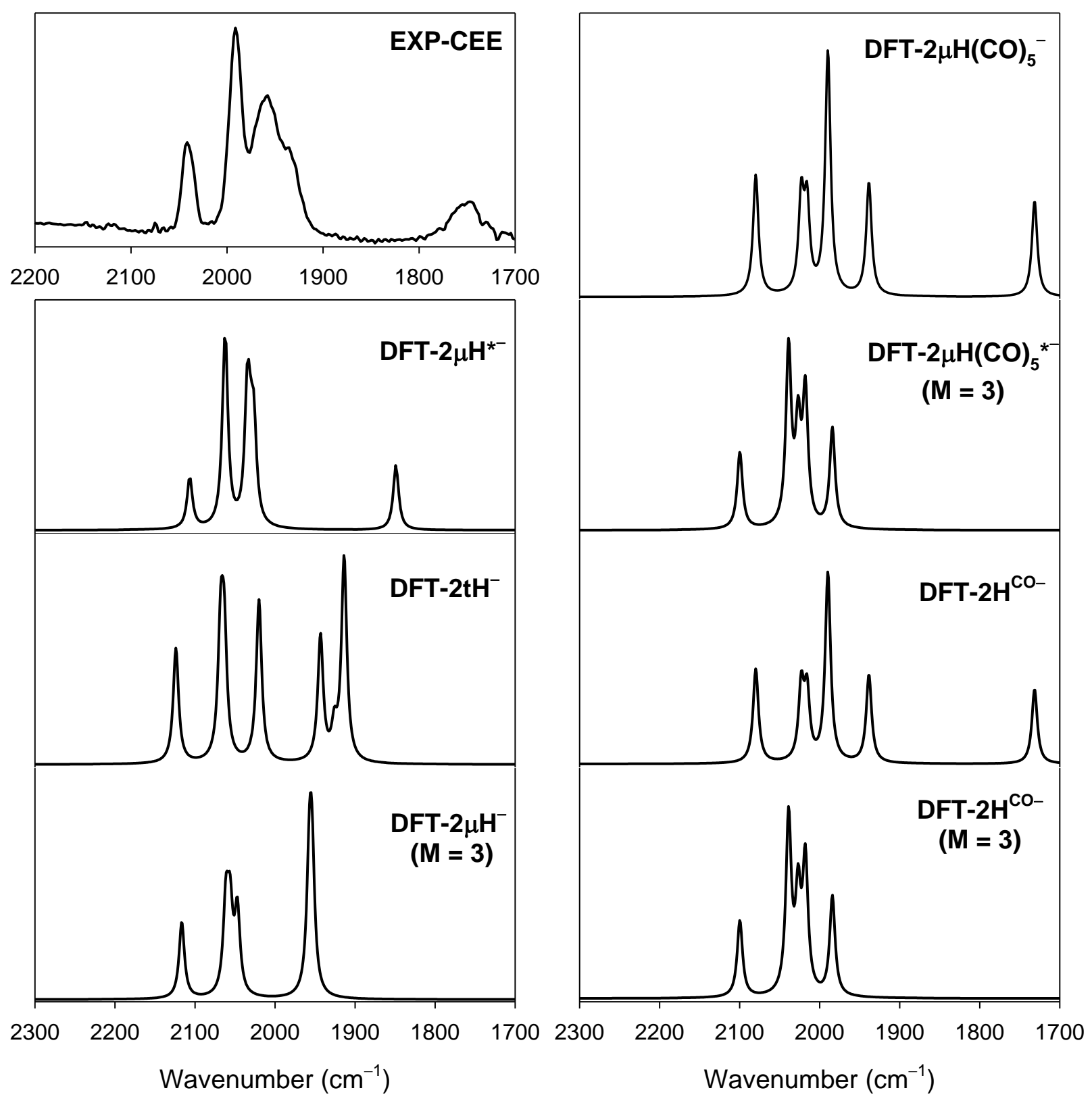

Figure S18. (cont.) 

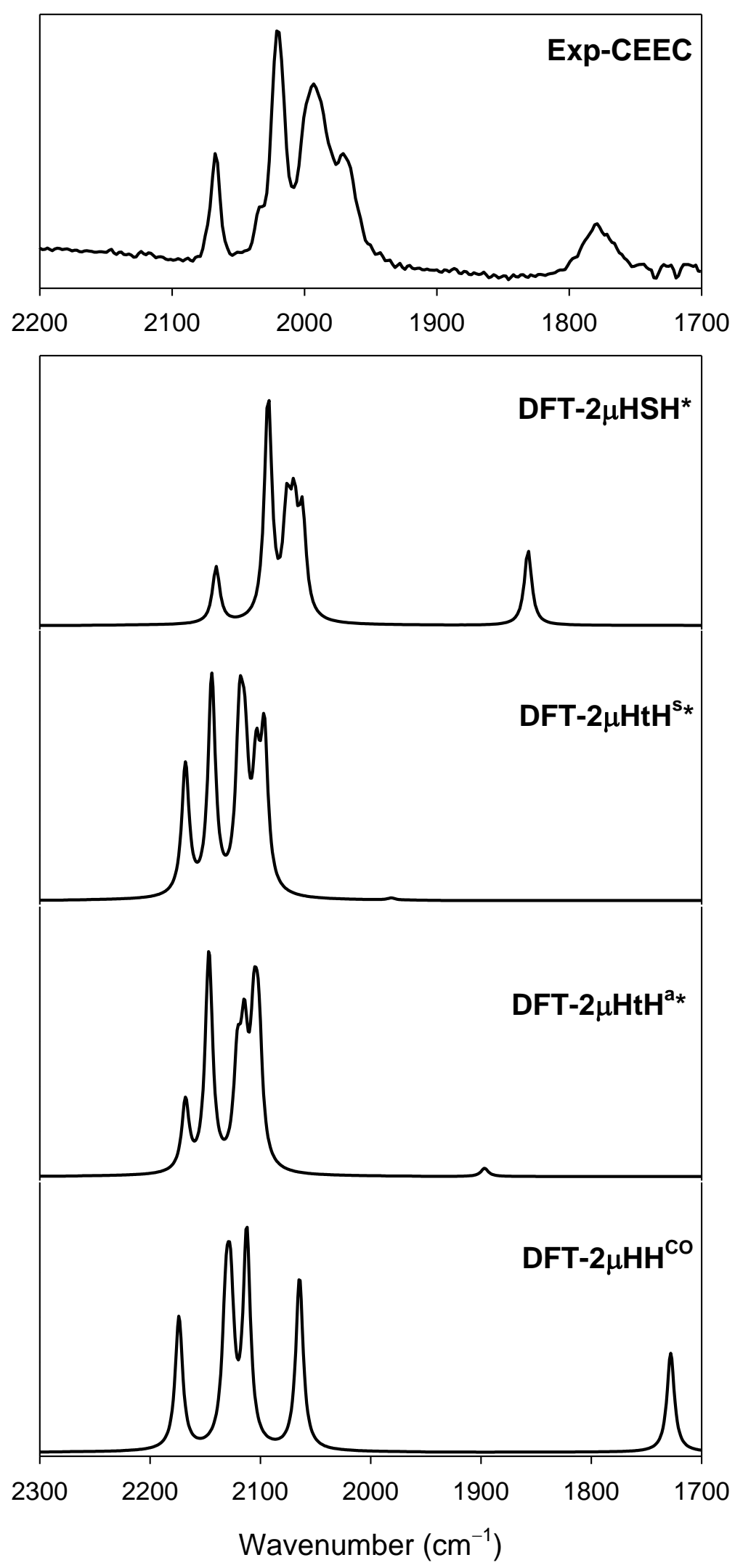

Figure S18. (cont.) 

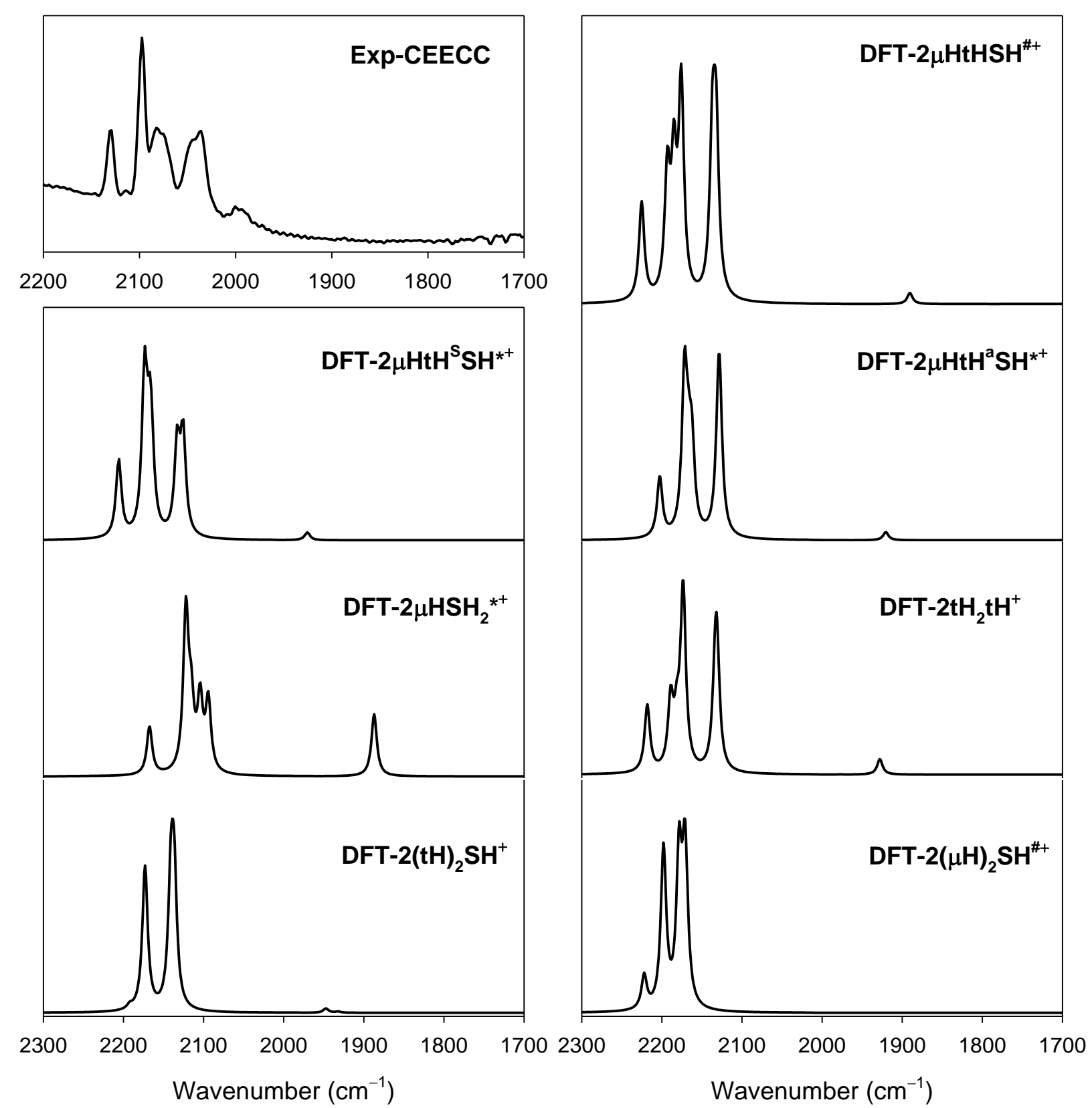

Figure S18. (cont.) 


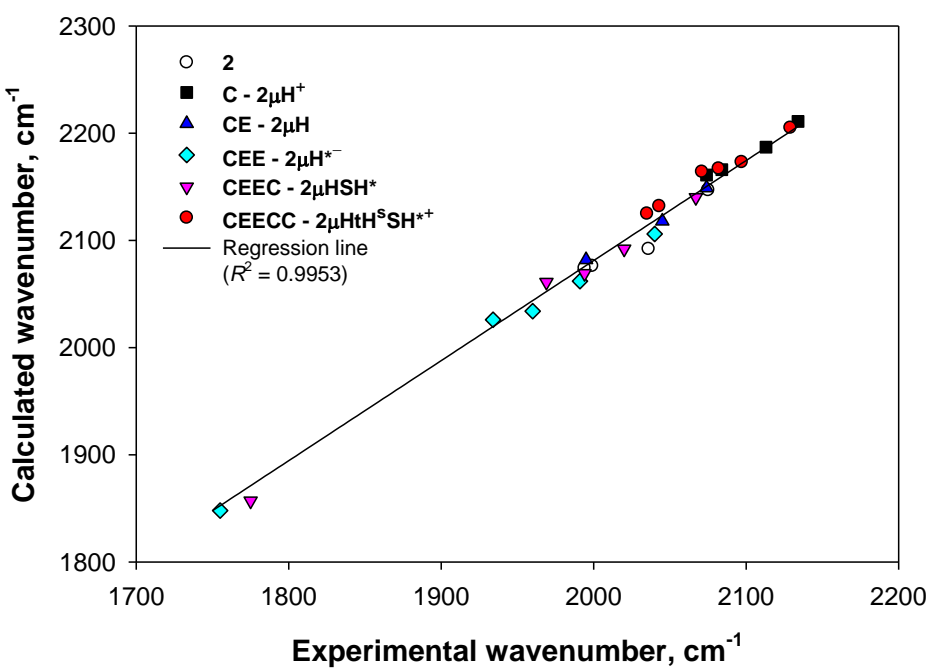

Figure S19. The linear relationship of experimental and calculated CO frequencies $\left(\mathrm{cm}^{-1}\right)$ using B3LYP functional of complex 2 and its reduced/protonated derivatives. The solid line represents the least-squares fit of all data.

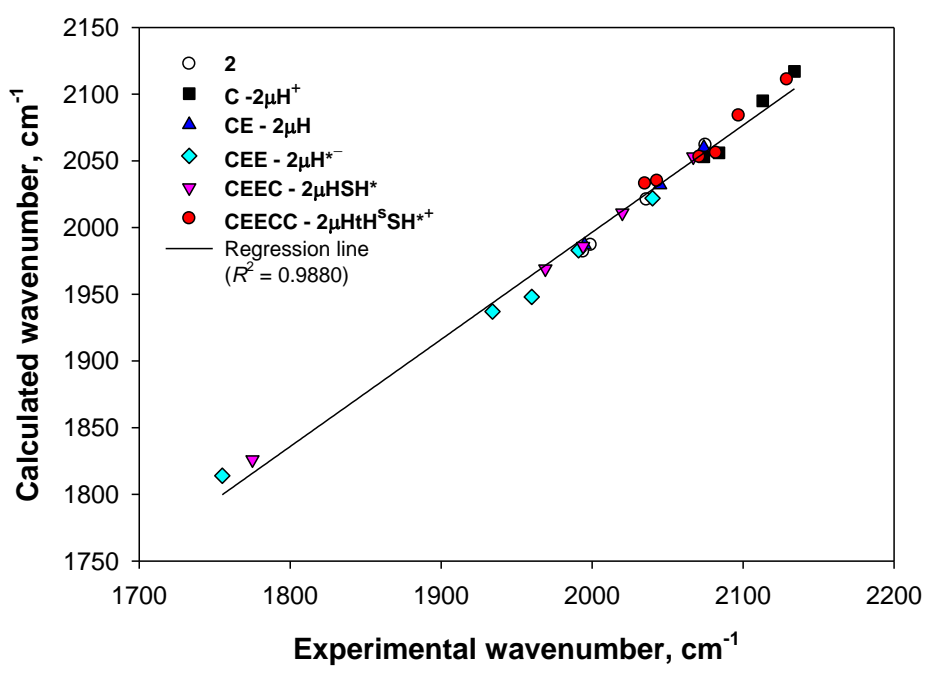

Figure S20. The linear relationship of experimental and calculated CO frequencies $\left(\mathrm{cm}^{-1}\right)$ using BP86 functional of complex 2 and its reduced/protonated derivatives. The solid line represents the least-squares fit of all data. 


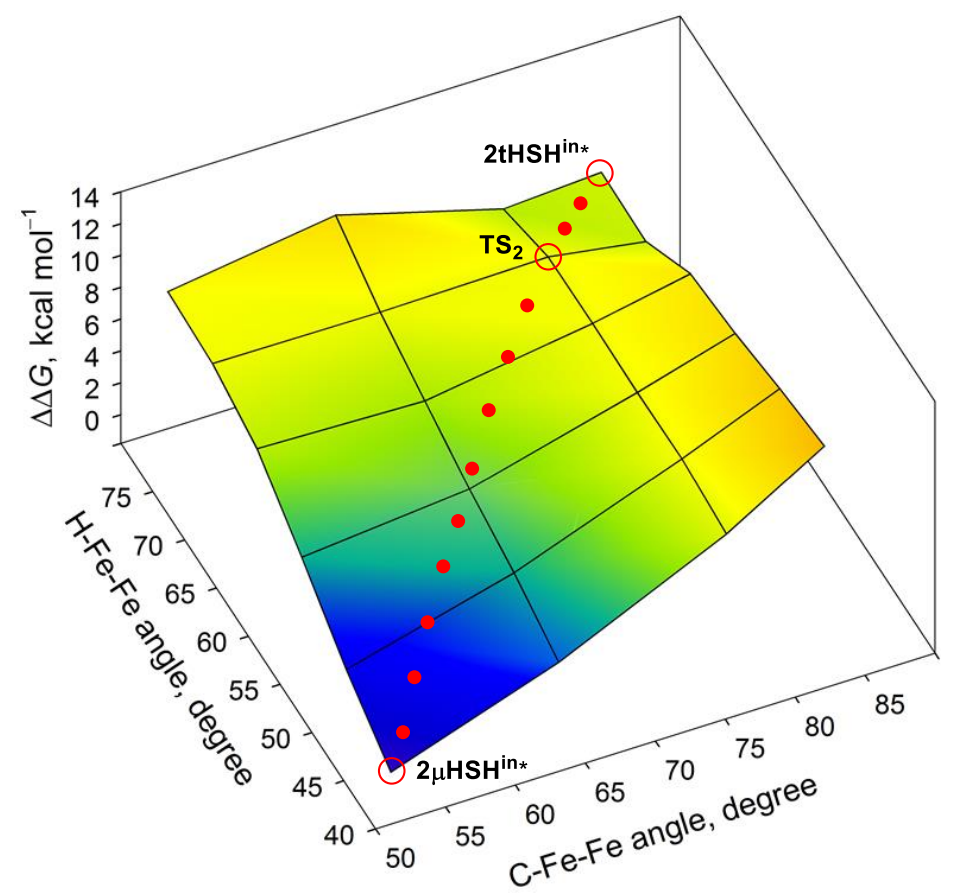

Figure S21. The two dimensional potential energy surface showing the transition from $2 \mu \mathbf{H S H}^{\text {in } *}$ to $\mathbf{2} \mathbf{t H S H}^{\text {in } *}$ corresponds to the changes of $\angle \mathrm{C}-\mathrm{Fe}-\mathrm{Fe}$ and $\angle \mathrm{H}-\mathrm{Fe}-\mathrm{Fe}$. The solid red dots depict the pathway through the transition state $\mathbf{T S}_{2}$.

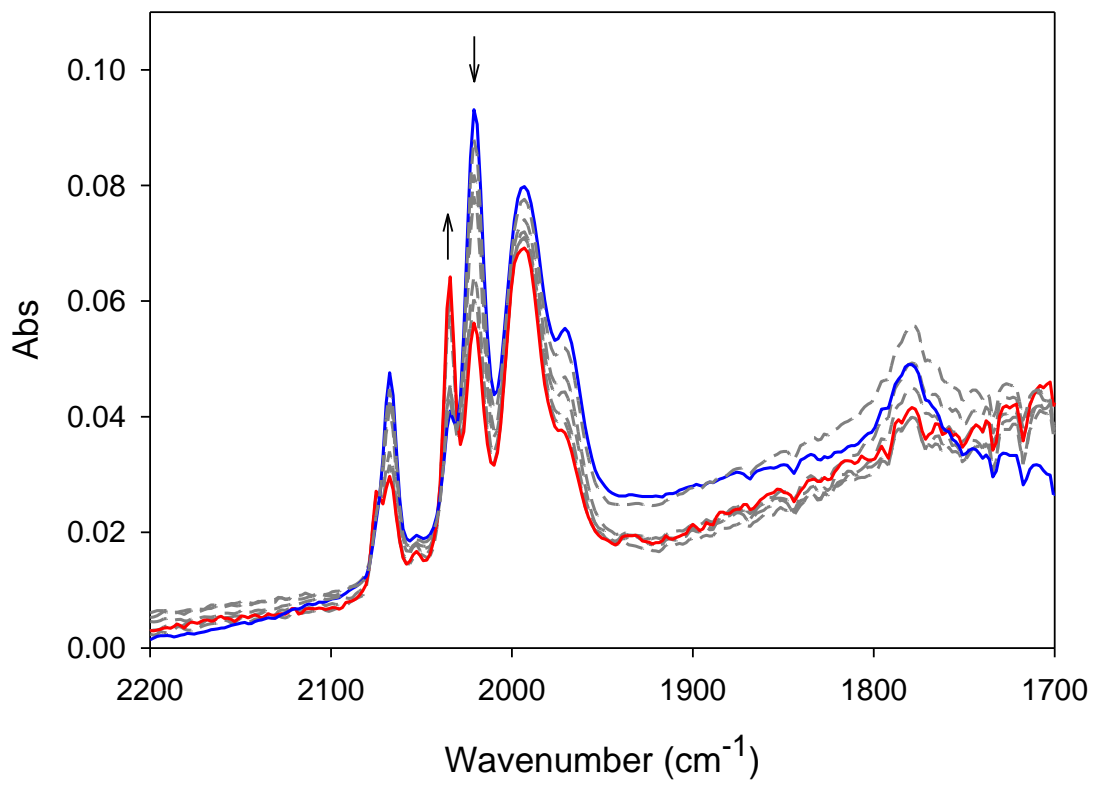

Figure S22. Time-dependent in-situ FTIR spectra of the conversion from $\mathbf{2} \boldsymbol{\mu H S H} *$ (blue line) to 2 (red line) in $\mathrm{CH}_{2} \mathrm{Cl}_{2}$ solution at $213 \mathrm{~K}$. The grey traces show the progressing of the spectral changes. 


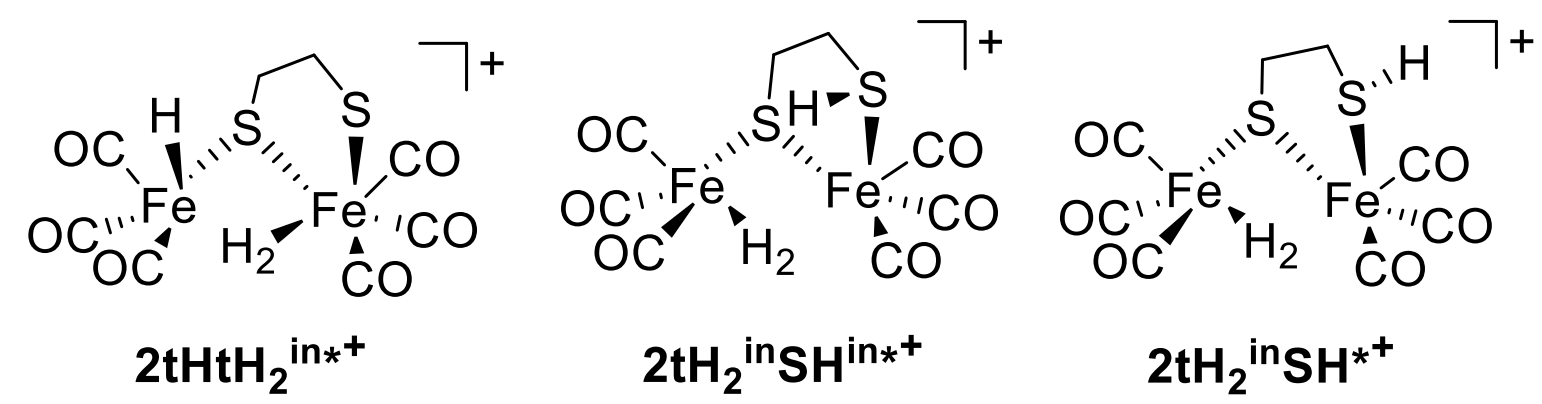

Figure S23. Chemdraw plots of the optimized structures of the intermediate species $\left(\mathbf{2} \mathbf{t H t H} \mathbf{H}^{\text {in } *+}\right.$, $\mathbf{2} \mathbf{t H}_{2}{ }^{\text {in }} \mathbf{S H}^{\text {in }}{ }^{++}, \mathbf{2 t H}_{2}{ }^{\text {ins }} \mathbf{S H}^{*+}$ ) in hydrogen evolution involving $\mathbf{2} \boldsymbol{\mu} \mathbf{H t H}^{\mathrm{s} S \mathbf{S H}^{*+}}$. These species are discussed in main text.

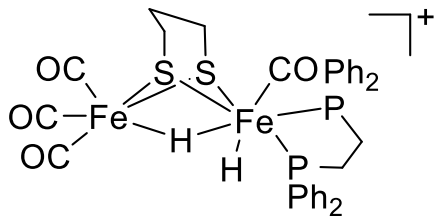

$\left[2-\mathrm{H}_{2}(\mathrm{~b})\right]^{+}\{\mathrm{Fe}(2 \mathrm{H}) \mathrm{Fe}\}$

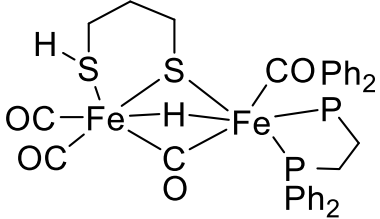

$\left[2-\mathrm{H}_{\mathbf{2}}(\mathrm{h})\right]_{\{\mathrm{Fe}(\mathrm{H}) \mathrm{Fe}} \mathrm{SH}_{\}}$ $\left[(\mu-p d t)(\mu-H)(t-H)\left(\kappa^{2}-d p p e\right) \mathrm{Fe}_{2}(\mathrm{CO})_{4}\right]^{+} \quad\left[\left(\mu, \kappa^{2}-\mathrm{pdtH}\right)(\mu-\mathrm{H})(\mu-\mathrm{CO})\left(\kappa^{2}-\mathrm{dppe}\right) \mathrm{Fe}_{2}(\mathrm{CO})_{3}\right]$

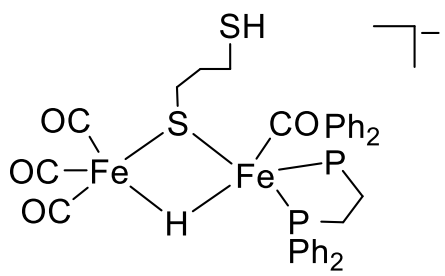

$\left[2-\mathrm{H}_{2}(\mathrm{I})\right]^{-}\left\{\mathrm{Fe}(\mathrm{H}) \mathrm{Fe} \_\mathrm{SH}\right\}$

$\left[\left(\mu-\mathrm{S}\left(\mathrm{CH}_{2}\right)_{3} \mathrm{SH}\right)(\mu-\mathrm{H})\left(\kappa^{2}-\mathrm{dppe}\right) \mathrm{Fe}_{2}(\mathrm{CO})_{4}\right]^{-}$

Figure S24. Optimized structures of the diprotonated species and its two subsequent reduced products. They are the most stable isomers among their peers. The labels for the species are adapted from the literature, Dalton Trans. 2010, 39, 7320. 


\section{Tables}

Table S1. X-ray crystallographic data.

\begin{tabular}{|c|c|c|}
\hline & $\left([\mathbf{1} \boldsymbol{\mu} \mathbf{H}]\left[\mathrm{B}\left(\mathrm{C}_{6} \mathrm{~F}_{5}\right)_{4}\right]\right)_{2} \cdot \mathrm{CH}_{2} \mathrm{Cl}_{2}$ & {$[2 \mu \mathrm{H}]\left[\mathrm{B}\left(\mathrm{C}_{6} \mathrm{~F}_{5}\right)_{4}\right] \cdot \mathrm{CH}_{2} \mathrm{Cl}_{2}$} \\
\hline Empirical formula & $\mathrm{C}_{67} \mathrm{H}_{16} \mathrm{~B}_{2} \mathrm{Cl}_{2} \mathrm{~F}_{40} \mathrm{Fe}_{4} \mathrm{O}_{12} \mathrm{~S}_{4}$ & $\mathrm{C}_{33} \mathrm{H}_{7} \mathrm{BCl}_{2} \mathrm{~F}_{20} \mathrm{Fe}_{2} \mathrm{O}_{6} \mathrm{~S}_{2}$ \\
\hline Formula weight & 2216.96 & 1136.92 \\
\hline$T, \mathrm{~K}$ & $200(2)$ & $200(2)$ \\
\hline Crystal system & Orthorhombic & Monoclinic \\
\hline Space group & $P c a 2_{1}$ & $C c$ \\
\hline$a, \AA$ & $17.7344(5)$ & $22.9134(12)$ \\
\hline$b, \AA$ & $14.2966(4)$ & $12.3868(7)$ \\
\hline$c, \AA$ & $30.6824(9)$ & $17.2161(9)$ \\
\hline$\alpha,^{\circ}$ & 90 & 90 \\
\hline$\beta,{ }^{\circ}$ & 90 & $124.6992(10)$ \\
\hline$\gamma,{ }^{\circ}$ & 90 & 90 \\
\hline$V, \AA^{3}$ & $7779.3(4)$ & $4017.3(4)$ \\
\hline $\mathrm{Z}$ & 4 & 4 \\
\hline$\rho_{\text {calcd }}, \mathrm{Mg} \mathrm{m}^{-3}$ & 1.893 & 1.880 \\
\hline$\mu, \mathrm{mm}^{-1}$ & 1.064 & 1.097 \\
\hline$F(000)$ & 4344 & 2224 \\
\hline Reflections collected & 53971 & 15170 \\
\hline Independent reflections & 17810 & 9032 \\
\hline$R_{\text {int }}$ & 0.0635 & 0.0247 \\
\hline Goodness-of-fit on $\mathrm{F}^{2}$ & 1.036 & 1.042 \\
\hline $\mathrm{R} 1[I>2 \sigma(I)](\text { all data })^{\mathrm{a}}$ & $0.0537(0.0780)$ & $0.0427(0.0473)$ \\
\hline $\mathrm{wR} 2[I>2 \sigma(I)](\text { all data })^{\mathrm{b}}$ & $0.1038(0.1147)$ & $0.1034(0.1063)$ \\
\hline
\end{tabular}

${ }^{\mathrm{a}} \mathrm{R} 1=\left(\Sigma\left\|F_{\mathrm{o}}|-| F_{\mathrm{c}}\right\|\right) /\left(\Sigma\left|F_{\mathrm{o}}\right|\right) \cdot{ }^{\mathrm{b}} \mathrm{wR} 2=\left[\Sigma w\left(F_{\mathrm{o}}{ }^{2}-F_{\mathrm{c}}{ }^{2}\right)^{2} / \Sigma w\left(F_{\mathrm{o}}{ }^{2}\right)^{2}\right]^{1 / 2}$. 
Table S1. (continued) X-ray crystallographic data.

\begin{tabular}{|c|c|c|}
\hline & {$[\mathbf{3} \boldsymbol{\mu} \mathbf{H}]\left[\mathrm{B}\left(\mathrm{C}_{6} \mathrm{~F}_{5}\right)_{4}\right]$} & {$[2 \mu \mathrm{H}]\left[\mathrm{CF}_{3} \mathrm{SO}_{3}\right] \cdot 2\left[\mathrm{CF}_{3} \mathrm{SO}_{3} \mathrm{H}\right]$} \\
\hline Empirical formula & $\mathrm{C}_{36} \mathrm{H}_{5} \mathrm{BF}_{20} \mathrm{Fe}_{2} \mathrm{O}_{6} \mathrm{~S}_{2}$ & $\mathrm{C}_{11} \mathrm{H}_{7} \mathrm{~F}_{9} \mathrm{Fe}_{2} \mathrm{O}_{15} \mathrm{~S}_{5}$ \\
\hline Formula weight & 1100.03 & 822.17 \\
\hline$T, \mathrm{~K}$ & $200(2)$ & $150(2)$ \\
\hline Crystal system & Orthorhombic & Monoclinic \\
\hline Space group & Pbca & $P 2_{1} / m$ \\
\hline$a, \AA$ & $16.0418(6)$ & $6.4147(4)$ \\
\hline$b, \AA$ & $20.9445(8)$ & $18.5996(11)$ \\
\hline$c, \AA$ & $23.0959(10)$ & $11.5206(6)$ \\
\hline$\alpha,^{\circ}$ & 90 & 90 \\
\hline$\beta,^{\circ}$ & 90 & $104.1454(13)$ \\
\hline$\gamma,{ }^{\circ}$ & 90 & 90 \\
\hline$V, \AA^{3}$ & $7759.9(5)$ & $1332.86(13)$ \\
\hline $\mathrm{Z}$ & 8 & 2 \\
\hline$\rho_{\text {calcd }}, \mathrm{Mg} \mathrm{m}^{-3}$ & 1.883 & 2.049 \\
\hline$\mu, \mathrm{mm}^{-1}$ & 0.999 & 1.609 \\
\hline$F(000)$ & 4304 & 812 \\
\hline Reflections collected & 41141 & 8728 \\
\hline Independent reflections & 8911 & 3148 \\
\hline$R_{\text {int }}$ & 0.0689 & 0.0344 \\
\hline Goodness-of-fit on $\mathrm{F}^{2}$ & 1.148 & 1.106 \\
\hline $\mathrm{R} 1[I>2 \sigma(I)](\text { all data })^{\mathrm{a}}$ & $0.0644(0.0902)$ & $0.0577(0.0673)$ \\
\hline $\mathrm{wR} 2[I>2 \sigma(I)](\text { all data })^{\mathrm{b}}$ & $0.1245(0.1346)$ & $0.1301(0.1360)$ \\
\hline
\end{tabular}

${ }^{\mathrm{a}} \mathrm{R} 1=\left(\Sigma\left\|F_{\mathrm{o}}|-| F_{\mathrm{c}}\right\|\right) /\left(\Sigma\left|F_{\mathrm{o}}\right|\right) \cdot{ }^{\mathrm{b}} \mathrm{wR} 2=\left[\Sigma w\left(F_{\mathrm{o}}{ }^{2}-F_{\mathrm{c}}{ }^{2}\right)^{2} / \Sigma w\left(F_{\mathrm{o}}{ }^{2}\right)^{2}\right]^{1 / 2}$. 
Table S2. Selected bond distances $(\AA)$ and angles $\left(^{\circ}\right)$ of the crystallographic data and the DFT geometry-optimized models for $\mathbf{1} \boldsymbol{\mu} \mathbf{H}^{+}, \mathbf{2} \boldsymbol{\mu} \mathbf{H}^{+}$and $\mathbf{3} \boldsymbol{\mu} \mathbf{H}^{+}$.

\begin{tabular}{lcccccc}
\hline & \multicolumn{2}{c}{$\mathbf{1} \boldsymbol{\mu} \mathbf{H}^{+}$} & \multicolumn{2}{c}{$\mathbf{2} \boldsymbol{\mu} \mathbf{H}^{+}$} & \multicolumn{2}{c}{$\mathbf{3} \mathbf{H}^{+}$} \\
\cline { 2 - 7 } & calc. & cryst. & calc. & cryst. & calc. & cryst. \\
\hline Fe(1)-Fe(2) & 2.5789 & $2.5641(10)$ & 2.5716 & $2.5477(7)$ & 2.5607 & $2.5438(7)$ \\
Fe-S, ave. & 2.3319 & $2.2601(15)$ & 2.3177 & $2.2461(10)$ & 2.3394 & $2.27(11)$ \\
Fe-Cco,ap, ave. & 1.8134 & $1.8055(6)$ & 1.8140 & $1.819(4)$ & 1.8217 & $1.8145(4)$ \\
Fe-CCO,ba, ave. & 1.8312 & $1.8233(6)$ & 1.8292 & $1.832(4)$ & 1.8311 & $1.8245(5)$ \\
Fe(1)-H(1) & 1.6640 & $1.70(6)$ & 1.6553 & $1.81(4)$ & 1.6497 & $1.63(4)$ \\
Fe(2)-H(1) & 1.6482 & $1.54(6)$ & 1.6552 & $1.59(5)$ & 1.6524 & $1.66(4)$ \\
S-Fe-S & 85.4752 & $84.46(6)$ & 80.9706 & $80.35(4)$ & 80.9627 & $80.865(4)$ \\
S-Fe-Fe & 56.2821 & $55.41(4)$ & 56.3034 & $55.45(3)$ & 56.8462 & $55.94(3)$ \\
Fe-S-Fe & 67.2317 & $69.12(5)$ & 67.3904 & $69.11(3)$ & 66.3378 & $68.15(3)$ \\
\hline
\end{tabular}

\section{Calculated $\mathrm{p} K_{\mathrm{a}}$ values and reduction potentials}

The $\mathrm{p} K_{\mathrm{a}}$ value concerning each chemical process (the $\mathrm{C}$ step) and the reduction potential concerning each redox process (the E step) were estimated by calculation (Tables S3-S5). In the first C step, $\mathbf{2} \boldsymbol{\mu} \mathbf{H}^{+}$is generated from protonation of $\mathbf{2}$ by $\left[\left(\mathrm{Et}_{3} \mathrm{Si}\right)_{2} \mathrm{H}\right]^{+}$. The depleted species of $\left[\left(\mathrm{Et} 3_{3} \mathrm{Si}\right)_{2} \mathrm{H}\right]^{+}$is yet unexplored according to the literatures when it is used as a proton provider. We tentatively proposed the formation of a $\mathrm{Si}$-Si bond from deprotonation. It is noted that $\left[\left(\mathrm{Et}_{3} \mathrm{Si}\right)_{2} \mathrm{H}\right]^{+}$is more acidic than HOTf according to the free energy change as the $\Delta G\left(\mathrm{H}^{+}\right)^{[(\mathrm{Et} 3 \mathrm{Si}) 2 \mathrm{H}]+}$ of $-261.59 \mathrm{kcal} \mathrm{mol}^{-1}$ is more positive than $\Delta G\left(\mathrm{H}^{+}\right)^{\mathrm{HOTf}}$ of $-266.86 \mathrm{kcal} \mathrm{mol}^{-1}$. Based on the assumption above, the protonation reaction concerning $\mathbf{2}$ is calculated and the reaction is slightly thermodynamically disfavored by $6.23 \mathrm{kcal} \mathrm{mol}^{-1}$, which corresponds to $-\Delta \mathrm{p} K_{\mathrm{a}}=4.55$. It indicates that the $\mathrm{p} K_{\mathrm{a}}^{\mathrm{cal}}$ of $\mathbf{2} \boldsymbol{\mu} \mathbf{H}^{+}$with respect to HOTf $\left(\mathrm{p} K_{\mathrm{a}}{ }^{\mathrm{MeCN}}=2.60\right)$ is -5.79 . We also calculate the $\mathrm{p} K_{\mathrm{a}}$ of $2 \boldsymbol{\mu} \mathbf{H}$ in $\mathrm{CH}_{2} \mathrm{Cl}_{2}$ solution with respect to HOTf, being 17.91. It is in accordance with the previous estimated result (17.6) in MeCN solution. ${ }^{1}$ Given a $\mathrm{p} K_{\text {a }}$ change of $15 \sim 21$ for a species upon one-electron oxidation according to the experimental observation, ${ }^{2,3}$ 
the $\mathrm{p} K_{\mathrm{a}}$ of $2 \mu \mathbf{H}^{+}$is roughly estimated to be around $2.91 \sim-3.09$, which is consistent with the $\mathrm{p} K_{\mathrm{a}}^{\mathrm{cal}}$ of $2 \boldsymbol{\mu} \mathbf{H}^{+}$of -5.79 .

HOTf is used to generate $2 \mu \mathbf{H S H}^{*}$ and $\mathbf{2} \boldsymbol{\mu} \mathbf{H t H}^{\mathrm{S}} \mathbf{S H}^{*+}$ in the second and third protonation reactions, respectively. The third $\mathrm{C}$ step requires a special attention since a $\Delta \mathrm{p} K$ a value of 9.21 is obtained. It is overestimated whereas the result is somehow consistent with the experimental observation as excess HOTf is required for the third C step. We have tried to estimate the more reliable $\mathrm{p} K_{\mathrm{a}}$ value concerning $\mathrm{Fe}(\mathrm{tH})$ in $\mathbf{2} \boldsymbol{\mu} \mathbf{H} \mathbf{t H}^{\mathbf{S}} \mathbf{S} \mathbf{H}^{*+}$. Unfortunately, convergence to $\mathbf{2} \boldsymbol{\mu H S H} \mathbf{H S}^{*}$ is achieved instead of a species resembling $2 \boldsymbol{\mu H} \mathbf{H} \mathbf{H}^{S} \mathbf{S H}{ }^{*+}$ without the terminal hydride when it is optimized. It is said that the most stable species upon protonation of $\mathbf{2} \boldsymbol{\mu H S H} *$ is formed via the $\mathrm{CO}$ migration from the bridging to the terminal position. Such structural rotation causes a large deviation on the calculated $\mathrm{p} K_{\mathrm{a}}(\mathrm{Fe}-\mathrm{tH})$ of $\mathbf{2} \boldsymbol{\mu} \mathbf{H t H}^{\mathrm{S}} \mathbf{S H}^{*+}$ as the $\Delta \mathrm{p} K_{\mathrm{a}}$ only reflects the energy difference between the reactants and products.

Table S3. Calculated $\Delta \mathrm{p} K_{\mathrm{a}}$ values and reduction potentials $\left(E^{\circ}\right.$ vs. $\left.\mathrm{Fc}^{+} / \mathrm{Fc}\right)$ with solvation $\left(\mathrm{CH}_{2} \mathrm{Cl}_{2}\right)$ correction of the protonated as well as reduced species of complex 2 .

\begin{tabular}{|c|c|c|c|c|}
\hline Step & Reaction & $\Delta G_{\mathrm{rxn}}$ & $-\Delta \mathrm{p} K_{\mathrm{a}}^{\S}$ & $\begin{array}{c}E^{\mathrm{cal}} \\
\left(\mathrm{V} v s . \mathrm{Fc}^{+} / \mathrm{Fc}\right)\end{array}$ \\
\hline $\mathrm{C}$ & $\mathbf{2}+\mathrm{H}^{+}\left(\mathrm{Et}_{3} \mathrm{Si}\right)_{2} \rightarrow \mathbf{2} \boldsymbol{\mu} \mathbf{H}^{+}+\left(\mathrm{Et}_{3} \mathrm{Si}\right)_{2}$ & 6.23 & $4.55^{\dagger}$ & - \\
\hline $\mathrm{CE}$ & $2 \mu \mathbf{H}^{+}+\mathrm{e}^{-} \rightarrow 2 \mu \mathrm{H}$ & - & - & -1.02 \\
\hline \multirow[t]{2}{*}{ CEE } & $2 \mu \mathrm{H}+\mathrm{e}^{-} \rightarrow 2 \mu \mathrm{H}^{*^{-}}$ & - & - & -2.13 \\
\hline & $2 \mu \mathbf{H}^{*}+\mathrm{e}^{-} \rightarrow 2 \mu \mathbf{H}^{*^{-}}$ & - & - & -1.69 \\
\hline CEEC & 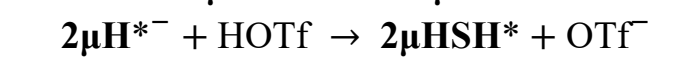 & -15.91 & -11.61 & - \\
\hline \multirow[t]{3}{*}{ CEECC } & $2 \mu \mathbf{H S H}^{*}+\mathrm{HOTf} \rightarrow \mathbf{2 \mu} \mathbf{H t H}^{\mathrm{S}} \mathrm{SH}^{*^{+}}+\mathrm{OTf}^{-}$ & 12.62 & $9.21^{\dagger}$ & - \\
\hline & $\mathbf{2}^{-}+\mathrm{HOTf} \rightarrow \mathbf{2} \boldsymbol{\mu} \mathbf{H}+\mathrm{OTf}^{-}$ & -20.97 & -15.31 & \\
\hline & $\mathrm{HOTf}+\left(\mathrm{Et}_{3} \mathrm{Si}\right)_{2} \rightarrow \mathrm{H}^{+}\left(\mathrm{Et}_{3} \mathrm{Si}\right)_{2}+\mathrm{OTf}^{-}$ & 5.26 & 3.84 & \\
\hline
\end{tabular}

\footnotetext{
${ }^{\S} \Delta \mathrm{p} K_{\mathrm{a}}=-\Delta G_{\mathrm{rxn}} / 1.37$. ${ }^{\dagger}$ The calculated value does not represent the accurate $\mathrm{p} K_{\mathrm{a}}$ value. See the text in the supporting information.
} 
Table S4. Estimated $\mathrm{p} K_{\mathrm{a}}$ values from the calculation with solvation $\left(\mathrm{CH}_{2} \mathrm{Cl}_{2}\right)$ correction of the protonated as well as reduced species of complex 2.

\begin{tabular}{|c|c|}
\hline Reaction & $\mathrm{p} K_{\mathrm{a}}^{\text {calई }}$ \\
\hline $\mathrm{HOTf} \rightarrow \mathrm{OTf}^{-}+\mathrm{H}^{+}$ & $2.60^{\diamond}$ \\
\hline $\mathrm{H}^{+}\left(\mathrm{Et}_{3} \mathrm{Si}\right)_{2} \rightarrow\left(\mathrm{Et}_{3} \mathrm{Si}\right)_{2}+\mathrm{H}^{+}$ & -1.24 \\
\hline $2 \mu \mathbf{H}^{+} \rightarrow \mathbf{2}+\mathrm{H}^{+}$ & $-5.79^{\dagger}$ \\
\hline $2 \mu \mathbf{H} \rightarrow 2^{-}+\mathrm{H}^{+}$ & $17.91^{\ddagger}$ \\
\hline $2 \mu \mathrm{HSH}^{*} \rightarrow 2 \mu \mathrm{H}^{*^{-}}+\mathrm{H}^{+}$ & 14.21 \\
\hline $2 \mu \mathrm{HtH}^{\mathrm{S}} \mathrm{SH}^{*^{+}} \rightarrow 2 \mu \mathrm{HSH}^{*}+\mathrm{H}^{+}$ & $-6.61^{\dagger}$ \\
\hline
\end{tabular}

${ }^{\S}$ The estimated value with respect to HOTf. ${ }^{\diamond}$ in MeCN solution. It is used as a reference. ${ }^{\dagger}$ This is related to the EC route. The $\mathrm{p} K_{\mathrm{a}}^{\mathrm{cal}}$ is estimated for comparison. See the text in the supporting information. The calculated value does not represent the accurate $\mathrm{p} K_{\mathrm{a}}$ value. See the text in the supporting information.

The reduction potential of first $\mathrm{E}$ step is calculated to be $-1.02 \mathrm{~V} v s$. $\mathrm{Fc}^{+} / \mathrm{Fc}$. The difference of $\sim 300 \mathrm{mV}$ from the experimental value is within the acceptable deviation for calculation. ${ }^{4,5}$ The reduction of $2 \boldsymbol{\mu H}$ to $2 \mu \mathbf{H}^{*^{-}}$is calculated to occur at a much negative potential of $-2.13 \mathrm{~V}$. It is inconsistent with the experimental observation that $\mathrm{Cp}_{2} \mathrm{Co}\left(E_{1 / 2}=-1.33 \mathrm{~V}\right)$ is capable of the second reduction. It has been suggested in the literature ${ }^{6}$ that the first reduction of $\mathbf{2}$ yields the one-electron reduced species $\mathbf{2}^{-} \cdot \mathbf{2}^{-}$kinetically tautomerizes to species $\mathbf{2}^{@-}$ (denoted as $\mathbf{A}^{-} \rightarrow \mathbf{B}^{-}$in the literature) which accepts the second electron at a potential more positive than that for the $\mathbf{2}^{-} / \mathbf{2}^{2-}$ pair. Herein, we assume that subsequent tautomerization of $\mathbf{2} \boldsymbol{\mu H}$ to $\mathbf{2} \boldsymbol{\mu} \mathbf{H}^{*}$ occurs after the first reduction. By using $2 \mu \mathbf{H}^{*}$, the calculated potential for the second reduction is $-1.69 \mathrm{~V}$, which is close to the $\mathrm{Cp}_{2} \mathrm{Co}^{+/ 0}$ redox couple.

The theoretical reduction potentials of the reactions and $\mathrm{Fc}^{+} / \mathrm{Fc}$ couple are estimated from the relationship and equations in the following table where $n$ is the number of electron involved, and $F$ the Faraday constant. ${ }^{7}$ The calibrated potentials was obtained with $E\left(\mathrm{~A} / \mathrm{A}^{-}\right)=E^{\circ}\left(\mathrm{A} / \mathrm{A}^{-}\right)-E^{\circ}\left(\mathrm{Fc}^{+} / \mathrm{Fc}\right)$. 
Table S5. Thermodynamic relationships and equations for theoretical estimation of the reduction potential.

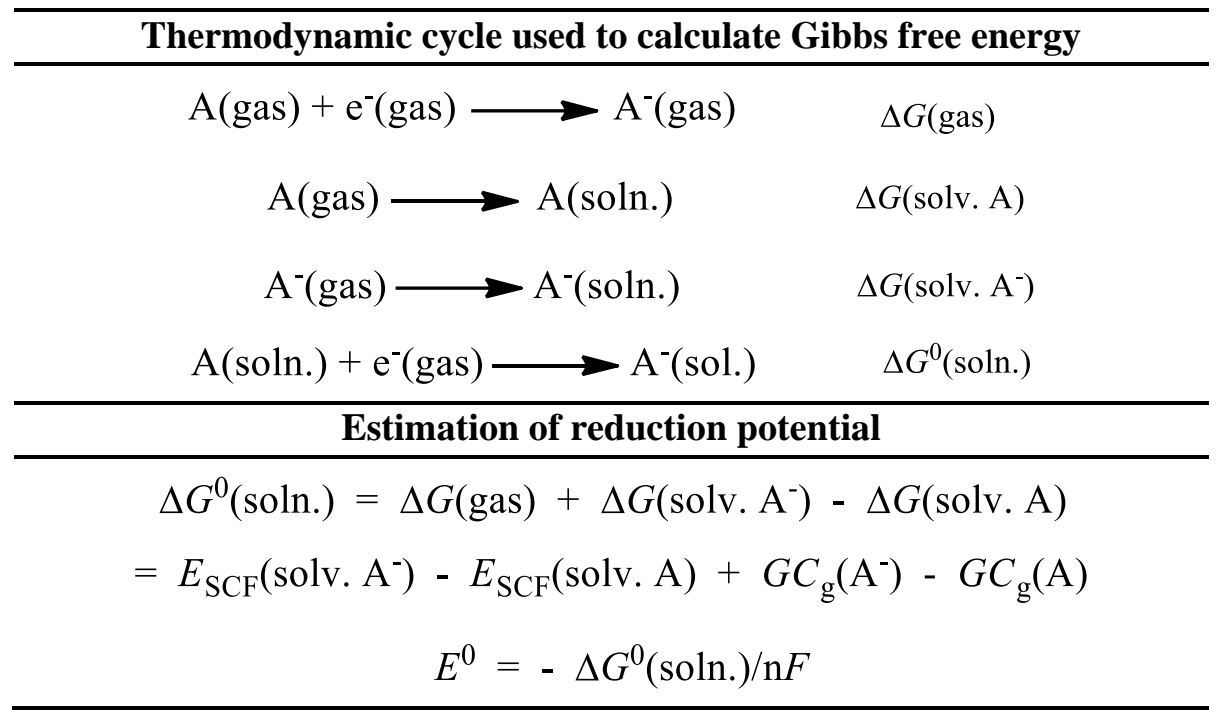

\begin{tabular}{ccc}
\hline Species & $E_{\mathrm{SCF}}($ hartree $)$ & $G C_{\mathrm{g}}$ (hartree) \\
\hline $\mathbf{2} \boldsymbol{\mu} \mathbf{H}^{+}$ & -1802.445722 & 0.0725 \\
$\mathbf{2} \boldsymbol{\mu} \mathbf{H}$ & -1802.599671 & 0.064295 \\
$\mathbf{2} \boldsymbol{\mu} \mathbf{H}^{*}$ & -1802.586789 & 0.067293 \\
$\mathbf{2} \boldsymbol{\mu} \mathbf{H}^{*-}$ & -1802.726334 & 0.069573 \\
$\mathrm{Fc}^{+}$ & -510.3287144 & 0.134865 \\
$\mathrm{Fc}$ & -510.5297447 & 0.13616 \\
\hline
\end{tabular}


Table S6. Summary of solvent corrected vCO IR spectra $\left(10^{-3}\right.$ intensity in parentheses) for proposed intermediates related to complex $\mathbf{2}$ and imaginary frequencies for the transition states.

\begin{tabular}{|c|c|c|}
\hline State & Species & Computed IR spectra, $\mathrm{cm}^{-1}$ \\
\hline & 2 & 2147 (0.68), $2092(3.97), 2076(2.08), 2074$ (1.70) \\
\hline $\mathrm{C}$ & $2 \mu \mathbf{H}^{+}$ & $2211(0.45), 2187(1.84), 2166(1.45), 2161$ (1.22) \\
\hline \multirow[t]{2}{*}{$\mathrm{CE}$} & $2 \mu \mathrm{H}$ & $\begin{array}{c}2149(0.44), 2118(2.61), 2084(1.35), 2082 \text { (1.47), } \\
2078(0.81), 2075(0.76)\end{array}$ \\
\hline & $2 \mu \mathbf{H}^{*}$ & $\begin{array}{c}2163(0.71), 2134(1.81), 2118(0.90), 2111(0.87) \text {, } \\
2080(1.22), 2072(1.20)\end{array}$ \\
\hline \multirow[t]{5}{*}{ CEE } & $2 \mathbf{t} \mathbf{H}^{-}$ & $\begin{array}{c}2123(1.18), 2067(1.28), 2063(1.20), 2020(1.67), \\
1943(1.29), 1925(0.32), 1913(2.10)\end{array}$ \\
\hline & $2 \mu \mathbf{H}^{*-}$ & $\begin{array}{c}2106(0.81), 2062(3.07), 2034(2.00), 2030(0.65), \\
2026(1.41), 1848(1.02)\end{array}$ \\
\hline & $\mathbf{2} \boldsymbol{\mu} \mathbf{H}^{-}(\mathrm{M}=3)$ & $\begin{array}{c}2116 \text { (1.21), } 2061 \text { (1.41), } 2056 \text { (1.25), } 2047 \text { (1.32), } \\
1957(1.93), 1953(1.88)\end{array}$ \\
\hline & $2 \mu \mathrm{H}(\mathrm{CO})_{5}^{-}$ & $\begin{array}{c}2079 \text { (1.76), } 2022(1.38), 2015(1.26), 1989 \text { (3.55), } \\
1938(1.65), 1731(1.40)\end{array}$ \\
\hline & $2 \mu \mathrm{H}(\mathrm{CO})_{5} *^{-}(\mathrm{M}=3)$ & $\begin{array}{c}2099 \text { (1.05), } 2038(2.42), 2026(1.29), 2017 \text { (1.79), } \\
1984(1.36)\end{array}$ \\
\hline \multirow[t]{4}{*}{ CEEC } & $2 \mu \mathrm{HSH}^{*}$ & $\begin{array}{c}2140(0.71), 2092(2.82), 2075(1.24), 2069 \text { (1.17), } \\
2061(1.20), 1857(0.95)\end{array}$ \\
\hline & $2 \mu \mathrm{HtH}^{*}$ & $\begin{array}{c}2167(0.95), 2144(1.57), 2118(1.12), 2114(0.83) \text {, } \\
2103(0.80), 2096(1.07)\end{array}$ \\
\hline & $2 \mu \mathrm{HH} \mathrm{H}^{\mathrm{CO}}$ & $\begin{array}{c}2173(0.99), 2131(0.91), 2127(0.94), 2112(1.57) \text {, } \\
2064(1.28), 1727(0.73)\end{array}$ \\
\hline & $2 \mathrm{tHSH}^{\text {in } *}$ & $\begin{array}{c}2134(0.8), 2076(3.56), 2062(2.47), 2057(1.23) \\
2052(0.45), 2038(0.23)\end{array}$ \\
\hline \multirow[t]{14}{*}{ CEECC } & $2 \mu \mathrm{HtH}^{\mathrm{SSH}}{ }^{*+}$ & $\begin{array}{c}2205(0.74), 2173(1.50), 2167(0.73), 2164(0.55), \\
2132(0.85), 2125(0.96)\end{array}$ \\
\hline & $2 \mu \mathrm{HSH}_{2} *+$ & $\begin{array}{c}2167(0.76), 2122(2.51), 2115(0.93), 2104(1.10), \\
2094(1.11), 1887(0.98)\end{array}$ \\
\hline & $2(\mathbf{t H})_{2} \mathrm{SH}^{+}$ & $2173(2.11), 2140(1.72), 2136(1.72)$ \\
\hline & $2 \mu \mathrm{HtHSH}^{\#+}$ & $\begin{array}{c}2225(0.57), 2193(0.70), 2184(0.74), 2175(1.23), \\
2136(0.90), 2132(0.92)\end{array}$ \\
\hline & $2 \mu \mathrm{HtH}^{\mathrm{a}} \mathrm{SH}^{*+}$ & $\begin{array}{c}2202(0.59), 2171(1.53), 2166(0.52), 2162(0.75), \\
2129(0.96), 2127(0.91)\end{array}$ \\
\hline & $2 \mathbf{t H}_{2} \mathbf{t H}^{+}$ & $\begin{array}{c}2218(0.67), 2188(0.68), 2181(0.42), 2173(1.82), \\
2133(0.85), 2130(0.89)\end{array}$ \\
\hline & $2 \mu \mathbf{H}_{2} \mathrm{SH}^{\#+}$ & $\begin{array}{c}2222(0.32), 2197(1.52), 2178(1.40), 2171(1.24), \\
2169(0.30)\end{array}$ \\
\hline & Transitions & Imaginary frequency, $\mathrm{cm}^{-1}$ \\
\hline & $\mathbf{T S}_{\mathbf{1}}$ & -512.38 \\
\hline & $\mathbf{T S}_{\mathbf{2}}$ & -23.75 \\
\hline & $\mathbf{T S}_{3}$ & -668.19 \\
\hline & $\mathbf{T S}_{\mathbf{2}}$ & -292.34 \\
\hline & $\mathbf{T S}_{\mathbf{3}}$, & -319.49 \\
\hline & $\mathbf{T S}_{4}$ & -292.53 \\
\hline
\end{tabular}


$\mathbf{T S}_{5}$

TS $_{6}$

$\mathbf{T S}_{7}$

TS 8

TS9

TS $_{10}$

TS $_{11}$

$\mathbf{T S}_{12}$

$\mathbf{T S}_{13}$

$\mathrm{TS}_{14}$

$\mathrm{TS}_{15}$
$-59.85$

$-472.44$

$-115.26$

$-837.91$

$-346.77$

$-331.88$

$-942.93$

$-1019.80$

$-128.91$

$-361.21$

$-54.34$ 
Table S7. Summary of solvent corrected free energy for all computed species and transition states.

\begin{tabular}{|c|c|c|}
\hline State & Species & Solvent corrected free energy, hartree \\
\hline & 1 & -1841.246506 \\
\hline $\mathrm{C}$ & $1 \mu \mathrm{H}^{+}$ & -1841.654855 \\
\hline $\mathrm{CE}$ & $1 \mu \mathrm{H}^{1}$ & $\begin{array}{l}-1841.816502 \\
-184180043\end{array}$ \\
\hline \multirow{3}{*}{ CEE } & $1 \mu \mathrm{H}^{-}$ & $\begin{array}{l}-1041.00045 \\
-1841.917873\end{array}$ \\
\hline & $\mathbf{1} \boldsymbol{\mu} \mathbf{H}^{-}(\mathrm{M}=3)$ & -1841.92819 \\
\hline & $1 \mu \mathrm{H}^{*^{-}}$ & -1841.934632 \\
\hline \multirow[t]{3}{*}{ State } & Species & Solvent corrected free energy, hartree \\
\hline & 2 & -1801.965416 \\
\hline & $\mathbf{2}^{-}$ & -1802.077377 \\
\hline \multirow[t]{3}{*}{$\mathrm{C}$} & $2 \mu \mathrm{H}^{+}$ & -1802.372361 \\
\hline & $2 \mathbf{S H}^{\mathrm{in} *+}$ & -1802.324667 \\
\hline & $2 \mathrm{SH}^{*+}$ & -1802.322543 \\
\hline \multirow[t]{2}{*}{$\mathrm{CE}$} & $2 \mu \mathrm{H}$ & -1802.536058 \\
\hline & $2 \mu \mathbf{H}^{*}$ & -1802.51967 \\
\hline \multirow[t]{7}{*}{ CEE } & $2 \mathbf{t H}^{-}$ & -1802.631969 \\
\hline & $2 \mu \mathbf{H}^{*^{-}}$ & -1802.657054 \\
\hline & $\mathbf{2} \boldsymbol{\mu} \mathbf{H}^{-}(\mathrm{M}=3)$ & -1802.644348 \\
\hline & $2 \mu \mathrm{H}(\mathrm{CO})_{5}^{-}$ & -1689.313396 \\
\hline & $2 \mu \mathrm{H}(\mathrm{CO})_{5} *^{*^{-}}(\mathrm{M}=3)$ & -1689.329161 \\
\hline & $2 \mathrm{H}^{\mathrm{CO}^{-}}$ & -1802.651896 \\
\hline & $2 \mathrm{H}^{\mathrm{CO}^{-}}(\mathrm{M}=3)$ & -1802.627625 \\
\hline \multirow[t]{7}{*}{ CEEC } & $2 \mu \mathrm{HSH}^{*}$ & -1803.107672 \\
\hline & $2 \mu \mathrm{HtH}{ }^{\mathrm{S} *}$ & -1803.098947 \\
\hline & $2 \mu \mathrm{HtH}^{\mathrm{a} *}$ & -1803.098165 \\
\hline & $2 \mu \mathrm{HH}^{\mathrm{CO}}$ & -1803.101545 \\
\hline & $2 \mu \mathrm{HSH}{ }^{\mathrm{in} *}$ & -1803.105766 \\
\hline & $2 \mathbf{t H}_{2} *$ & -1803.068762 \\
\hline & 2tHSH ${ }^{\text {in } *}$ & -1803.095027 \\
\hline \multirow[t]{12}{*}{ CEECC } & $2 \mu \mathrm{HtH}^{\mathrm{S}} \mathrm{SH}^{*+}$ & -1803.512827 \\
\hline & $2 \mu \mathrm{HSH}_{2}{ }^{*+}$ & -1803.462960 \\
\hline & $2(\mathbf{t H})_{2} \mathrm{SH}^{+}$ & -1803.475865 \\
\hline & $2 \mu \mathrm{HtHSH}^{\#+}$ & -1803.473875 \\
\hline & $2 \mu \mathrm{HtH}^{\mathrm{a}} \mathrm{SH}^{*+}$ & -1803.510310 \\
\hline & $2 \mathrm{tH}_{2} \mathrm{tH}^{+}$ & -1803.478124 \\
\hline & $2 \mu \mathrm{H}_{2} \mathrm{SH}^{\#+}$ & -1803.498169 \\
\hline & $2 \mathbf{t H}_{2} \mathrm{SH}^{*+}$ & -1803.493391 \\
\hline & $2 \mathbf{t H}_{2} \mathrm{SH}^{\mathrm{in} *+}$ & -1803.493518 \\
\hline & $2 \mathbf{t} \mathbf{H}_{2} \boldsymbol{\mu} \mathbf{H}^{*+}$ & -1803.485311 \\
\hline & $2 \mu \mathrm{HtH}^{\mathrm{S}} \mathrm{SH}{ }^{\mathrm{in} *+}$ & -1803.510134 \\
\hline & $2 \mu \mathrm{HtH}_{2}{ }^{*+}$ & -1803.486848 \\
\hline State & Species & Solvent corrected free energy, hartree \\
\hline
\end{tabular}




\begin{tabular}{|c|c|c|}
\hline & 3 & -1954.367282 \\
\hline $\mathrm{C}$ & $3 \mu \mathbf{H}^{+}$ & -1954.772079 \\
\hline \multirow[t]{2}{*}{$\mathrm{CE}$} & $3 \mu \mathrm{H}$ & -1954.91548 \\
\hline & $3 \boldsymbol{\mu} \mathbf{H}^{*}$ & -1954.930588 \\
\hline \multirow[t]{3}{*}{ CEE } & $3 \boldsymbol{\mu} \mathbf{H}^{*-}$ & -1955.070113 \\
\hline & $\mathbf{3} \boldsymbol{\mu} \mathbf{H}^{-}$ & Converge to $3 \mu \mathbf{H}^{*^{-}}$ \\
\hline & $\mathbf{3} \boldsymbol{\mu} \mathbf{H}^{-}(\mathrm{M}=3)$ & Not converge \\
\hline \multicolumn{2}{|c|}{ Transitions } & Solvent corrected free energy, hartree \\
\hline \multicolumn{2}{|c|}{$\mathbf{T S}_{1}$} & -1803.08865 \\
\hline \multicolumn{2}{|c|}{$\mathbf{T S}_{\mathbf{2}}$} & -1803.093660 \\
\hline \multicolumn{2}{|c|}{$\mathbf{T S}_{3}$} & -1803.082571 \\
\hline \multicolumn{2}{|c|}{$\mathbf{T S}_{\mathbf{2}}$} & -1803.067248 \\
\hline \multicolumn{2}{|c|}{$\mathbf{T S}_{3}}$, & -1803.066847 \\
\hline \multicolumn{2}{|c|}{$\mathbf{T S}_{4}$} & -1803.485392 \\
\hline \multicolumn{2}{|c|}{$\mathbf{T S}_{5}$} & -1803.485746 \\
\hline \multicolumn{2}{|c|}{$\mathbf{T S}_{6}$} & -1803.474218 \\
\hline \multicolumn{2}{|c|}{$\mathbf{T S}_{7}$} & -1803.479029 \\
\hline \multicolumn{2}{|c|}{$\mathbf{T S}_{\mathbf{8}}$} & -1803.485871 \\
\hline \multicolumn{2}{|c|}{$\mathbf{T S}_{9}$} & -1803.471908 \\
\hline \multicolumn{2}{|c|}{$\mathbf{T S}_{10}$} & -1803.488604 \\
\hline \multicolumn{2}{|c|}{$\mathbf{T S}_{11}$} & -1802.288697 \\
\hline \multicolumn{2}{|c|}{$\mathbf{T S}_{12}$} & -1803.456937 \\
\hline \multicolumn{2}{|c|}{$\mathbf{T S}_{13}$} & -1803.480899 \\
\hline \multicolumn{2}{|c|}{$\mathbf{T S}_{14}$} & -1803.488076 \\
\hline \multicolumn{2}{|c|}{$\mathbf{T S}_{15}$} & -1802.305989 \\
\hline \multicolumn{2}{|c|}{ Species } & Solvent corrected free energy, hartree \\
\hline \multicolumn{2}{|c|}{$\mathrm{CO}$} & -113.324167 \\
\hline \multicolumn{2}{|c|}{$\mathrm{H}_{2}$} & -1.179982 \\
\hline \multicolumn{2}{|c|}{ HOTf } & -962.007113 \\
\hline \multicolumn{2}{|c|}{$\mathrm{OTf}^{-}$} & -961.581848 \\
\hline \multicolumn{2}{|c|}{$\left[\left(\mathrm{Et}_{3} \mathrm{Si}\right)_{2} \mathrm{H}\right]^{+}$} & -1054.506994 \\
\hline \multicolumn{2}{|c|}{$\left(\mathrm{Et}_{3} \mathrm{Si}\right)_{2}$} & -1054.090119 \\
\hline
\end{tabular}


Table S8. Summary of solvent corrected free energy for complex 2 and its related protonated/reduced species computed using BP86 functional.

\begin{tabular}{|c|c|c|c|}
\hline State & Species & Solvent corrected free energy, hartree & $\Delta G^{\text {state }}, \mathrm{kcal} / \mathrm{mol}$ \\
\hline \multirow[t]{2}{*}{$\mathrm{CE}$} & $2 \mu \mathrm{H}$ & -1802.954323 & 0 \\
\hline & $2 \mu \mathbf{H}^{*}$ & -1802.934377 & 12.52 \\
\hline \multirow[t]{7}{*}{ CEE } & $2 \mu H^{-\S}$ & -1803.073148 & 12.41 \\
\hline & $2 \mu \mathbf{H}^{*^{-}}$ & -1803.092938 & 0 \\
\hline & $2 \boldsymbol{\mu} \dot{H}^{-}(\mathrm{M}=3)$ & -1803.065828 & 17.01 \\
\hline & $2 \mu \mathrm{H}(\mathrm{CO})_{5}^{-}$ & -1689.740240 & 14.97 \\
\hline & $2 \mu \mathrm{H}(\mathrm{CO})_{5} *^{-}(\mathrm{M}=3)$ & -1689.731112 & 20.70 \\
\hline & $2 \mathrm{H}^{\mathrm{CO}-}$ & -1803.088513 & 2.78 \\
\hline & $2 \mathbf{H}^{\mathrm{CO}^{-}}(\mathrm{M}=3)$ & -1803.047544 & 28.48 \\
\hline \multirow[t]{5}{*}{ CEEC } & $2 \mu \mathrm{HSH}^{*}$ & -1803.536414 & 0 \\
\hline & $2 \mu \mathrm{HtH}^{\mathrm{S}} *$ & -1803.519720 & 10.48 \\
\hline & $2 \mu \mathrm{HtH}^{\mathrm{a} *}$ & -1803.517043 & 12.16 \\
\hline & $2 \mu \mathrm{HH}^{\mathrm{CO}}$ & -1803.522749 & 8.57 \\
\hline & 2 tHSH $^{\text {in } *}$ & -1803.523980 & 7.8 \\
\hline \multirow[t]{8}{*}{ CEECC } & $2 \mu \mathrm{HtH}^{\mathrm{SSH}}{ }^{*+}$ & -1803.923150 & 0 \\
\hline & $2 \mu \mathrm{HSH}_{2}{ }^{*+}$ & -1803.889558 & 21.08 \\
\hline & $2(\mathbf{t H})_{2} \mathrm{SH}^{+}$ & -1803.885910 & 23.37 \\
\hline & $2 \mu$ HtHSH $^{\#+}$ & -1803.873881 & 30.92 \\
\hline & $2 \mu \mathrm{HtH}^{\mathrm{a}} \mathrm{SH}^{*+}$ & -1803.919297 & 2.42 \\
\hline & $2 \mathrm{tH}_{2} \mathbf{t H}^{+}$ & -1803.887179 & 22.57 \\
\hline & $2 \mu \mathrm{H}_{2} \mathrm{SH}^{\#+}$ & -1803.896246 & 16.88 \\
\hline & $\mathrm{CO}$ & -113.328835 & \\
\hline
\end{tabular}

\footnotetext{
${ }^{\S}$ When $\mathbf{2} \boldsymbol{\mu} \mathbf{H}^{-}$was optimized using B3LYP functional, the converged structure was asymmetric.

The $\mu-\mathrm{H}$ was shifted toward one of the Fe center, leaving a very long Fe $\bullet \bullet H$ distance. We assign the structure to $\mathbf{2} \mathbf{t H}^{-}$. When BP86 functional was used for the optimization of $\mathbf{2} \boldsymbol{\mu} \mathbf{H}^{-}$, the hydride remained the bridging manner. We keep the assignment of the structure to $2 \mu \mathbf{H}^{-}$.
} 
Table S9. Cartesian coordinates for DFT geometry-optimized species using B3LYP.

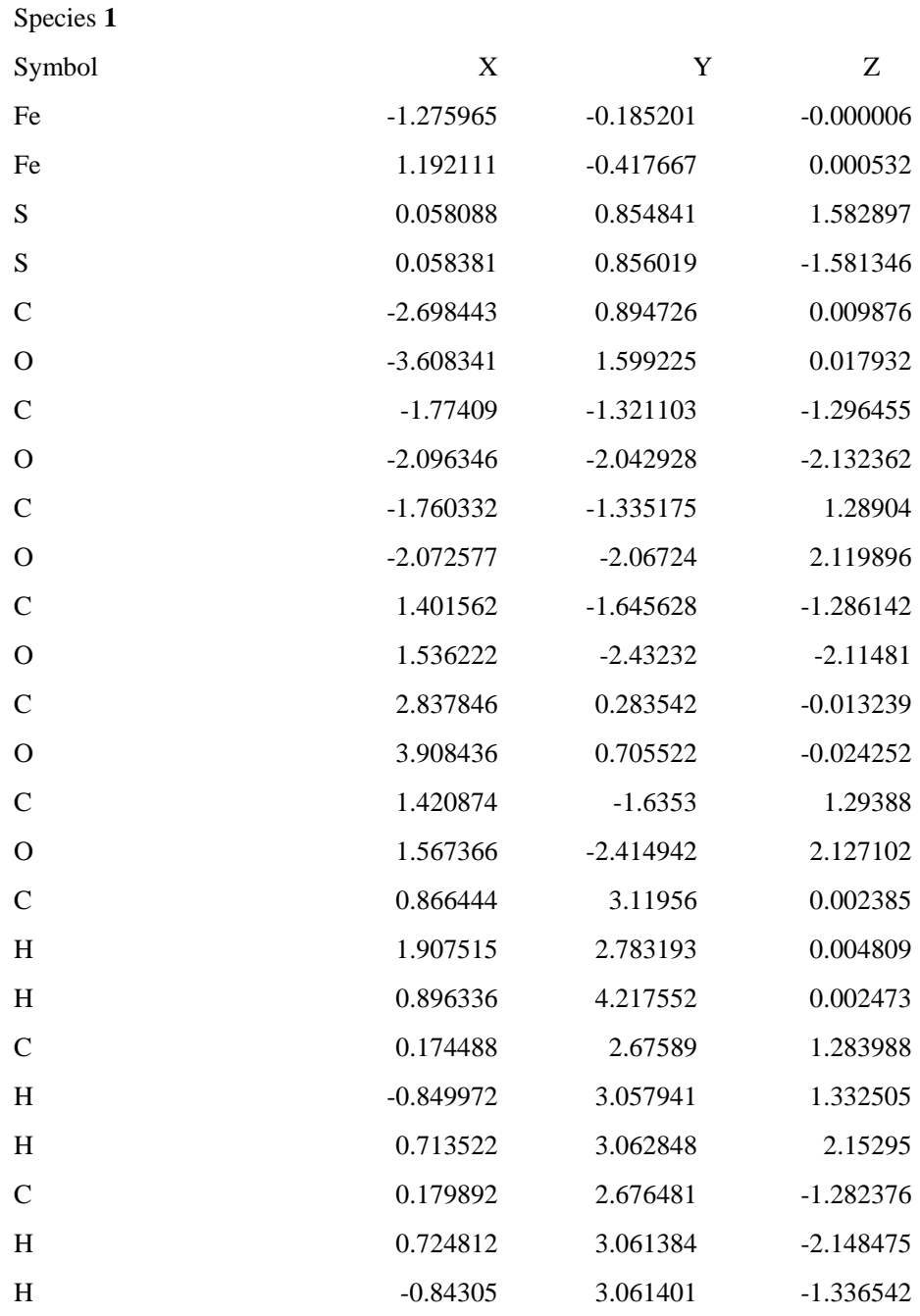

$\begin{array}{lrrr}\text { Species } \mathbf{1} \boldsymbol{\mu} \mathbf{H}^{+} & & & \\ \text {Symbol } & \mathrm{X} & \mathrm{Y} & \mathrm{Z} \\ \mathrm{Fe} & -1.312264 & -0.349739 & 0.000026 \\ \mathrm{Fe} & 1.265964 & -0.409452 & -0.000036 \\ \mathrm{~S} & -0.002043 & 0.744435 & 1.580579 \\ \mathrm{~S} & -0.002157 & 0.744286 & -1.580704 \\ \mathrm{C} & -2.67955 & 0.839568 & -0.000104 \\ \mathrm{O} & -3.525074 & 1.606334 & -0.000213 \\ \mathrm{C} & -1.994331 & -1.393787 & -1.340859 \\ \mathrm{O} & -2.396897 & -2.048632 & -2.18201 \\ \mathrm{C} & -1.994235 & -1.393373 & 1.341258 \\ \mathrm{O} & -2.396776 & -2.047949 & 2.182631 \\ \mathrm{C} & 1.866942 & -1.501399 & -1.343616 \\ \mathrm{O} & 2.216632 & -2.18442 & -2.185349\end{array}$




$\begin{array}{lrrr}\mathrm{C} & 2.758139 & 0.6232 & -0.000017 \\ \mathrm{O} & 3.719886 & 1.237201 & 0.000041 \\ \mathrm{C} & 1.867009 & -1.501343 & 1.343543 \\ \mathrm{O} & 2.216724 & -2.184324 & 2.185293 \\ \mathrm{C} & 0.565279 & 3.098601 & -0.000174 \\ \mathrm{H} & 1.645061 & 2.934647 & -0.000328 \\ \mathrm{H} & 0.427999 & 4.187446 & -0.00021 \\ \mathrm{C} & -0.044656 & 2.572315 & 1.292216 \\ \mathrm{H} & -1.095408 & 2.859548 & 1.385944 \\ \mathrm{H} & 0.48717 & 2.987806 & 2.15124 \\ \mathrm{C} & -0.045061 & 2.57217 & -1.292317 \\ \mathrm{H} & 0.486313 & 2.987786 & -2.151557 \\ \mathrm{H} & -1.095926 & 2.859133 & -1.38561 \\ \mathrm{H} & -0.057384 & -1.41831 & 0.000063\end{array}$

Species $\mathbf{1} \boldsymbol{\mu H}$

Symbol

$\mathrm{Fe}$

$\mathrm{Fe}$

S

S

C

O

C

$\mathrm{O}$

C

O

C

O

C

O

C

O

C

$\mathrm{H}$

$\mathrm{H}$

C

$\mathrm{H}$

$\mathrm{H}$

C

$\mathrm{H}$

$\mathrm{H}$

H

mbol

(

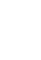

(

(

(

(

(

(

O

C

C

H

H

C

$\mathrm{H}$

H

C

H

$\begin{array}{rrr}X & Y & Z \\ 1.392318 & -0.388002 & -0.000031 \\ -1.375344 & -0.371241 & 0.000127 \\ 0.02719 & 0.697761 & -1.614833 \\ 0.027314 & 0.698164 & 1.614595 \\ 2.72857 & 0.868633 & -0.00027 \\ 3.574638 & 1.644343 & -0.000437 \\ 2.027674 & -1.421418 & 1.319119 \\ 2.404782 & -2.090331 & 2.172698 \\ 2.027647 & -1.421776 & -1.318909 \\ 2.404747 & -2.090919 & -2.172312 \\ -1.956494 & -1.463122 & 1.308383 \\ -2.327856 & -2.147004 & 2.155221 \\ -2.85611 & 0.767925 & 0.00024 \\ -3.817477 & 1.397912 & 0.000421 \\ -1.956751 & -1.463209 & -1.307935 \\ -2.328269 & -2.14712 & -2.15468 \\ -0.444044 & 3.050158 & -0.000445 \\ -1.524721 & 2.889369 & -0.000443 \\ -0.298698 & 4.139388 & -0.000573 \\ 0.157557 & 2.517181 & -1.29846 \\ 1.220227 & 2.769646 & -1.367424 \\ -0.344807 & 2.985775 & -2.149183 \\ 0.157547 & 2.517513 & 1.297714 \\ -0.344856 & 2.986297 & 2.148309 \\ 1.220199 & 2.770058 & 1.366642 \\ 0.16116 & -1.381367 & 0.000081\end{array}$




\begin{tabular}{|c|c|c|c|}
\hline Species & & & \\
\hline Symbol & $\mathrm{X}$ & Y & \\
\hline $\mathrm{Fe}$ & 1.053178 & -0.752916 & -0.120981 \\
\hline $\mathrm{Fe}$ & -1.616189 & -0.100892 & 0.024795 \\
\hline S & -0.083269 & 0.735267 & -1.483096 \\
\hline S & 1.780035 & 0.845201 & 1.508463 \\
\hline $\mathrm{C}$ & 2.668927 & -0.403888 & -0.844347 \\
\hline $\mathrm{O}$ & 3.690372 & -0.146109 & -1.29645 \\
\hline $\mathrm{C}$ & 1.605516 & -1.934154 & 1.128809 \\
\hline $\mathrm{O}$ & 1.932655 & -2.667808 & 1.944332 \\
\hline $\mathrm{C}$ & 0.544359 & -2.045251 & -1.294598 \\
\hline $\mathrm{O}$ & 0.244173 & -2.86778 & -2.032085 \\
\hline $\mathrm{C}$ & -1.759624 & 1.068688 & 1.470316 \\
\hline $\mathrm{O}$ & -1.88712 & 1.756267 & 2.380329 \\
\hline $\mathrm{C}$ & -3.011665 & 0.505057 & -0.935512 \\
\hline $\mathrm{O}$ & -3.892291 & 0.912564 & -1.553084 \\
\hline $\mathrm{C}$ & -2.512845 & -1.482196 & 0.753683 \\
\hline $\mathrm{O}$ & -3.054596 & -2.377759 & 1.231632 \\
\hline $\mathrm{H}$ & -0.335802 & -0.885618 & 0.73928 \\
\hline $\mathrm{C}$ & 0.403428 & 2.513039 & -1.334509 \\
\hline $\mathrm{H}$ & 1.226218 & 2.628076 & -2.047296 \\
\hline $\mathrm{C}$ & 2.035813 & 2.458521 & 0.662077 \\
\hline $\mathrm{H}$ & 2.846115 & 2.379378 & -0.071353 \\
\hline $\mathrm{C}$ & 0.793187 & 3.081577 & 0.024371 \\
\hline $\mathrm{H}$ & 2.394855 & 3.112225 & 1.46314 \\
\hline $\mathrm{H}$ & -0.457323 & 3.051744 & -1.740607 \\
\hline $\mathrm{H}$ & 0.996601 & 4.14838 & -0.14725 \\
\hline $\mathrm{H}$ & -0.048753 & 3.033976 & 0.719912 \\
\hline
\end{tabular}

Species $\mathbf{1} \boldsymbol{\mu} \mathbf{H}^{-}$

$\begin{array}{lrrr}\text { Symbol } & \mathrm{X} & \mathrm{Y} & \mathrm{Z} \\ \mathrm{Fe} & 1.670072 & -0.328006 & 0.012272 \\ \mathrm{Fe} & -1.731567 & -0.354281 & -0.044892 \\ \mathrm{~S} & 0.007779 & 0.476963 & -1.485403 \\ \mathrm{~S} & 0.007324 & 0.353481 & 1.575595 \\ \mathrm{C} & 2.684142 & 1.185555 & 0.056711 \\ \mathrm{O} & 3.391607 & 2.093931 & 0.081917 \\ \mathrm{C} & 2.485053 & -1.208427 & 1.321595 \\ \mathrm{O} & 2.948265 & -1.819581 & 2.181262 \\ \mathrm{C} & 2.483917 & -1.131582 & -1.347575 \\ \mathrm{O} & 2.945053 & -1.69293 & -2.241542 \\ \mathrm{C} & -2.810133 & -0.783567 & 1.271311 \\ \mathrm{O} & -3.498396 & -1.059354 & 2.171199 \\ \mathrm{C} & -2.956279 & 0.633805 & -0.820496\end{array}$


$\mathrm{O}$
$\mathrm{C}$
$\mathrm{C}$
$\mathrm{H}$
$\mathrm{H}$
$\mathrm{C}$
$\mathrm{H}$
$\mathrm{H}$
$\mathrm{C}$
$\mathrm{H}$
$\mathrm{H}$
$\mathrm{H}$

C

$\mathrm{O}$

C

$\mathrm{H}$

$\mathrm{H}$

C

$\mathrm{H}$

$\mathrm{H}$

C

$\mathrm{H}$

$\mathrm{H}$

H

Species $\mathbf{1} \boldsymbol{\mu} \mathbf{H}^{-}(\mathbf{M}=3)$

Symbol

$\mathrm{Fe}$

$\mathrm{Fe}$

$\mathrm{S}$

S

C

$\mathrm{O}$

C

$\mathrm{O}$

C

$\mathrm{O}$

C

$\mathrm{O}$

C

$\mathrm{O}$

C

O

C

$\mathrm{H}$

$\mathrm{H}$

C

$\mathrm{H}$

H

C

$\mathrm{H}$

H

$\mathrm{H}$

Fe

$\begin{array}{rrr}-3.806565 & 1.178234 & -1.419634 \\ -1.567112 & -1.998582 & -0.655244 \\ -1.610692 & -3.066075 & -1.135981 \\ -0.567442 & 2.787007 & 0.156665 \\ -1.641166 & 2.584658 & 0.164852 \\ -0.446825 & 3.879968 & 0.195148 \\ -0.001165 & 2.31068 & -1.18657 \\ 1.022487 & 2.66481 & -1.339486 \\ -0.611345 & 2.736482 & -1.989699 \\ 0.032838 & 2.204211 & 1.43944 \\ -0.546805 & 2.573873 & 2.291785 \\ 1.065135 & 2.535137 & 1.586939 \\ 0.858659 & -1.590966 & -0.01727\end{array}$

\begin{tabular}{|c|c|c|}
\hline $\mathrm{X}$ & $\mathrm{Y}$ & $\mathrm{Z}$ \\
\hline 1.279427 & -0.623036 & 0.000184 \\
\hline-1.5931 & -0.171342 & -0.000072 \\
\hline 0.349464 & 0.806171 & -1.67934 \\
\hline 0.344657 & 0.80364 & 1.678859 \\
\hline 2.86948 & 0.263041 & 0.002966 \\
\hline 3.872759 & 0.828716 & 0.00485 \\
\hline 1.604881 & -1.769146 & 1.323434 \\
\hline 1.780944 & -2.510972 & 2.187431 \\
\hline 1.608253 & -1.766977 & -1.324116 \\
\hline 1.786578 & -2.507277 & -2.18896 \\
\hline-2.283559 & -1.151206 & 1.391888 \\
\hline-2.778981 & -1.785604 & 2.231681 \\
\hline-2.785319 & 1.216028 & 0.000185 \\
\hline-3.567325 & 2.077542 & 0.000198 \\
\hline-2.280773 & -1.147727 & -1.395832 \\
\hline-2.774804 & -1.779761 & -2.238211 \\
\hline 0.376426 & 3.136848 & 0.001187 \\
\hline-0.717732 & 3.100043 & -0.000772 \\
\hline 0.659898 & 4.200763 & 0.002451 \\
\hline 0.911007 & 2.523047 & -1.294043 \\
\hline 2.006713 & 2.551424 & -1.302216 \\
\hline 0.572073 & 3.126014 & -2.142985 \\
\hline 0.906498 & 2.521407 & 1.297473 \\
\hline 0.56429 & 3.123047 & 2.14604 \\
\hline 2.002162 & 2.550095 & 1.309661 \\
\hline-0.152565 & -1.287581 & -0.002137 \\
\hline
\end{tabular}

Species $\mathbf{1} \boldsymbol{\mu} \mathbf{H}^{*-}$ 


\begin{tabular}{|c|c|c|c|}
\hline Symbol & $\mathrm{X}$ & $\mathrm{Y}$ & $\mathrm{Z}$ \\
\hline $\mathrm{Fe}$ & 0.907537 & -0.777713 & -0.07553 \\
\hline $\mathrm{Fe}$ & -1.487296 & 0.151744 & 0.131634 \\
\hline$S$ & 0.087451 & 0.96645 & -1.418861 \\
\hline$S$ & 2.644573 & 0.446071 & 1.146886 \\
\hline $\mathrm{C}$ & 2.086274 & -1.349237 & -1.263851 \\
\hline $\mathrm{O}$ & 2.870579 & -1.69473 & -2.039065 \\
\hline $\mathrm{C}$ & 1.203412 & -2.050115 & 1.116559 \\
\hline $\mathrm{O}$ & 1.391517 & -2.857684 & 1.920598 \\
\hline $\mathrm{C}$ & -0.642229 & -1.582721 & -0.727678 \\
\hline $\mathrm{O}$ & -1.119801 & -2.518504 & -1.280399 \\
\hline $\mathrm{C}$ & -1.761329 & 1.710291 & 0.993456 \\
\hline $\mathrm{O}$ & -2.029419 & 2.69546 & 1.534571 \\
\hline $\mathrm{C}$ & -2.774961 & 0.161265 & -1.094591 \\
\hline $\mathrm{O}$ & -3.610076 & 0.178891 & -1.89368 \\
\hline $\mathrm{C}$ & -2.326721 & -0.888776 & 1.287755 \\
\hline $\mathrm{O}$ & -2.843199 & -1.574839 & 2.061591 \\
\hline $\mathrm{H}$ & -0.15173 & -0.05599 & 1.054467 \\
\hline $\mathrm{C}$ & 0.6779 & 2.66685 & -0.995957 \\
\hline $\mathrm{H}$ & 1.309567 & 2.952052 & -1.844519 \\
\hline $\mathrm{C}$ & 2.763165 & 2.134086 & 0.421527 \\
\hline $\mathrm{H}$ & 3.246704 & 2.085579 & -0.562096 \\
\hline $\mathrm{C}$ & 1.439887 & 2.901197 & 0.305727 \\
\hline $\mathrm{H}$ & 3.441344 & 2.683717 & 1.082426 \\
\hline $\mathrm{H}$ & -0.214471 & 3.299139 & -1.031836 \\
\hline $\mathrm{H}$ & 1.652668 & 3.98047 & 0.335841 \\
\hline $\mathrm{H}$ & 0.808087 & 2.684117 & 1.172017 \\
\hline \multicolumn{4}{|c|}{ Species 2} \\
\hline Symbol & $\mathrm{X}$ & $\mathrm{Y}$ & $\mathrm{Z}$ \\
\hline $\mathrm{Fe}$ & -1.237849 & -0.177342 & 0.000051 \\
\hline $\mathrm{Fe}$ & 1.237851 & -0.177341 & -0.000062 \\
\hline$S$ & 0.000062 & 1.06239 & 1.498883 \\
\hline S & -0.00006 & 1.062243 & -1.499014 \\
\hline $\mathrm{C}$ & -2.78154 & 0.723597 & 0.000048 \\
\hline $\mathrm{O}$ & -3.77657 & 1.30079 & 0.00024 \\
\hline $\mathrm{C}$ & -1.587698 & -1.361344 & -1.296245 \\
\hline $\mathrm{O}$ & -1.812468 & -2.116441 & -2.134897 \\
\hline $\mathrm{C}$ & -1.587587 & -1.361216 & 1.296499 \\
\hline $\mathrm{O}$ & -1.812284 & -2.116198 & 2.135273 \\
\hline $\mathrm{C}$ & 1.587583 & -1.361341 & -1.296389 \\
\hline $\mathrm{O}$ & 1.812273 & -2.116435 & -2.135065 \\
\hline $\mathrm{C}$ & 2.781543 & 0.723596 & -0.000203 \\
\hline $\mathrm{O}$ & 3.776573 & 1.300788 & -0.000095 \\
\hline
\end{tabular}


C

O

C

H

H

C

H

H

Species $\mathbf{2} \boldsymbol{\mu} \mathbf{H}^{+}$

Symbol

$\mathrm{Fe}$

$\mathrm{Fe}$

S

S

C

$\mathrm{O}$

C

$\mathrm{O}$

C

$\mathrm{O}$

C

O

C

$\mathrm{O}$

C

O

C

$\mathrm{H}$

H

C

H

H

H

Species $\mathbf{2 S H}^{\mathrm{in} *+}$

$\begin{array}{llll}\text { Symbol } & \mathrm{X} & \mathrm{Y} & \mathrm{Z} \\ \mathrm{Fe} & -1.544644 & 0.057294 & -0.241158 \\ \mathrm{Fe} & 0.957699 & -0.537097 & -0.127434 \\ \mathrm{~S} & 2.22785 & 0.983633 & 1.234207 \\ \mathrm{~S} & 0.099206 & 1.2832 & -1.293737 \\ \mathrm{C} & -0.897145 & 0.123285 & 1.385584 \\ \mathrm{O} & -0.780512 & 0.302446 & 2.528167 \\ \mathrm{C} & -2.827809 & 1.329022 & -0.324136\end{array}$

1.296355

2.135106

0.765656

1.162846

1.162974

$-0.765955$

$-1.163193$

$-1.163321$

3.261463

Z

$\begin{array}{lll}-1.285756 & -0.262475 & 0.000055\end{array}$

$\begin{array}{lll}1.285863 & -0.262194 & -0.000048\end{array}$

$\begin{array}{lll}0.000049 & 0.943593 & 1.50474\end{array}$

$\begin{array}{lll}-0.000075 & 0.94342 & -1.504873\end{array}$

$\begin{array}{lll}-2.701963 & 0.871046 & 0.000112\end{array}$

$\begin{array}{lll}-3.580903 & 1.597685 & 0.00016\end{array}$

$\begin{array}{lll}-1.926584 & -1.324402 & -1.344351\end{array}$

$\begin{array}{lll}-2.303065 & -1.989758 & -2.189048\end{array}$

$\begin{array}{lll}-1.926433 & -1.324282 & 1.344627\end{array}$

$\begin{array}{lll}-2.30282 & -1.989579 & 2.189413\end{array}$

$1.926811-1.324003 \quad-1.344511$

$\begin{array}{lll}2.303388 & -1.989252 & -2.189247\end{array}$

$\begin{array}{lll}2.701909 & 0.871516 & -0.000081\end{array}$

$\begin{array}{lll}3.580816 & 1.598192 & -0.000072\end{array}$

$\begin{array}{lll}1.926866 & -1.323906 & 1.34446\end{array}$

$\begin{array}{lll}2.303472 & -1.989104 & 2.189224\end{array}$

$\begin{array}{lll}-0.000717 & 2.634591 & 0.766324\end{array}$

$\begin{array}{lll}-0.882732 & 3.1391 & 1.166467\end{array}$

$\begin{array}{lll}0.880734 & 3.140158 & 1.166411\end{array}$

$\begin{array}{lll}-0.000823 & 2.634479 & -0.766706\end{array}$

$\begin{array}{lll}0.880768 & 3.139913 & -1.166625\end{array}$

$\begin{array}{lll}-0.882746 & 3.139042 & -1.166979\end{array}$

$\begin{array}{lll}0.000135 & -1.304714 & 0.000016\end{array}$

$\begin{array}{rrr}1.587704 & -1.361216 & 1.296355 \\ 1.812474 & -2.116202 & 2.135106 \\ -0.000059 & 2.756626 & 0.765656 \\ -0.883938 & 3.261379 & 1.162846 \\ 0.883648 & 3.261581 & 1.162974 \\ 0.000052 & 2.756551 & -0.765955 \\ 0.883928 & 3.261271 & -1.163193 \\ -0.883658 & 3.261463 & -1.163321\end{array}$

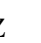

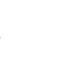




$\begin{array}{llll}\mathrm{O} & -3.602988 & 2.169717 & -0.27972 \\ \mathrm{C} & -2.699533 & -1.266958 & 0.246449 \\ \mathrm{O} & -3.428739 & -2.102106 & 0.523019 \\ \mathrm{C} & -0.043658 & -1.634861 & -1.150991 \\ \mathrm{O} & -0.582021 & -2.360278 & -1.86412 \\ \mathrm{C} & 2.452401 & -0.752071 & -1.149573 \\ \mathrm{O} & 3.362276 & -0.912264 & -1.819394 \\ \mathrm{C} & 1.285744 & -1.867221 & 1.079668 \\ \mathrm{O} & 1.503845 & -2.689596 & 1.840822 \\ \mathrm{C} & 2.01369 & 2.556767 & 0.291705 \\ \mathrm{H} & 2.284281 & 3.374132 & 0.964789 \\ \mathrm{H} & 2.755531 & 2.503194 & -0.507644 \\ \mathrm{C} & 0.608905 & 2.732944 & -0.252752 \\ \mathrm{H} & 0.580321 & 3.603957 & -0.912886 \\ \mathrm{H} & -0.11824 & 2.879716 & 0.545709 \\ \mathrm{H} & 1.395308 & 1.255772 & 2.259949\end{array}$

Species 2SH*+

$\begin{array}{lrrr}\text { Symbol } & \mathrm{X} & \mathrm{Y} & \mathrm{Z} \\ \mathrm{Fe} & 1.544453 & -0.073512 & -0.235068 \\ \mathrm{Fe} & -0.956669 & 0.545205 & -0.139109 \\ \mathrm{~S} & -2.066857 & -0.994246 & 1.342022 \\ \mathrm{~S} & -0.108138 & -1.291014 & -1.285258 \\ \mathrm{C} & 0.924186 & -0.108856 & 1.406586 \\ \mathrm{O} & 0.802622 & -0.248163 & 2.551167 \\ \mathrm{C} & 2.824959 & -1.349896 & -0.313433 \\ \mathrm{O} & 3.598452 & -2.191923 & -0.262109 \\ \mathrm{C} & 2.701236 & 1.25858 & 0.221782 \\ \mathrm{O} & 3.431093 & 2.100573 & 0.476017 \\ \mathrm{C} & 0.02981 & 1.626164 & -1.188193 \\ \mathrm{O} & 0.573599 & 2.335457 & -1.913132 \\ \mathrm{C} & -2.469326 & 0.7217 & -1.130613 \\ \mathrm{O} & -3.401421 & 0.844934 & -1.778679 \\ \mathrm{C} & -1.263122 & 1.894089 & 1.05066 \\ \mathrm{O} & -1.469869 & 2.731522 & 1.799031 \\ \mathrm{C} & -2.049173 & -2.526437 & 0.296505 \\ \mathrm{H} & -2.339865 & -3.358772 & 0.94205 \\ \mathrm{H} & -2.781858 & -2.408037 & -0.502746 \\ \mathrm{C} & -0.648649 & -2.735193 & -0.248386 \\ \mathrm{H} & -0.643782 & -3.603845 & -0.910755 \\ \mathrm{H} & 0.078224 & -2.902801 & 0.547032 \\ \mathrm{H} & -3.37052 & -0.686499 & 1.196995\end{array}$

Species $\mathbf{2} \boldsymbol{\mu H}$ 


$\begin{array}{lrrr}\text { Symbol } & \mathrm{X} & \mathrm{Y} & \mathrm{Z} \\ \mathrm{Fe} & -1.370104 & -0.270388 & 0.000563 \\ \mathrm{Fe} & 1.371004 & -0.263611 & -0.000697 \\ \mathrm{~S} & -0.001576 & 0.912895 & 1.529125 \\ \mathrm{~S} & -0.006216 & 0.912978 & -1.529385 \\ \mathrm{C} & -2.769031 & 0.942109 & 0.002774 \\ \mathrm{O} & -3.642371 & 1.686512 & 0.004159 \\ \mathrm{C} & -1.970001 & -1.33095 & -1.315517 \\ \mathrm{O} & -2.336705 & -2.004464 & -2.171305 \\ \mathrm{C} & -1.968491 & -1.330908 & 1.317616 \\ \mathrm{O} & -2.333898 & -2.004942 & 2.17352 \\ \mathrm{C} & 1.969856 & -1.329691 & -1.315473 \\ \mathrm{O} & 2.343476 & -2.001997 & -2.169977 \\ \mathrm{C} & 2.778511 & 0.948859 & -0.001902 \\ \mathrm{O} & 3.653449 & 1.692075 & -0.002125 \\ \mathrm{C} & 1.970272 & -1.330882 & 1.312724 \\ \mathrm{O} & 2.344434 & -2.00339 & 2.166859 \\ \mathrm{C} & 0.007983 & 2.595121 & 0.769511 \\ \mathrm{H} & -0.855101 & 3.127955 & 1.176773 \\ \mathrm{H} & 0.90902 & 3.093012 & 1.136422 \\ \mathrm{C} & -0.027652 & 2.595065 & -0.769789 \\ \mathrm{H} & 0.832829 & 3.131922 & -1.177287 \\ \mathrm{H} & -0.931024 & 3.088862 & -1.136492 \\ \mathrm{H} & -0.030219 & -1.234411 & -0.000449\end{array}$

$\begin{array}{lrrr}\text { Species } 2 \mu \mathbf{H}^{*} & \mathrm{Y} & \mathrm{Z} \\ \text { Symbol } & \mathrm{X} & -0.562268 & -0.074076 \\ \mathrm{Fe} & 1.097971 & -0.051288 & 0.005879 \\ \mathrm{Fe} & -1.5497 & 1.021756 & -1.362042 \\ \mathrm{~S} & -0.00221 & 1.198314 & 1.336101 \\ \mathrm{~S} & 1.864093 & -0.313599 & -0.894075 \\ \mathrm{C} & 2.691765 & -0.135304 & -1.403486 \\ \mathrm{O} & 3.702005 & -1.674509 & 1.215125 \\ \mathrm{C} & 1.685764 & -2.355345 & 2.065565 \\ \mathrm{O} & 2.037761 & -1.886123 & -1.168204 \\ \mathrm{C} & 0.517998 & -2.735274 & -1.863623 \\ \mathrm{O} & 0.189176 & 1.181198 & 1.366037 \\ \mathrm{C} & -1.869849 & 1.935571 & 2.210248 \\ \mathrm{O} & -2.053918 & 0.375846 & -1.067859 \\ \mathrm{C} & -2.936726 & -1.745275 \\ \mathrm{O} & -3.815565 & 0.675729 & 0.760733 \\ \mathrm{C} & -2.373808 & -1.46533 & 1.262874 \\ \mathrm{O} & -2.873804 & -2.371074 & 0.860063 \\ \mathrm{H} & -0.243332 & -0.623527 & \end{array}$


C

$\mathrm{H}$

$\mathrm{H}$

C

$\mathrm{H}$

H

Species 2tH

Symbol

$\mathrm{Fe}$

$\mathrm{Fe}$

S

S

C

O

C

O

C

$\mathrm{O}$

C

$\mathrm{O}$

C

O

C

$\mathrm{O}$

C

$\mathrm{H}$

H

C

H

H

H

Species $\mathbf{2} \boldsymbol{\mu} \mathbf{H}^{*}$

Symbol

$\mathrm{Fe}$

$\mathrm{Fe}$

$\mathrm{S}$

S

C

$\mathrm{O}$

C

$\mathrm{O}$

C

$\begin{array}{rrr}0.467979 & 2.652673 & -0.596932 \\ 0.50314 & 3.36887 & -1.423498 \\ -0.32687 & 2.945536 & 0.088592 \\ 1.80665 & 2.572745 & 0.115575 \\ 1.97615 & 3.498462 & 0.677155 \\ 2.621854 & 2.470178 & -0.60695\end{array}$

\begin{tabular}{rrr}
$X$ & $Y$ & $Z$ \\
-1.72373 & -0.211607 & -0.037994 \\
1.62751 & -0.262403 & 0.03391 \\
-0.003248 & 0.652642 & 1.508351 \\
0.008374 & 0.668819 & -1.444422 \\
-2.925217 & 0.980423 & -0.533176 \\
-3.806157 & 1.631833 & -0.95078 \\
-1.741606 & -1.739222 & -0.90159 \\
-1.858432 & -2.707882 & -1.55231 \\
-2.683656 & -0.851933 & 1.284181 \\
-3.289691 & -1.269676 & 2.188244 \\
2.381764 & -1.167094 & -1.292849 \\
2.805189 & -1.789641 & -2.164928 \\
2.748863 & 1.187175 & 0.040325 \\
3.504822 & 2.054541 & 0.051011 \\
2.365396 & -1.157827 & 1.37315 \\
2.779209 & -1.773404 & 2.255045 \\
-0.018172 & 2.364628 & 0.811423 \\
-0.922495 & 2.844309 & 1.194203 \\
0.846109 & 2.908251 & 1.204604 \\
-0.005744 & 2.373921 & -0.735689 \\
0.869419 & 2.908812 & -1.11553 \\
-0.898852 & 2.865772 & -1.128835 \\
0.716208 & -1.452833 & 0.043993 \\
\hline & & $Y$ \\
\hline
\end{tabular}

$\begin{array}{lll}0.716208 & -1.452833 & 0.043993\end{array}$

$\begin{array}{lrrr}\text { Symbol } & \mathrm{X} & \mathrm{Y} & \mathrm{Z} \\ \mathrm{Fe} & 1.131646 & -0.463846 & -0.023348 \\ \mathrm{Fe} & -1.40236 & -0.010159 & 0.080625 \\ \mathrm{~S} & 0.052008 & 1.034055 & -1.446997 \\ \mathrm{~S} & 2.367897 & 1.394195 & 0.921912 \\ \mathrm{C} & 2.430073 & -0.884871 & -1.144126 \\ \mathrm{O} & 3.282063 & -1.139915 & -1.88208 \\ \mathrm{C} & 1.701172 & -1.454368 & 1.325044 \\ \mathrm{O} & 2.07096 & -2.070491 & 2.229729 \\ \mathrm{C} & -0.1687 & -1.64484 & -0.602284\end{array}$




$\begin{array}{lrrr}\mathrm{O} & -0.451403 & -2.702943 & -1.054027 \\ \mathrm{C} & -2.070456 & 1.500861 & 0.789031 \\ \mathrm{O} & -2.608218 & 2.4316 & 1.215767 \\ \mathrm{C} & -2.577603 & -0.386711 & -1.199694 \\ \mathrm{O} & -3.349473 & -0.618145 & -2.029305 \\ \mathrm{C} & -2.066949 & -1.116163 & 1.288806 \\ \mathrm{O} & -2.467036 & -1.83673 & 2.099528 \\ \mathrm{H} & -0.091318 & 0.110611 & 1.045236 \\ \mathrm{C} & 0.598998 & 2.68661 & -0.820998 \\ \mathrm{H} & 1.401894 & 2.990941 & -1.499682 \\ \mathrm{H} & -0.239204 & 3.380728 & -0.951073 \\ \mathrm{C} & 1.094534 & 2.68179 & 0.619143 \\ \mathrm{H} & 0.255206 & 2.52823 & 1.301701 \\ \mathrm{H} & 1.531938 & 3.660777 & 0.849547\end{array}$

Species $\mathbf{2} \boldsymbol{\mu} \mathbf{H}^{-}(\mathrm{M}=3)$

Symbol

$\mathrm{Fe}$

$\mathrm{Fe}$

S

S

C

O

C

$\mathrm{O}$

C

O

C

O

C

O

C

O

C

$\mathrm{H}$

$\mathrm{H}$

C

$\mathrm{H}$

$\mathrm{H}$

$\mathrm{H}$

Species $2 \mu \mathbf{H}(\mathbf{C O})_{5}$

Symbol

$\mathrm{Fe}$

$\begin{array}{rrr}X & Y & Z \\ -1.320264 & -0.424362 & -0.010454 \\ 1.556607 & -0.136677 & 0.025073 \\ -0.648826 & 1.221849 & 1.571475 \\ -0.157833 & 0.975408 & -1.574538 \\ -2.923063 & 0.416052 & -0.310965 \\ -3.931576 & 0.933986 & -0.507264 \\ -1.466338 & -1.675169 & -1.27061 \\ -1.519224 & -2.482201 & -2.091318 \\ -1.806204 & -1.459735 & 1.350775 \\ -2.092098 & -2.117467 & 2.253345 \\ 2.258891 & -1.337289 & -1.167836 \\ 2.7075 & -2.11646 & -1.905258 \\ 2.821227 & 1.175854 & -0.084701 \\ 3.641518 & 1.997091 & -0.156346 \\ 2.095552 & -0.911987 & 1.592498 \\ 2.510317 & -1.4239 & 2.550683 \\ -0.961805 & 2.708385 & 0.534212 \\ -2.038061 & 2.812721 & 0.371947 \\ -0.633148 & 3.582471 & 1.108543 \\ -0.23116 & 2.664525 & -0.809911 \\ 0.803685 & 2.994784 & -0.690171 \\ -0.721811 & 3.336047 & -1.522257 \\ 0.096859 & -1.10732 & 0.24936\end{array}$

$\begin{array}{rrr}X & Y & Z \\ -1.08323 & -0.467244 & 0.024225\end{array}$




$\begin{array}{lrrr}\mathrm{Fe} & 1.47456 & 0.094308 & -0.006142 \\ \mathrm{~S} & -0.004274 & 1.090279 & 1.4687 \\ \mathrm{~S} & -0.007383 & 0.956191 & -1.558048 \\ \mathrm{C} & -2.684169 & 0.314824 & -0.013113 \\ \mathrm{O} & -3.771336 & 0.71307 & -0.03149 \\ \mathrm{C} & -1.30889 & -1.709589 & -1.224431 \\ \mathrm{O} & -1.469196 & -2.487532 & -2.065696 \\ \mathrm{C} & -1.312348 & -1.595504 & 1.376135 \\ \mathrm{O} & -1.477759 & -2.298722 & 2.27996 \\ \mathrm{C} & 2.564263 & -0.448361 & -1.241521 \\ \mathrm{O} & 3.293828 & -0.805417 & -2.082932 \\ \mathrm{C} & 2.585383 & -0.30101 & 1.264754 \\ \mathrm{O} & 3.329972 & -0.556002 & 2.13016 \\ \mathrm{C} & -0.514785 & 2.666712 & 0.653092 \\ \mathrm{H} & -1.508695 & 2.918126 & 1.034308 \\ \mathrm{H} & 0.182545 & 3.433207 & 1.001715 \\ \mathrm{C} & -0.528 & 2.595193 & -0.882496 \\ \mathrm{H} & 0.151354 & 3.33637 & -1.312294 \\ \mathrm{H} & -1.531821 & 2.796097 & -1.267336 \\ \mathrm{H} & 0.665765 & -1.187739 & 0.068477\end{array}$

Species $\mathbf{2} \boldsymbol{\mu H}(\mathrm{CO})_{5}$ *- $^{-}(\mathrm{M}=3)$

Symbol

$\mathrm{Fe}$

$\mathrm{Fe}$

S

S

C

O

C

$\mathrm{O}$

C

O

C

O

C

O

C

$\mathrm{H}$

$\mathrm{H}$

C

$\mathrm{H}$

H

$\mathrm{H}$
X

$-1.438204$

1.325094

2.172079

$-0.019557$

$-2.77102$

$-3.639282$

$-2.154435$

$-2.527735$

$-1.805026$

$-2.020786$

1.659707

1.942506

2.159475

2.68854

1.636729

1.728182

2.320488

0.208586

$-0.042451$

$-0.493633$

$-0.162122$

$\begin{array}{rr}Y & Z \\ -0.152258 & 0.156891 \\ -0.371708 & -0.085811 \\ 1.473481 & 1.007794 \\ 0.989704 & -1.360273 \\ 1.039058 & -0.030053 \\ 1.792223 & -0.15273 \\ -1.541058 & -0.805969 \\ -2.450437 & -1.410995 \\ -0.651479 & 1.835265 \\ -1.000685 & 2.916913 \\ -1.417813 & -1.490204 \\ -2.043112 & -2.427862 \\ -1.430445 & 1.051459 \\ -2.131278 & 1.809999 \\ 2.846184 & -0.113266 \\ 3.789521 & 0.437784 \\ 2.887162 & -0.967386 \\ 2.672887 & -0.595259 \\ 3.413345 & -1.363005 \\ 2.775985 & 0.234564 \\ -1.091546 & 0.455202\end{array}$




\begin{tabular}{|c|c|c|c|}
\hline \multicolumn{4}{|l|}{ Species 2} \\
\hline Symbol & $\mathrm{X}$ & $\mathrm{Y}$ & $\mathrm{Z}$ \\
\hline $\mathrm{Fe}$ & 1.252262 & -0.37246 & -0.046693 \\
\hline $\mathrm{Fe}$ & -1.203966 & 0.093027 & 0.025416 \\
\hline S & 0.204524 & 1.162143 & -1.458968 \\
\hline S & 0.348576 & 1.072306 & 1.565745 \\
\hline $\mathrm{C}$ & 2.949745 & 0.155691 & -0.014306 \\
\hline $\mathrm{O}$ & 4.071329 & 0.442537 & 0.015096 \\
\hline $\mathrm{C}$ & 1.214148 & -1.66104 & 1.161435 \\
\hline $\mathrm{O}$ & 1.20328 & -2.494874 & 1.966614 \\
\hline $\mathrm{C}$ & 1.280999 & -1.524337 & -1.391696 \\
\hline $\mathrm{O}$ & 1.306967 & -2.268624 & -2.278208 \\
\hline $\mathrm{C}$ & -1.803688 & -1.054715 & 1.226641 \\
\hline $\mathrm{O}$ & -2.21062 & -1.806072 & 2.008307 \\
\hline $\mathrm{C}$ & -2.601338 & 1.137905 & -0.02425 \\
\hline $\mathrm{O}$ & -3.529939 & 1.835407 & -0.06222 \\
\hline $\mathrm{C}$ & -1.900773 & -1.003309 & -1.388369 \\
\hline $\mathrm{O}$ & -2.678125 & -1.949245 & -1.347029 \\
\hline $\mathrm{C}$ & 0.490816 & 2.801338 & -0.653887 \\
\hline $\mathrm{H}$ & 1.278562 & 3.31572 & -1.212262 \\
\hline $\mathrm{H}$ & -0.43682 & 3.364378 & -0.78349 \\
\hline $\mathrm{C}$ & 0.86046 & 2.685258 & 0.827104 \\
\hline $\mathrm{H}$ & 0.384449 & 3.480729 & 1.408965 \\
\hline $\mathrm{H}$ & 1.941521 & 2.768076 & 0.96358 \\
\hline $\mathrm{H}$ & -1.518372 & -0.70861 & -2.408529 \\
\hline \multicolumn{4}{|c|}{ Species $2 \mathbf{H}^{\mathbf{C O}-}(\mathbf{M}=3)$} \\
\hline Symbol & $\mathrm{X}$ & $\mathrm{Y}$ & $\mathrm{Z}$ \\
\hline $\mathrm{Fe}$ & 1.462416 & -0.281467 & 0.044301 \\
\hline $\mathrm{Fe}$ & -1.549972 & 0.106558 & 0.013605 \\
\hline S & 0.15045 & 0.664899 & -1.664324 \\
\hline$S$ & 0.19992 & 1.209434 & 1.340965 \\
\hline $\mathrm{C}$ & 3.029779 & 0.694033 & -0.134777 \\
\hline $\mathrm{O}$ & 3.960739 & 1.364791 & -0.247359 \\
\hline $\mathrm{C}$ & 1.849794 & -1.203423 & 1.534363 \\
\hline $\mathrm{O}$ & 2.109408 & -1.780737 & 2.499729 \\
\hline $\mathrm{C}$ & 1.844607 & -1.657425 & -1.044412 \\
\hline $\mathrm{O}$ & 2.09964 & -2.53297 & -1.751906 \\
\hline $\mathrm{C}$ & -1.383119 & -1.641571 & 0.439779 \\
\hline $\mathrm{O}$ & -1.429837 & -2.787484 & 0.639166 \\
\hline $\mathrm{C}$ & -2.870773 & 0.574036 & 1.124272 \\
\hline $\mathrm{O}$ & -3.703615 & 0.809046 & 1.904165 \\
\hline $\mathrm{C}$ & -2.788575 & -0.224597 & -1.372152 \\
\hline $\mathrm{O}$ & -3.413171 & -1.184248 & -1.798914 \\
\hline
\end{tabular}


Species $\mathbf{2} \boldsymbol{\mu} \mathbf{H S H}^{*}$

Symbol

$\mathrm{Fe}$

$\mathrm{Fe}$

$\mathrm{S}$

S

C

$\mathrm{O}$

C

O

C

O

C

$\mathrm{O}$

C

$\mathrm{O}$

C

O

H

C

$\mathrm{H}$

H

C

H

H

H

Species $\mathbf{2} \boldsymbol{\mu} \mathbf{H t H}^{\mathrm{S}_{*}}$

Symbol

$\mathrm{Fe}$

$\mathrm{Fe}$

S

S

C

$\mathrm{O}$

C

Fe

$\begin{array}{rrr}0.147085 & 2.460878 & -1.203969 \\ 0.902697 & 2.966263 & -1.812414 \\ -0.832626 & 2.845992 & -1.501148 \\ 0.404388 & 2.716572 & 0.292901 \\ -0.284439 & 3.476127 & 0.675919 \\ 1.422151 & 3.082773 & 0.447896 \\ -2.921673 & 0.768987 & -1.917154\end{array}$

X

1.135882

$-1.379582$

0.082617

2.264807

2.387225

3.180726

1.649624

1.966373

$-0.225868$

$-0.442385$

$-2.050124$

$-2.544632$

$-2.592555$

$-3.370101$

$-2.102395$

$-2.539441$

$-0.101424$

0.61074

1.40929

$-0.227184$

1.093238

0.274261

1.581367

3.217479

$\begin{array}{r}Y \\ -0.461892 \\ -0.028434 \\ 1.024385 \\ 1.43021 \\ -0.955729 \\ -1.281586 \\ -1.590104 \\ -2.310094 \\ -1.598341 \\ -2.658129 \\ 1.49722 \\ 2.439566 \\ -0.333082 \\ -0.521053 \\ -1.12483 \\ -1.832088 \\ 0.094185 \\ 2.694941 \\ 2.995451 \\ 3.383591 \\ 2.775284 \\ 2.664332 \\ \hline .733968 \\ \hline\end{array}$

Y

0.008561

0.071901

$-1.448369$

1.028701

$-1.148224$

$-1.917589$

1.289632

2.129666

$-0.591499$

$-1.073869$

0.790615

1.229177

$-1.209228$

$-2.035787$

1.27454

2.069583

1.078656

$-0.863719$

$-1.548216$

$-1.008898$

0.577

1.288399

0.770186

0.118389

X

1.208296

$-1.561227$

$-0.079296$

1.823694

2.768915

3.749839

1.866909
Y

$-0.439565$

$-0.010285$

0.829317

1.535967

$-0.004403$

0.254846

$-1.308008$

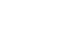




$\begin{array}{lrrr}\mathrm{O} & 2.251442 & -1.841737 & 2.378593 \\ \mathrm{C} & 0.940095 & -1.936303 & -0.975548 \\ \mathrm{O} & 0.803459 & -2.890382 & -1.593475 \\ \mathrm{C} & -2.857122 & 1.133645 & -0.26294 \\ \mathrm{O} & -3.651678 & 1.927241 & -0.503314 \\ \mathrm{C} & -2.077732 & -1.398493 & -0.934229 \\ \mathrm{O} & -2.476146 & -2.253159 & -1.585858 \\ \mathrm{C} & -2.28096 & -0.62392 & 1.665672 \\ \mathrm{O} & -2.696041 & -0.98118 & 2.674765 \\ \mathrm{H} & -0.228742 & -0.703539 & 0.827288 \\ \mathrm{C} & 0.433999 & 2.554986 & -0.991263 \\ \mathrm{H} & 1.337389 & 2.727433 & -1.583304 \\ \mathrm{H} & -0.35271 & 3.217853 & -1.366258 \\ \mathrm{C} & 0.693252 & 2.787642 & 0.483751 \\ \mathrm{H} & -0.247917 & 2.793812 & 1.037824 \\ \mathrm{H} & 1.174463 & 3.762602 & 0.618193 \\ \mathrm{H} & -1.187798 & 1.097512 & 1.086435\end{array}$

Species $\mathbf{2} \boldsymbol{\mu} \mathbf{H t H}^{\mathrm{a} *}$

Symbol

$\mathrm{Fe}$

$\mathrm{Fe}$

$\mathrm{S}$

S

C

$\mathrm{O}$

C

$\mathrm{O}$

C

O

C

O

C

O

C

O

$\mathrm{H}$

C

$\mathrm{H}$

$\mathrm{H}$

C

$\mathrm{H}$

$\mathrm{H}$

$\begin{array}{lrrr}\mathrm{O} & 2.251442 & -1.841737 & 2.378593 \\ \mathrm{C} & 0.940095 & -1.936303 & -0.975548 \\ \mathrm{O} & 0.803459 & -2.890382 & -1.593475 \\ \mathrm{C} & -2.857122 & 1.133645 & -0.26294 \\ \mathrm{O} & -3.651678 & 1.927241 & -0.503314 \\ \mathrm{C} & -2.077732 & -1.398493 & -0.934229 \\ \mathrm{O} & -2.476146 & -2.253159 & -1.585858 \\ \mathrm{C} & -2.28096 & -0.62392 & 1.665672 \\ \mathrm{O} & -2.696041 & -0.98118 & 2.674765 \\ \mathrm{H} & -0.228742 & -0.703539 & 0.827288 \\ \mathrm{C} & 0.433999 & 2.554986 & -0.991263 \\ \mathrm{H} & 1.337389 & 2.727433 & -1.583304 \\ \mathrm{H} & -0.35271 & 3.217853 & -1.366258 \\ \mathrm{C} & 0.693252 & 2.787642 & 0.483751 \\ \mathrm{H} & -0.247917 & 2.793812 & 1.037824 \\ \mathrm{H} & 1.174463 & 3.762602 & 0.618193 \\ \mathrm{H} & -1.187798 & 1.097512 & 1.086435\end{array}$

X

1.189591

$-1.572586$

$-0.048496$

1.725086

2.775351

3.775229

1.813598

2.17768

0.934884

0.822584

$-1.769368$

$-1.997973$

$-2.96515$

$-3.83521$

$-2.369209$

$-2.840487$

$-0.2515$

0.497262

1.420543

$-0.258348$

0.723062

$-0.228969$

1.273766
Y

$-0.49746$

$-0.179401$

0.842801

1.407383

$-0.023948$

0.270135

$-1.460904$

$-2.056271$

$-1.935164$

$-2.848516$

0.964282

1.581962

0.384847

0.72371

$-1.5689$

$-2.515372$

$-0.784491$

2.542805

2.701268

3.236609

2.745708

2.841193

3.679149
Z

$-0.071002$

$-0.046066$

$-1.456646$

1.281227

$-0.787819$

$-1.26548$

1.321173

2.22872

$-1.149578$

$-1.829692$

1.376978

2.315111

$-1.014537$

$-1.680934$

0.72836

1.175337

0.723677

$-0.983557$

$-1.548562$

$-1.366442$

0.50142

1.022939

0.658939 
Species $\mathbf{2} \boldsymbol{\mu} \mathbf{H H}^{\mathrm{Co}}$

Symbol

$\mathrm{Fe}$

$\mathrm{Fe}$

S

S

C

O

C

O

C

O

C

O

C

O

C

O

C

$\mathrm{H}$

$\mathrm{H}$

C

$\mathrm{H}$

$\mathrm{H}$

$\mathrm{H}$

$\mathrm{H}$

Species $\mathbf{2} \boldsymbol{\mu} \mathbf{H S H}^{\mathrm{in} *}$ Symbol

$\mathrm{Fe}$

$\mathrm{Fe}$

S

S

C

O

C

O

C

O

C

O

C

\section{X}

$-1.286125$

1.281167

$-0.131414$

$-0.228546$

$-2.810331$

$-3.767782$

$-1.77964$

$-2.056318$

$-1.744158$

$-2.008837$

2.061612

2.553552

2.636816

3.515634

2.03569

2.898617

$-0.36666$

$-1.249015$

0.502831

$-0.506552$

0.224565

$-1.500574$

0.097418

1.573441

\begin{tabular}{rr}
$Y$ & $Z$ \\
-0.388581 & 0.040226 \\
-0.059752 & -0.01686 \\
1.068822 & 1.44271 \\
0.923738 & -1.58659 \\
0.582459 & 0.014731 \\
1.208722 & -0.003102 \\
-1.574253 & -1.234689 \\
-2.323392 & -2.052984 \\
-1.434825 & 1.446831 \\
-2.09714 & 2.340343 \\
-1.109975 & -1.234762 \\
-1.79354 & -2.01602 \\
1.0694 & 0.088517 \\
1.810073 & 0.178024 \\
-1.104425 & 1.427589 \\
-1.959841 & 1.372461 \\
2.703968 & 0.619071 \\
3.166643 & 1.067288 \\
3.301227 & 0.904124 \\
2.616968 & -0.908618 \\
3.260608 & -1.404598 \\
2.938453 & -1.227581 \\
-1.258801 & 0.064245 \\
-0.887404 & 2.429306 \\
& \\
& \\
\hline
\end{tabular}

$-0.887404$

2.429306
1.142841

$-1.376901$

0.07125

2.330837

2.393705

3.182069

1.672305

1.995716

$-0.211719$

$-0.422683$

$-2.071664$

$-2.590335$

$-2.591996$
Y

$-0.452385$

$-0.037217$

1.016392

1.484341

$-0.960339$

$-1.296377$

$-1.546573$

$-2.24286$

$-1.606342$

$-2.675649$

1.486063

2.421908

$-0.370608$

1 


$\begin{array}{rrr}-3.370939 & -0.578045 & -2.019128 \\ -2.079112 & -1.127602 & 1.293175 \\ -2.501759 & -1.830513 & 2.099955 \\ -0.092913 & 0.105568 & 1.070017 \\ 0.580485 & 2.696489 & -0.875003 \\ 1.379632 & 3.006712 & -1.554342 \\ -0.263878 & 3.375375 & -1.023145 \\ 1.04241 & 2.774023 & 0.571518 \\ 0.223068 & 2.591301 & 1.264874 \\ 1.478049 & 3.754042 & 0.781069 \\ 2.185182 & 1.426526 & 2.218092\end{array}$

Species $2 \mathbf{t H}_{2}$ *

$\begin{array}{rrr}X & Y & Z \\ -1.062309 & -0.491165 & -0.199783 \\ 1.526385 & 0.087072 & -0.020711 \\ -0.210579 & 1.284067 & 1.009884 \\ -3.000646 & 0.779626 & -0.676652 \\ -1.900135 & -1.032134 & 1.302017 \\ -2.481183 & -1.343559 & 2.238788 \\ -1.55055 & -1.968787 & -1.130488 \\ -1.875997 & -2.903986 & -1.706473 \\ 0.710923 & -1.45432 & 0.648661 \\ 0.771487 & -2.49487 & 1.180685 \\ 2.612249 & -0.977909 & -0.969047 \\ 3.30843 & -1.652217 & -1.584158 \\ 1.8431 & 1.475032 & -1.179781 \\ 2.117068 & 2.368062 & -1.848465 \\ 2.672018 & 0.36863 & 1.31393 \\ 3.409957 & 0.566989 & 2.172499 \\ -0.161047 & 0.075732 & -1.552929 \\ -0.939902 & 0.296755 & -1.675985 \\ -2.48624 & 2.425984 & 0.021968 \\ -2.971345 & 3.196143 & -0.586231 \\ -2.867034 & 2.511887 & 1.043402 \\ -0.976462 & 2.640721 & 0.006022 \\ -0.710575 & 3.589435 & 0.482007 \\ -0.584002 & 2.630727 & -1.011839\end{array}$




\section{Species $\mathbf{2} \boldsymbol{\mu} \mathbf{H t H}^{\mathrm{S}} \mathbf{S H}^{*+}$}

$\begin{array}{rrr}1.818136 & 1.605421 & 1.150544 \\ 2.831878 & -0.468853 & -0.722716 \\ 3.89237 & -0.573001 & -1.157776 \\ 1.256411 & -1.322237 & 1.49243 \\ 1.336734 & -1.894883 & 2.490373 \\ 0.651404 & -1.871685 & -0.922707 \\ 0.396449 & -2.835062 & -1.503135 \\ -2.745682 & 1.215373 & 0.119378 \\ -3.578292 & 2.012038 & 0.190741 \\ -2.16442 & -1.108291 & -1.130409 \\ -2.713643 & -1.817914 & -1.852237 \\ -1.77087 & -0.892069 & 1.529458 \\ -1.975958 & -1.454091 & 2.517901 \\ -0.867381 & 0.960545 & 1.115199 \\ 0.583307 & 2.615503 & -1.108672 \\ 1.524408 & 2.732727 & -1.653434 \\ -0.139601 & 3.318148 & -1.534063 \\ 0.760254 & 2.911077 & 0.36692 \\ -0.196969 & 2.908939 & 0.88594 \\ 1.259684 & 3.870949 & 0.518728 \\ 1.155677 & 1.529859 & 2.32049\end{array}$

$-1.238842$

1.521678

0.07259

$-1.417807$

$-2.804673$

$-3.774058$

$-1.947651$

$-2.357029$

$-1.014177$

$-0.874577$

2.882145

3.718588

2.027348

2.425923

2.247088

2.667707

0.155271

$-0.411646$

$-1.356737$

0.343178

$\begin{array}{rr}Y & Z \\ -0.387894 & -0.034808 \\ -0.050981 & -0.138232 \\ 0.797095 & 1.445926 \\ 1.605042 & -1.363317 \\ 0.015883 & 0.820626 \\ 0.268343 & 1.363879 \\ -1.32268 & -1.443193 \\ -1.902041 & -2.33423 \\ -1.89889 & 0.952961 \\ -2.847475 & 1.568107 \\ 1.027889 & 0.276186 \\ 1.772177 & 0.515116 \\ -1.461412 & 0.954281 \\ -2.317068 & 1.598292 \\ -0.694116 & -1.646661 \\ -1.062825 & -2.644936 \\ -0.744533 & -0.814703 \\ 2.543467 & 1.095079 \\ 2.695385 & 1.621956 \\ 3.179839 & 1.567001\end{array}$


Species $\mathbf{2} \boldsymbol{\mu} \mathbf{H S H}_{2}{ }^{*+}$

Symbol

$\mathrm{Fe}$

$\mathrm{Fe}$

S

S

C

$\mathrm{O}$

C

$\mathrm{O}$

C

$\mathrm{O}$

C

O

C

$\mathrm{O}$

C

O

H

C

$\mathrm{H}$

H

C

$\mathrm{H}$

H

$\mathrm{H}$

$\mathrm{H}$

Species 2(tH) ${ }_{2} \mathbf{S H}^{+}$

Symbol

$\mathrm{Fe}$

$\mathrm{Fe}$

$\mathrm{S}$

$\mathrm{S}$

C

$\mathrm{O}$

C

$\mathrm{O}$

$\begin{array}{rrr}-0.532309 & 2.896341 & -0.373339 \\ 0.44184 & 2.98766 & -0.850849 \\ -1.079435 & 3.831227 & -0.515759 \\ -2.674957 & 2.021756 & -1.106169 \\ 1.211423 & 1.077436 & -1.069643\end{array}$

1.143869

$-1.364543$

0.096041

2.175859

2.377288

3.136599

1.632793

1.922476

$-0.261763$

$-0.408037$

$-2.057495$

$-2.539184$

$-2.611008$

$-3.395864$

$-2.121791$

$-2.578342$

$-0.106106$

0.584078

1.364124

$-0.273921$

1.059407

0.257795

1.615221

3.365083

2.454672

\begin{tabular}{|c|c|}
\hline Y & $\mathrm{Z}$ \\
\hline-0.46882 & 0.028622 \\
\hline-0.049757 & 0.0676 \\
\hline 1.020398 & -1.448703 \\
\hline 1.445449 & 0.942008 \\
\hline-1.003373 & -1.150189 \\
\hline-1.366024 & -1.931083 \\
\hline-1.666972 & 1.273177 \\
\hline-2.435851 & 2.07346 \\
\hline-1.584169 & -0.595022 \\
\hline-2.642738 & -1.094503 \\
\hline 1.475644 & 0.798074 \\
\hline 2.415031 & 1.246111 \\
\hline-0.311332 & -1.210158 \\
\hline-0.473522 & -2.028096 \\
\hline-1.146493 & 1.269401 \\
\hline-1.847047 & 2.052329 \\
\hline 0.070793 & 1.101817 \\
\hline 2.72222 & -0.912387 \\
\hline 3.033949 & -1.610586 \\
\hline 3.385025 & -1.053732 \\
\hline 2.875555 & 0.523404 \\
\hline 2.784011 & 1.256396 \\
\hline 3.798869 & 0.694758 \\
\hline 1.870904 & 0.453262 \\
\hline 1.720641 & 987 \\
\hline
\end{tabular}

1.720641

2.239873

$\begin{array}{rrr}X & Y & Z \\ -1.742393 & -0.309834 & 0.042491 \\ 1.742248 & -0.31053 & 0.04244 \\ 0.000202 & 0.620155 & 1.338378 \\ 0.000287 & 0.513432 & -1.417909 \\ -2.857852 & 1.182915 & -0.090928 \\ -3.617172 & 2.028984 & -0.197049 \\ -2.489262 & -1.23412 & -1.290416 \\ -2.90189 & -1.863366 & -2.151305\end{array}$




$\begin{array}{rrr}-2.676302 & -1.132554 & 1.343984 \\ -3.215401 & -1.693861 & 2.180994 \\ 2.488152 & -1.233478 & -1.291888 \\ 2.899751 & -1.861462 & -2.154057 \\ 2.858017 & 1.182065 & -0.089509 \\ 3.617087 & 2.02854 & -0.194529 \\ 2.676451 & -1.134031 & 1.34319 \\ 3.21597 & -1.695462 & 2.179796 \\ -0.000398 & 2.363501 & 0.73296 \\ -0.889931 & 2.855759 & 1.127949 \\ 0.886767 & 2.857798 & 1.130721 \\ 0.002051 & 2.270296 & -0.811274 \\ 0.885037 & 2.75529 & -1.228863 \\ -0.877537 & 2.758761 & -1.231966 \\ -0.955622 & -1.579966 & 0.099358 \\ 0.955048 & -1.580397 & 0.098208 \\ 0.000273 & 0.790279 & 2.681369\end{array}$

Species $\mathbf{2} \boldsymbol{\mu} \mathbf{H t H S H}^{\#+}$
Symbol

$\mathrm{Fe}$

$\mathrm{Fe}$

S

C

$\mathrm{O}$

C

O

C

O

C

O

C

O

C

O

C

$\mathrm{H}$

$\mathrm{H}$

C

H

$\mathrm{S}$

$\mathrm{H}$

H

$\mathrm{H}$

Fe

e

C

O

(

(

(

(

C

O

O

C

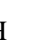

C

$\mathrm{H}$

S

$\mathrm{H}$

H

C
C
C
C
H
C
H
H
H

$\mathrm{X}$

0.151634

1.201444

$-0.487327$

$-1.141376$

$-1.9164$

0.771568

1.154533

1.403438

2.200517

0.316221

$-0.147574$

1.361199

1.474053

2.839996

3.904934

$-2.253515$

$-2.846914$

$-2.302777$

$-2.777498$

$-2.194269$

$-4.54134$

$-4.329463$

$-2.78572$

2.016207

$\begin{array}{rr}\mathrm{Y} & \mathrm{Z} \\ -1.422535 & -0.10358 \\ 1.060102 & 0.065037 \\ 0.216759 & 1.365208 \\ -2.611672 & 0.509714 \\ -3.342298 & 0.904704 \\ -2.438153 & -1.53609 \\ -3.025541 & -2.429699 \\ -2.06312 & 1.015235 \\ -2.456369 & 1.724859 \\ 1.826964 & -1.362765 \\ 2.34711 & -2.269241 \\ 2.538387 & 1.06863 \\ 3.447018 & 1.753155 \\ 1.166426 & -0.662857 \\ 1.185727 & -1.078737 \\ 0.706355 & 1.008272 \\ 0.100282 & 1.696913 \\ 1.743867 & 1.350163 \\ 0.573614 & -0.417208 \\ 1.151761 & -1.131633 \\ 1.093411 & -0.540497 \\ 2.418438 & -0.41335 \\ -0.468901 & -0.747763 \\ 0.472672 & 1.190382 \\ & \end{array}$


Species $\mathbf{2} \boldsymbol{\mu} \mathbf{H t H}^{\mathrm{a}} \mathbf{S} \mathbf{H}^{*+}$

Symbol

$\mathrm{Fe}$

$\mathrm{Fe}$

S

S

C

O

C

$\mathrm{O}$

C

O

C

O

C

O

C

O

C

$\mathrm{H}$

$\mathrm{H}$

C

$\mathrm{H}$

$\mathrm{H}$

$\mathrm{H}$

$\mathrm{H}$

H

Species $\mathbf{2} \boldsymbol{\mu} \mathbf{H}_{2} \mathbf{S H}^{\#+}$

Symbol

$\mathrm{Fe}$

$\mathrm{Fe}$

S

C

$\mathrm{O}$

C

O

C

O

C

O

C

\begin{tabular}{rrr}
$X$ & $Y$ & $Z$ \\
1.569183 & 0.168254 & -0.037293 \\
-1.171168 & 0.498261 & -0.035331 \\
-1.484403 & -1.378163 & 1.389145 \\
0.062581 & -0.928087 & -1.397752 \\
1.815955 & -1.089564 & 1.290209 \\
2.051615 & -1.843915 & 2.116736 \\
2.975124 & -0.293038 & -1.043567 \\
3.84855 & -0.557534 & -1.733742 \\
2.379658 & 1.523521 & 0.812002 \\
2.858841 & 2.434067 & 1.312738 \\
-0.808072 & 1.909779 & -1.142009 \\
-0.649482 & 2.810813 & -1.820743 \\
-2.7572 & 0.165919 & -0.886899 \\
-3.739143 & -0.042183 & -1.426567 \\
-1.819654 & 1.609296 & 1.262041 \\
-2.195971 & 2.296736 & 2.089129 \\
-1.523831 & -2.729514 & 0.121204 \\
-1.554473 & -3.680502 & 0.658182 \\
-2.435757 & -2.619582 & -0.465446 \\
-0.279228 & -2.638079 & -0.747316 \\
-0.415372 & -3.275374 & -1.624135 \\
0.609245 & -2.979016 & -0.216134 \\
1.470392 & 1.221874 & -1.107374 \\
-2.794144 & -1.391204 & 1.710748 \\
0.22908 & 0.760601 & 0.775699 \\
\hline
\end{tabular}

0.22908

0.760601

0.775699

$\begin{array}{rrr}X & Y & Z \\ 0.746056 & 1.199823 & 0.013556 \\ 0.755767 & -1.19584 & 0.019491 \\ -0.556959 & -0.007368 & -1.453912 \\ -0.481619 & 2.452835 & 0.524684 \\ -1.240808 & 3.238089 & 0.848031 \\ 1.973007 & 1.702799 & 1.277256 \\ 2.740609 & 1.983806 & 2.069406 \\ 1.398305 & 2.269166 & -1.322708 \\ 1.820296 & 2.889092 & -2.178486 \\ -0.461462 & -2.457922 & 0.532629 \\ -1.213235 & -3.25128 & 0.853896 \\ 1.418541 & -2.264905 & -1.311577\end{array}$


O

C

$\mathrm{O}$

$\mathrm{H}$

C

$\mathrm{H}$

$\mathrm{H}$

C

$\mathrm{H}$

$\mathrm{H}$

S

$\mathrm{H}$

$\mathrm{H}$

Species $2 \mathbf{t H}_{\mathbf{2}} \mathbf{t} \mathbf{H}^{+}$

Symbol

$\mathrm{Fe}$

$\mathrm{Fe}$

S

S

C

$\mathrm{O}$

C

O

C

$\mathrm{O}$

C

$\mathrm{O}$

C

$\mathrm{O}$

C

O

C

$\mathrm{H}$

$\mathrm{H}$

C

$\mathrm{H}$

$\mathrm{H}$

H

$\mathrm{H}$

$\mathrm{H}$

$\begin{array}{rrr}1.846661 & -2.88427 & -2.164718 \\ 1.986901 & -1.681945 & 1.286121 \\ 2.756734 & -1.952451 & 2.07974 \\ 1.831259 & 0.005944 & -0.421508 \\ -2.371864 & -0.007348 & -1.049503 \\ -2.757094 & 0.87809 & -1.561471 \\ -2.752518 & -0.885468 & -1.578505 \\ -2.81521 & -0.020762 & 0.407558 \\ -2.464803 & -0.901465 & 0.94333 \\ 0.234365 & 0.003308 & 1.094306 \\ -4.650492 & 0.066742 & 0.551338 \\ -4.917032 & -1.145937 & 0.025939 \\ -2.463986 & 0.856589 & 0.950128\end{array}$

$\mathrm{X}$

1.670856

$-1.718312$

0.005195

0.005255

2.642884

3.299016

2.687359

3.278531

2.688006

3.279642

$-2.620462$

$-3.135035$

$-2.771239$

$-3.518634$

$-2.621102$

$-3.136257$

$-0.00285$

0.861871

$-0.891587$

$-0.002373$

$-0.890575$

0.862976

1.175179

$-1.054881$

0.464222

$\begin{array}{rr}Y & Z \\ -0.401013 & -0.001201 \\ -0.329163 & -0.00102 \\ 0.499815 & -1.465416 \\ 0.490605 & 1.468145 \\ 1.143961 & 0.002538 \\ 2.073612 & 0.004477 \\ -1.057417 & 1.40357 \\ -1.452394 & 2.290052 \\ -1.049971 & -1.408957 \\ -1.440576 & -2.297062 \\ -1.130315 & 1.331759 \\ -1.682398 & 2.190855 \\ 1.202877 & 0.004423 \\ 2.067397 & 0.007248 \\ -1.120418 & -1.339105 \\ -1.666121 & -2.201921 \\ 2.212283 & -0.766209 \\ 2.744024 & -1.167269 \\ 2.702323 & -1.16838 \\ 2.2073 & 0.779875 \\ 2.695297 & 1.185734 \\ 2.735817 & 1.183857 \\ -2.036737 & -0.005049 \\ -1.676365 & -0.005879 \\ -1.672432 & -0.005473 \\ & \end{array}$

Species $\mathbf{2} \mathbf{t H}_{\mathbf{2}} \mathbf{S H}^{*+}$

Symbol

X

Y

Z 
$\mathrm{O}$

C

$\mathrm{O}$

C

O

C

$\mathrm{H}$

$\mathrm{H}$

C

$\mathrm{H}$

$\mathrm{H}$

$\mathrm{H}$

$\mathrm{H}$

$\mathrm{H}$

Species $\mathbf{2} \mathbf{t H}_{2} \mathbf{S H}^{\text {in } *+}$

Symbol

$\mathrm{Fe}$

$\mathrm{Fe}$

S

S

C

$\mathrm{O}$

C

O

C

$\mathrm{O}$

C

O

C

O

C

$\mathrm{O}$

$\begin{array}{rrr}-1.570107 & -0.120678 & -0.28835 \\ 1.069399 & -0.47973 & -0.124965 \\ 1.634307 & 1.299336 & 1.425091 \\ 0.040227 & 1.116567 & -1.40864 \\ -1.376451 & 0.644989 & 1.315808 \\ -1.367406 & 1.142146 & 2.349392 \\ -3.077183 & 0.823257 & -0.661668 \\ -4.023717 & 1.434518 & -0.855345 \\ -2.211269 & -1.6101 & 0.532352 \\ -2.618657 & -2.55012 & 1.041893 \\ 0.736182 & -1.804766 & -1.29298 \\ 0.620004 & -2.673922 & -2.03205 \\ 2.787962 & -0.411272 & -0.684101 \\ 3.870432 & -0.388883 & -1.054173 \\ 1.165589 & -1.668523 & 1.244226 \\ 1.230092 & -2.40388 & 2.119069 \\ 1.68697 & 2.711492 & 0.226638 \\ 1.806946 & 3.63096 & 0.804458 \\ 2.549791 & 2.574687 & -0.425543 \\ 0.389348 & 2.733433 & -0.56217 \\ 0.468471 & 3.479019 & -1.356524 \\ -0.464202 & 2.997001 & 0.063399 \\ -1.9827 & -0.684734 & -1.854381 \\ 2.961488 & 1.147769 & 1.60279 \\ -1.406801 & -1.178478 & -1.610136 \\ & & \\ & & \end{array}$

X

$-1.602998$

1.019622

2.06931

0.064187

$-1.071394$

$-0.949215$

$-2.922613$

$-3.737273$

$-2.546892$

$-3.144004$

0.341083

0.063471

2.627726

3.617628

1.295233

1.478287
Y

$-0.027847$

$-0.504334$

1.194352

1.180372

0.309707

0.577269

1.214255

2.016129

$-1.45592$

$-2.35328$

$-1.820535$

$-2.73128$

$-0.626843$

$-0.741378$

$-1.694267$

$-2.421448$
Z $-0.308697$ $-0.112613$ 1.269182 $-1.365524$

1.355692

2.472773

$-0.361222$

$-0.336836$

0.321321

0.700513

$-1.131607$

$-1.77576$

$-0.949156$

$-1.507903$

1.239245

2.101828 
Species $\mathbf{2} \mathbf{t H}_{\mathbf{2}} \boldsymbol{\mu} \mathbf{H}^{*+}$

Symbol

$\mathrm{Fe}$

$\mathrm{Fe}$

$\mathrm{S}$

S

C

$\mathrm{O}$

C

O

C

$\mathrm{O}$

C

$\mathrm{O}$

C

$\mathrm{O}$

C

$\mathrm{O}$

C

$\mathrm{H}$

$\mathrm{H}$

C

$\mathrm{H}$

H

H

$\mathrm{H}$

H

Species $\mathbf{2} \boldsymbol{\mu} \mathbf{H t H}^{\mathrm{S}} \mathbf{S H}^{\mathrm{in}{ }^{+}}$

Symbol

$\mathrm{Fe}$

$\mathrm{Fe}$

$\mathrm{S}$

S

$\begin{array}{rrr}1.846216 & 2.681971 & 0.201183 \\ 2.033015 & 3.561548 & 0.822289 \\ 2.642984 & 2.609269 & -0.541875 \\ 0.4749 & 2.738157 & -0.444184 \\ 0.457077 & 3.538072 & -1.189345 \\ -0.308398 & 2.938647 & 0.287468 \\ -2.211752 & -0.299051 & -1.907602 \\ 1.178224 & 1.504811 & 2.231859 \\ -1.656052 & -0.859406 & -1.831802\end{array}$

$\mathrm{X}$

1.398093

$-1.298355$

$-0.51487$

$-0.005849$

1.465423

2.270571

2.937091

3.887206

2.199347

2.677293

$-1.727165$

$-1.985372$

$-2.812936$

$-3.759816$

$-1.986477$

$-2.389091$

$-0.51649$

$-0.003714$

$-1.562679$

0.145767

$-0.315036$

1.212952

1.392906

0.023878

1.156315

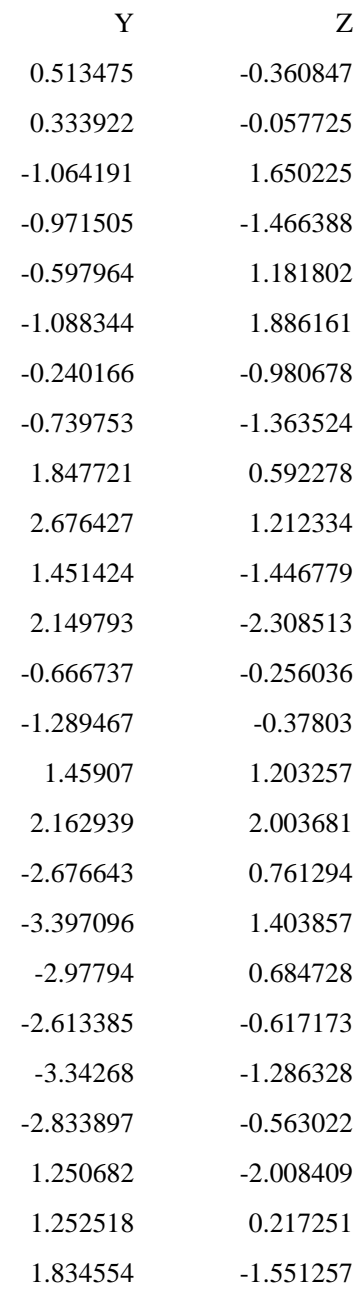

1.834554

$-1.551257$

$\begin{array}{rrr}\mathrm{X} & \mathrm{Y} & \mathrm{Z} \\ 1.236919 & -0.386374 & 0.033392 \\ -1.533413 & -0.037961 & 0.140455 \\ -0.079614 & 0.800357 & -1.439139 \\ 1.620893 & 1.648017 & 1.264559\end{array}$


C
O
O
C
O
C
O
C
O
C
O
H
C
H
H
C
H
H
H
H

O

O

C

C

O

H

C

H

H

Species $\mathbf{2} \boldsymbol{\mu} \mathbf{H t H}_{\mathbf{2}}{ }^{\text {*+ }}$

Symbol

$\mathrm{Fe}$

$\mathrm{Fe}$

$\mathrm{S}$

S

C

O

C

$\mathrm{O}$

C

O

C

O

C

$\mathrm{O}$

C

O

$\mathrm{H}$

C

H

H

$\begin{array}{rrr}2.80844 & -0.035087 & -0.848566 \\ 3.777984 & 0.178014 & -1.406719 \\ 1.940613 & -1.312942 & 1.447577 \\ 2.34417 & -1.886536 & 2.345372 \\ 0.978865 & -1.906772 & -0.932521 \\ 0.827967 & -2.861245 & -1.535931 \\ -2.897346 & 1.029644 & -0.28974 \\ -3.736023 & 1.768 & -0.539446 \\ -2.036961 & -1.461684 & -0.936379 \\ -2.440938 & -2.323084 & -1.569471 \\ -2.256027 & -0.668222 & 1.655468 \\ -2.673414 & -1.02658 & 2.659143 \\ -0.15445 & -0.697862 & 0.833813 \\ 0.412668 & 2.544283 & -1.091845 \\ 1.339927 & 2.701075 & -1.648763 \\ -0.353973 & 3.184755 & -1.537843 \\ 0.573425 & 2.899056 & 0.372979 \\ -0.385462 & 2.93959 & 0.885893 \\ 1.083189 & 3.858925 & 0.481524 \\ 0.811266 & 1.447944 & 2.321087 \\ -1.232135 & 1.106109 & 1.052097\end{array}$

$\begin{array}{rrr}X & Y & Z \\ 1.210032 & -0.430498 & -0.019424 \\ -1.523018 & -0.011026 & 0.223708 \\ -0.055833 & 0.899769 & -1.369356 \\ 1.515049 & 1.431743 & 1.456497 \\ 2.813749 & 0.14804 & -0.628814 \\ 3.818694 & 0.514595 & -1.027977 \\ 1.878789 & -1.444485 & 1.34649 \\ 2.26883 & -2.064688 & 2.219485 \\ 1.125379 & -1.835338 & -1.190033 \\ 1.108571 & -2.705096 & -1.926913 \\ -2.888196 & 1.151621 & -0.212643 \\ -3.712677 & 1.887418 & -0.47982 \\ -2.020208 & -1.213725 & -1.068089 \\ -2.324172 & -1.969449 & -1.860617 \\ -2.381552 & -0.970921 & 1.538626 \\ -2.883565 & -1.574271 & 2.360791 \\ -0.286045 & -0.95702 & 0.64247 \\ 0.50517 & 2.602848 & -0.921149 \\ 1.439903 & 2.715975 & -1.477497 \\ -0.227091 & 3.29911 & -1.340174\end{array}$




$\begin{array}{rrr}0.705297 & 2.839645 & 0.563331 \\ -0.251927 & 3.049034 & 1.049108 \\ 1.343317 & 3.715386 & 0.706641 \\ -0.54047 & 0.863532 & 1.422902 \\ -1.323499 & 1.115261 & 1.445015\end{array}$

Species 3

Species $\mathbf{3} \boldsymbol{\mu} \mathbf{H}^{+}$

$\begin{array}{rrr}X & Y & Z \\ 0.732405 & 1.224883 & -0.000524 \\ 0.732301 & -1.22453 & 0.000544 \\ -0.55159 & -0.000328 & -1.517562 \\ -0.551564 & 0.000758 & 1.517569 \\ -2.151852 & -0.000042 & -0.700036 \\ -4.56216 & 0.000327 & 0.696878 \\ -2.151841 & 0.00044 & 0.700075 \\ -3.351914 & -0.000311 & -1.405445 \\ -4.562171 & -0.000116 & -0.6968 \\ -3.351892 & 0.000611 & 1.405503 \\ -3.348511 & -0.000669 & -2.490909 \\ -5.500828 & -0.000323 & -1.241923 \\ -3.348471 & 0.000944 & 2.490967 \\ -5.500808 & 0.000448 & 1.242016 \\ -0.168792 & 2.776146 & -0.000903 \\ -0.742423 & 3.771448 & -0.000979 \\ 1.924565 & 1.575772 & 1.288952 \\ 2.681045 & 1.80155 & 2.125324 \\ 1.925122 & 1.574113 & -1.289919 \\ 2.682183 & 1.798888 & -2.126044 \\ 1.924524 & -1.575224 & 1.29004 \\ 2.681121 & -1.801079 & 2.126282 \\ -0.170186 & -2.775062 & 0.000615 \\ -0.744874 & -3.769741 & 0.000398 \\ 1.924204 & -1.576468 & -1.288926 \\ 2.680585 & -1.803263 & -2.125104 \\ & & \end{array}$

X 0.853901

0.748009

$-0.434811$

$-0.434222$

$-2.034932$

$-4.439477$

$-2.034595$
Y $-1.255172$ 1.303369

$-0.027393$

$-0.029682$

$-0.074749$

$-0.116234$

$-0.078174$

\section{t}




$\begin{array}{rrr}-3.231261 & -0.091299 & 1.408855 \\ -4.439834 & -0.110509 & 0.696184 \\ -3.230584 & -0.101002 & -1.409089 \\ -3.228906 & -0.086178 & 2.49375 \\ -5.378343 & -0.119373 & 1.240384 \\ -3.227627 & -0.103722 & -2.493972 \\ -5.377673 & -0.130472 & -1.241436 \\ -0.237136 & -2.712675 & -0.001052 \\ -0.933535 & -3.614163 & -0.003873 \\ 1.948981 & -1.862263 & -1.336119 \\ 2.630093 & -2.217538 & -2.177016 \\ 1.945015 & -1.86553 & 1.339761 \\ 2.621775 & -2.227463 & 2.181224 \\ 1.785419 & 1.998231 & -1.337856 \\ 2.43237 & 2.407421 & -2.18132 \\ -0.464811 & 2.664199 & -0.002738 \\ -1.237008 & 3.501727 & -0.006346 \\ 1.782797 & 2.001431 & 1.339085 \\ 2.427243 & 2.415311 & 2.182243 \\ 1.842416 & 0.068919 & -0.003577\end{array}$

Species $\mathbf{3} \boldsymbol{\mu H}$

Symbol

X

Y

0.834553

$-1.290032$

0.834087

1.290238

$-0.533139$

$-0.000137$

$-0.53323$

$-0.000153$

$-2.098775$

$-0.000352$

$-4.515868$

$-0.000473$

$-2.098806$

$-0.000357$

$-3.311402$

$-0.000404$

$-4.515833$

$-0.000463$

$-0.003577$

1.888449

1.896212

$-1.373434$ 
O

C

O

C

O

H

Species $\mathbf{3} \boldsymbol{\mu} \mathbf{H}^{*}$

Symbol

$\mathrm{Fe}$

$\mathrm{Fe}$

S

S

C

C

C

C

C

C

$\mathrm{H}$

$\mathrm{H}$

$\mathrm{H}$

$\mathrm{H}$

C

$\mathrm{O}$

C

O

C

O

C

O

C

O

C

O

$\mathrm{H}$

Species $\mathbf{3} \boldsymbol{\mu} \mathbf{H}^{*-}$

Symbol

$\mathrm{Fe}$

$\mathrm{Fe}$

S

S

C

$\begin{array}{rrr}2.58687 & 2.258819 & -2.207737 \\ -0.284559 & 2.695694 & 0.000046 \\ -1.017537 & 3.577548 & 0.000087 \\ 1.888614 & 1.896145 & 1.373395 \\ 2.587203 & 2.258766 & 2.207551 \\ 1.861748 & 0.000304 & -0.000063\end{array}$

$\begin{array}{rrr}X & Y & Z \\ -0.715427 & 1.33636 & 0.14038 \\ -1.088312 & -1.342653 & -0.078231 \\ 0.404858 & -0.264647 & 1.347043 \\ 1.149856 & 1.513155 & -1.299687 \\ 2.040323 & -0.297113 & 0.624709 \\ 4.608384 & -0.416083 & -0.449001 \\ 2.341166 & 0.466599 & -0.516464 \\ 3.019343 & -1.09614 & 1.233946 \\ 4.301329 & -1.16181 & 0.695874 \\ 3.644755 & 0.391161 & -1.045005 \\ 2.767346 & -1.665187 & 2.123941 \\ 5.055506 & -1.78421 & 1.166735 \\ 3.893075 & 0.975149 & -1.926139 \\ 5.606969 & -0.45712 & -0.873895 \\ 0.080863 & 2.707359 & 1.013526 \\ 0.599632 & 3.566087 & 1.563244 \\ -1.608993 & 2.30976 & -1.094091 \\ -2.164639 & 2.89834 & -1.902108 \\ -2.146476 & 1.199545 & 1.249223 \\ -3.049907 & 1.141549 & 1.949515 \\ 0.038758 & -1.950904 & -1.431281 \\ 0.734062 & -2.310188 & -2.269112 \\ -1.121838 & -2.829977 & 0.945418 \\ -1.124582 & -3.782299 & 1.589827 \\ -2.656922 & -1.677741 & -0.894893 \\ -3.654111 & -1.872388 & -1.434517 \\ -1.268895 & 0.1221 & -0.830756\end{array}$

$0.1221 \quad-0.830756$

$\begin{array}{rrr}X & Y & Z \\ -0.664635 & -1.282811 & -0.09292 \\ -1.018729 & 1.240561 & 0.165257 \\ 0.538866 & 0.252192 & -1.336549 \\ 1.373713 & -1.874683 & 1.034547 \\ 2.169079 & 0.243576 & -0.603577\end{array}$




$\begin{array}{rrr}4.759666 & 0.284186 & 0.442181 \\ 2.508067 & -0.670838 & 0.419492 \\ 3.123743 & 1.144102 & -1.103039 \\ 4.41563 & 1.172348 & -0.585101 \\ 3.823272 & -0.619419 & 0.932508 \\ 2.840569 & 1.827213 & -1.899468 \\ 5.143642 & 1.877846 & -0.974184 \\ 4.099442 & -1.308614 & 1.725654 \\ 5.762974 & 0.29549 & 0.859734 \\ -0.625587 & -2.552855 & -1.312973 \\ -0.585761 & -3.381631 & -2.117536 \\ -1.592716 & -2.214968 & 1.092243 \\ -2.17787 & -2.812872 & 1.888118 \\ -2.131516 & -0.299827 & -0.70049 \\ -3.186279 & -0.339815 & -1.244927 \\ 0.237563 & 2.186066 & 1.061025 \\ 0.999522 & 2.835238 & 1.633088 \\ -1.571964 & 2.407924 & -1.063449 \\ -1.926176 & 3.169448 & -1.856573 \\ -2.34935 & 1.498605 & 1.299299 \\ -3.208983 & 1.64269 & 2.057725 \\ -0.59136 & -0.011451 & 1.091666\end{array}$

$\mathbf{T S}_{1}$

Symbol

X

Y

Z

$\mathrm{Fe}$

1.112147

$-0.549145$

$-0.002564$

$-1.368618$

$-0.019778$

0.079908

S

0.118888

0.987619

$-1.45476$

2.248756

1.531926

0.989095

2.386674

$-1.049902$

$-1.135754$

3.183565

$-1.387066$

$-1.895206$

1.614632

$-1.67238$

1.291326

1.928878

$-2.3806$

2.141412

$-0.279065$

$-1.623517$

$-0.583775$

$-0.507945$

$-2.679282$

$-1.067792$

$-1.992503$

1.52706

0.795363

$-2.436927$

2.486637

1.245863

$-2.610056$

$-0.263524$

$-1.197836$

$-3.395902$

$-0.416235$

$-2.021376$

$-2.121282$

$-1.090796$

1.291862

$-2.577025$

$-1.77885$

2.091356

$-0.111864$

1.08433

0.677497

0.056644

$-0.961632$

1.501556

2.686624

$-1.646208$ 
H

C

H

$\mathrm{H}$

$\mathrm{H}$

$\begin{array}{rrr}-0.146817 & 3.367985 & -1.191872 \\ 1.125003 & 2.894591 & 0.480668 \\ 0.279332 & 2.915576 & 1.167606 \\ 1.665014 & 3.839979 & 0.567708 \\ 3.236175 & 2.189452 & 1.622745\end{array}$

$\mathbf{T S}_{2}$

Symbol

$\mathrm{Fe}$

$\mathrm{Fe}$

S

S

C

O

C

O

C

O

C

O

C

O

C

O

$\mathrm{H}$

C

$\mathrm{H}$

$\mathrm{H}$

C

H

H

$\mathrm{H}$

$\mathrm{TS}_{3}$

Symbol

$\mathrm{Fe}$

$\mathrm{Fe}$

S

S

C

O

$\begin{array}{rrr}X & Y & Z \\ 1.147129 & -0.431572 & -0.062933 \\ -1.414327 & 0.038993 & 0.08792 \\ -0.009338 & 0.95315 & -1.482625 \\ 1.944846 & 1.57571 & 1.097382 \\ 2.732952 & -0.577888 & -0.891957 \\ 3.726466 & -0.751873 & -1.446237 \\ 1.421127 & -1.356052 & 1.446079 \\ 1.601127 & -1.931548 & 2.427566 \\ 0.404326 & -1.850858 & -0.82569 \\ 0.073295 & -2.83009 & -1.345959 \\ -2.611112 & 1.308699 & 0.337484 \\ -3.368671 & 2.155911 & 0.541501 \\ -2.301329 & -0.919025 & -1.177484 \\ -2.940042 & -1.498635 & -1.940773 \\ -1.768898 & -1.011485 & 1.464423 \\ -1.971487 & -1.655211 & 2.402128 \\ -0.691925 & 0.782098 & 1.170821 \\ 0.59438 & 2.642959 & -1.051866 \\ 1.516688 & 2.787174 & -1.621922 \\ -0.146149 & 3.355496 & -1.427246 \\ 0.818835 & 2.883712 & 0.427253 \\ -0.11633 & 2.829694 & 0.982795 \\ 1.294936 & 3.851829 & 0.600394 \\ 1.414612 & 1.510228 & 2.334153 \\ & & \\ & & \end{array}$

\section{X}

1.130199

$-1.428881$

0.025292

1.488096

2.839478

3.933622
Z

0.08792

482625

891957

446237

.446079

27566

345959

0.337484

541501

.940773

4423

402128

051866

$-1.621922$

0.427253

0.982795

2.334153

\section{Z}

$-0.072431$

0.135203

$-1.43044$

1.45703

$-0.596145$

$-0.919285$ 


$\begin{array}{rrr}1.294465 & -1.499019 & 1.357391 \\ 1.413749 & -2.175183 & 2.280692 \\ 0.796091 & -1.792787 & -1.171396 \\ 0.625038 & -2.676599 & -1.886971 \\ -2.88076 & 1.150779 & 0.108935 \\ -3.804993 & 1.834778 & 0.131156 \\ -2.033717 & -0.994888 & -1.155787 \\ -2.46583 & -1.687736 & -1.963862 \\ -1.743527 & -1.074679 & 1.436916 \\ -1.970267 & -1.815712 & 2.28948 \\ -1.082209 & 1.10649 & 1.344663 \\ 0.749562 & 2.655922 & -0.983721 \\ 1.719625 & 2.673562 & -1.488088 \\ 0.115796 & 3.423169 & -1.439503 \\ 0.900552 & 2.908023 & 0.505371 \\ -0.058594 & 3.200994 & 0.942077 \\ 1.614648 & 3.715705 & 0.682189 \\ -0.141174 & 0.996612 & 1.442098\end{array}$

$\mathbf{T S}_{2}$,

Symbol

$\mathrm{Fe}$

$X$
-1.048219

Y

Z

$\mathrm{Fe}$

1.472739

$-0.523146$

$-0.115199$

0.09355

$-0.051222$

$-0.187519$

1.233993

1.1652

$-2.737039$

0.948083

$-1.016535$

$-2.079895$

$-1.03284$

1.279377

$-1.3116$

2.145169

$-2.776564$

$-1.936117$

$-1.134958$

$-1.800612$

$-2.837896$

$-1.778502$

0.541493

$-1.4551$

0.660801

0.726084

$-2.472591$

1.223787

2.462129

$-1.003538$

$-1.069143$

3.084151

$-1.707449$

$-1.728292$

1.910715

1.564488

$-1.065869$

2.289371

2.487876

$-1.633935$

2.64882

0.164091

1.27717

3.409705

0.240117

2.135435

$-0.08483$

0.07202

$-1.327077$

$-0.968444$

0.423985

$-1.427034$

$-2.384845$

2.449274

0.0117

$-2.810562$

3.304764

$-0.522108$

$-2.905941$

2.346623

0.967594

$-0.897133$

2.674954

0.239279

$-0.734349$

3.558233

0.863973 
TS ${ }^{\prime}$

Symbol

$\mathrm{Fe}$

$\mathrm{Fe}$

S

S

C

O

C

$\mathrm{O}$

C

O

C

O

C

O

C

O

$\mathrm{H}$

$\mathrm{H}$

C

$\mathrm{H}$

$\mathrm{H}$

C

H

$\mathrm{H}$

$\mathrm{TS}_{4}$

Symbol

$\mathrm{Fe}$

$\mathrm{Fe}$

S

S

C

O

C

O

C

O

C

O

C
X

$-1.060937$

1.526267

$-0.254359$

$-3.049087$

$-1.800757$

$-2.312862$

$-1.351103$

$-1.553694$

1.065833

0.987042

2.378692

2.929555

1.569757

1.642494

2.892671

3.774292

$-0.387986$

$-1.100863$

$-2.604907$

$-3.15522$

$-2.954956$

$-1.108798$

$-0.876303$

$-0.751061$

X

1.205551

$-1.518047$

$-0.023428$

1.833443

2.73134

3.656824

1.951794

2.385194

0.830527

0.620966

$-2.850079$

$-3.660889$

$-2.052696$
Y

$-0.463135$

0.087695

1.289937

0.647082

$-1.143496$

$-1.567084$

$-1.978879$

$-2.952475$

$-1.300451$

$-2.19107$

$-1.089011$

$-1.83465$

1.310636

2.118832

0.734138

1.12298

0.185111

0.429111

2.324563

3.061782

2.394833

2.625092

3.578143

2.652326
$-0.189325$

0.029139

1.009041

$-0.566394$

1.256624

2.193604

$-1.149033$

$-1.72134$

1.10938

1.842596

$-1.011115$

$-1.690587$

$-1.315326$

$-2.129026$

1.005846

1.62972

$-2.242625$

$-2.18043$

0.111821

$-0.481733$

1.14542

0.042281

0.526731

$-0.987478$

\section{Y}

$-0.438065$

$-0.035588$

0.156804

0.892568

$-1.382283$

1.66186

0.941611

$-0.178403$

$-0.957533$

$-0.015233$

$-1.59831$

$-1.332378$

1.470656

$-1.867631$

2.378572

$-1.97157$

$-0.829198$

$-2.94733$

$-1.378214$

1.090607

$-0.220278$

1.87288

$-0.426581$

$-1.38167$ 


$\begin{array}{lll}-2.489851 & -2.177228 & -1.69683 \\ -2.288104 & -0.723478 & 1.621084 \\ -2.745661 & -1.122984 & 2.593392 \\ -0.161311 & -0.660332 & 0.920663 \\ 0.255374 & 2.639535 & -0.869483 \\ 1.101567 & 2.967725 & -1.474659 \\ -0.632685 & 3.189207 & -1.186023 \\ 0.533432 & 2.904025 & 0.612707 \\ -0.309219 & 2.781939 & 1.289455 \\ 0.966109 & 3.901593 & 0.709576 \\ 2.593151 & 1.989164 & 2.004277 \\ -1.195152 & 1.055015 & 1.123313\end{array}$

$\mathbf{T S}_{5}$

Symbol

$\begin{array}{rrr}X & Y & Z \\ 1.211675 & -0.428568 & -0.017929 \\ -1.51891 & -0.015502 & 0.21895 \\ -0.053971 & 0.895719 & -1.374708 \\ 1.480791 & 1.434143 & 1.465682 \\ 2.815551 & 0.15588 & -0.625225 \\ 3.819718 & 0.529689 & -1.018839 \\ 1.881951 & -1.440019 & 1.349385 \\ 2.272306 & -2.0587 & 2.223261 \\ 1.134871 & -1.834116 & -1.188423 \\ 1.119313 & -2.704535 & -1.924412 \\ -2.884492 & 1.145194 & -0.212982 \\ -3.709659 & 1.881869 & -0.476364 \\ -2.016964 & -1.221 & -1.071015 \\ -2.322938 & -1.978061 & -1.861654 \\ -2.373472 & -0.971764 & 1.53732 \\ -2.873479 & -1.571733 & 2.363414 \\ -0.277085 & -0.959622 & 0.641509 \\ 0.504717 & 2.60027 & -0.930509 \\ 1.442822 & 2.712088 & -1.481302 \\ -0.225125 & 3.294518 & -1.357023 \\ 0.694561 & 2.844841 & 0.554348 \\ -0.265523 & 3.062564 & 1.030911 \\ 1.338187 & 3.716081 & 0.698705 \\ -0.519748 & 0.875294 & 1.4255 \\ -1.316986 & 1.103156 & 1.438931\end{array}$

TS $_{6}$

Symbol

$\mathrm{Fe}$

$\begin{array}{rrr}X & Y & Z \\ 1.218296 & -0.411646 & -0.023982\end{array}$




$\begin{array}{rrr}-1.498949 & -0.011622 & 0.194734 \\ -0.048578 & 0.899555 & -1.387159 \\ 1.396383 & 1.408526 & 1.467248 \\ 2.82061 & 0.219933 & -0.574591 \\ 3.827428 & 0.621108 & -0.935168 \\ 1.879127 & -1.422041 & 1.346831 \\ 2.26318 & -2.040661 & 2.223999 \\ 1.199713 & -1.809664 & -1.204002 \\ 1.226226 & -2.677646 & -1.943501 \\ -2.955959 & 1.06468 & -0.292169 \\ -3.840849 & 1.700517 & -0.611795 \\ -1.930179 & -1.251833 & -1.03576 \\ -2.180497 & -2.042478 & -1.814537 \\ -2.341815 & -0.982346 & 1.537413 \\ -2.827282 & -1.602519 & 2.355777 \\ -0.260097 & -0.926533 & 0.63797 \\ 0.469017 & 2.616473 & -0.931801 \\ 1.39406 & 2.749336 & -1.499528 \\ -0.281443 & 3.298324 & -1.34191 \\ 0.682952 & 2.8497 & 0.553057 \\ -0.259203 & 3.117701 & 1.038227 \\ 1.371654 & 3.688353 & 0.686587 \\ -1.10139 & 1.272805 & 2.121748 \\ -1.817935 & 1.499679 & 2.043872 \\ & & \\ & & \end{array}$

$\mathbf{T S}_{7}$

$\begin{array}{lrrr}\text { Symbol } & \mathrm{X} & \mathrm{Y} & \mathrm{Z} \\ \mathrm{Fe} & -1.185424 & -0.451458 & -0.039619 \\ \mathrm{Fe} & 1.517737 & 0.12297 & -0.219287 \\ \mathrm{~S} & 0.015696 & 0.906692 & 1.395777 \\ \mathrm{~S} & -1.871697 & 1.500871 & -1.2514 \\ \mathrm{C} & -2.70581 & -0.415103 & 0.981417 \\ \mathrm{O} & -3.64649 & -0.39259 & 1.62437 \\ \mathrm{C} & -1.823156 & -1.448455 & -1.43379 \\ \mathrm{O} & -2.198694 & -2.055399 & -2.321858 \\ \mathrm{C} & -0.56047 & -1.929073 & 0.812923 \\ \mathrm{O} & -0.200422 & -2.871895 & 1.344616 \\ \mathrm{C} & 2.737668 & 1.330198 & -0.568913 \\ \mathrm{O} & 3.52792 & 2.128719 & -0.820201 \\ \mathrm{C} & 2.286424 & -0.692018 & 1.216924 \\ \mathrm{O} & 2.822653 & -1.089275 & 2.147232 \\ \mathrm{C} & 2.18823 & -1.26084 & -1.163459 \\ \mathrm{O} & 2.580181 & -2.214446 & -1.662906 \\ \mathrm{H} & 0.136424 & -0.367435 & -1.008225\end{array}$




$\begin{array}{lll}-0.648619 & 2.593433 & 1.041657 \\ -1.535851 & 2.685054 & 1.673064 \\ 0.098573 & 3.305815 & 1.403759 \\ -0.972564 & 2.880068 & -0.41134 \\ -0.07735 & 3.034013 & -1.014296 \\ -1.599367 & 3.769919 & -0.508877 \\ -3.094633 & 1.745942 & -0.73892 \\ 1.036658 & 1.456208 & -0.72753\end{array}$

TS $_{8}$

Symbol

$\mathrm{Fe}$

$-1.53599$

X

Y

Z

$\mathrm{Fe}$

1.18965

$-0.260062$

$-0.137554$

$\mathrm{S}$

1.157748

$-0.476151$

$-0.070425$

1.353625

1.484945

S

C

O

$-0.006455$

0.909545

$-1.480684$

$-1.839519$

1.079853

1.067147

$-2.156575$

1.900249

1.80162

$-2.941916$

0.038569

$-1.213506$

$-3.817131$

0.202782

$-1.932498$

$-2.308555$

$-1.439158$

0.993199

$-2.779638$

$-2.207553$

1.697359

1.311116

$-1.765195$

$-1.349184$

1.423336

$-2.557762$

$-2.162205$

2.905915

$-0.003279$

$-0.431039$

3.988294

0.279806

$-0.660728$

1.456066

$-1.648022$

1.303068

1.606993

$-2.367587$

2.176586

1.302802

2.739998

0.258189

1.207302

3.677026

0.811782

2.29665

2.684982

$-0.185966$

0.217381

2.625109

$-0.803814$

0.49607

3.251738

$-1.653971$

$-0.750058$

2.970879

$-0.439816$

$-1.323194$

$-1.441805$

$-1.086235$

2.415154

1.427004

1.970251

$-0.499699$

$-1.405747$

$-0.382168$

TS9

Symbol

$\begin{array}{rrr}X & Y & Z \\ -1.583141 & -0.042385 & -0.318581 \\ 0.996781 & -0.539908 & -0.097799 \\ 2.211115 & 1.280284 & 0.961195 \\ 0.097567 & 1.187929 & -1.370178 \\ -0.9224 & 0.220405 & 1.331405\end{array}$


O

C

O

C

O

C

O

C

O

C

O

C

$\mathrm{H}$

$\mathrm{H}$

C

$\mathrm{H}$

$\mathrm{H}$

$\mathrm{H}$

$\mathrm{H}$

$\mathrm{H}$

$\mathrm{TS}_{10}$

Symbol

$\mathrm{Fe}$

$\mathrm{Fe}$

S

S

C

O

C

O

C

O

C

O

C

O

C

O

C

$\mathrm{H}$

$\mathrm{H}$

C

$\mathrm{H}$

$\begin{array}{rrr}-0.777592 & 0.452126 & 2.45433 \\ -2.842338 & 1.261647 & -0.293451 \\ -3.61993 & 2.097203 & -0.228189 \\ -2.615547 & -1.395377 & 0.339554 \\ -3.269201 & -2.245286 & 0.732996 \\ 0.171851 & -1.85987 & -0.986692 \\ -0.143198 & -2.816445 & -1.546949 \\ 2.542364 & -0.756255 & -1.056954 \\ 3.482067 & -0.917856 & -1.682803 \\ 1.37362 & -1.690695 & 1.266425 \\ 1.622382 & -2.380889 & 2.141443 \\ 1.626407 & 2.916841 & 0.35296 \\ 1.539643 & 3.601045 & 1.198665 \\ 2.392255 & 3.279748 & -0.332831 \\ 0.278875 & 2.738737 & -0.33038 \\ 0.111064 & 3.562778 & -1.028235 \\ -0.523324 & 2.739826 & 0.404519 \\ -2.273607 & -0.278896 & -1.893334 \\ 2.730191 & 1.447439 & 2.190748 \\ -1.702995 & -0.827147 & -1.873731\end{array}$

X

$-1.54236$

0.976964

2.256154

0.074583

$-0.797162$

$-0.683406$

$-2.774819$

$-3.509838$

$-2.714124$

$-3.45821$

$-0.004473$

$-0.500739$

2.443575

3.336064

1.393642

1.668175

1.996648

2.264283

2.725153

0.578494

0.516891
Y

0.048292

$-0.531449$

1.040751

1.25406

0.066109

0.232504

1.35956

2.228583

$-1.245757$

$-2.058924$

$-1.704503$

$-2.513934$

$-0.720077$

$-0.859966$

$-1.830982$

$-2.630587$

2.590152

3.425241

2.534771

2.727616

3.588761
Z $-0.204902$

$-0.106325$

1.19492

$-1.303456$

1.388671

2.536177

$-0.163714$

$-0.038403$

0.321614

0.622924

$-1.067818$

$-1.716164$

$-1.180312$

$-1.876726$

1.11006

1.876427

0.225397

0.877398

$-0.58597$

$-0.293487$

$-0.96435$ 
H

H

$\mathrm{H}$

H

TS $_{11}$

Symbol

$\mathrm{Fe}$

$\mathrm{Fe}$

S

S

C

O

C

O

C

O

C

O

C

O

C

O

C

$\mathrm{H}$

$\mathrm{H}$

C

$\mathrm{H}$

$\mathrm{H}$

$\mathrm{H}$

$\mathrm{TS}_{12}$

Symbol

$\mathrm{Fe}$

$\mathrm{Fe}$

S

S

C

O

C

O

C

O

C

$\begin{array}{rrr}-0.135581 & 2.868046 & 0.517886 \\ -2.726835 & -0.097009 & -2.543119 \\ 1.435616 & 1.317633 & 2.228979 \\ -2.218067 & -0.426436 & -2.978717\end{array}$

X

$-1.407013$

1.104224

1.360918

$-0.050002$

$-2.033222$

$-2.415782$

$-3.002857$

$-3.963146$

$-1.795089$

$-2.032275$

0.800442

0.672879

2.794301

3.849204

1.586891

1.893262

1.358866

1.307582

2.314594

0.205921

0.426514

$-0.737323$

0.061867

$-0.193792$

$-0.425147$

1.274774

1.100329

1.017574

1.794364

$-0.048262$

0.026146

$-1.604044$

$-2.475792$

$-1.739078$

$-2.576838$

$-0.046521$

0.228386

$-1.645707$

$-2.398272$

2.710478

3.603672

2.715724

2.73266

3.4444

3.026498

0.253888

$-0.100849$

$-0.135186$

1.554299

$-1.449844$

1.059066

1.801141

$-1.048115$

$-1.653328$

1.012167

1.707143

$-1.348515$

$-2.116347$

$-0.726722$

$-1.063358$

1.125286

1.927106

0.401744

1.032838

$-0.126162$

$-0.593977$

$-1.39264$

$-0.133847$

0.980979

Y

Z

1.498202

0.351544

$-0.15197$

$-1.220151$

0.392666

$-0.099336$

$-1.29389$

1.59651

0.059104

$-0.951797$

$-1.482166$

2.01831

$-0.997291$

0.948842

2.421854

$-1.818036$

1.630013

3.017588

0.166881

$-1.158903$

3.961461

2.033321

0.031868

$-1.782985$

1.57112

1.104649

2.334773

1.888476

$-1.410291$

1.619146 
O

C

O

C

O

C

$\mathrm{H}$

$\mathrm{H}$

C

$\mathrm{H}$

$\mathrm{H}$

$\mathrm{H}$

$\mathrm{H}$

H

$\mathrm{TS}_{13}$

Symbol

$\mathrm{Fe}$

$\mathrm{Fe}$

S

S

C

O

C

$\mathrm{O}$

C

O

C

O

C

O

C

O

C

$\mathrm{H}$

$\mathrm{H}$

C

$\mathrm{H}$

H

$\mathrm{H}$

$\mathrm{H}$

H

$\begin{array}{rrr}-1.618617 & 2.35359 & -2.286394 \\ -2.88192 & -0.26847 & -0.418028 \\ -3.920962 & -0.71124 & -0.584127 \\ -1.70229 & 1.602735 & 1.182783 \\ -1.996887 & 2.351304 & 1.991516 \\ -1.044578 & -2.738163 & 0.446551 \\ -0.805462 & -3.608996 & 1.064321 \\ -2.051925 & -2.872523 & 0.048003 \\ -0.039319 & -2.632742 & -0.70054 \\ -0.345865 & -3.304922 & -1.50506 \\ 0.968113 & -2.920677 & -0.400947 \\ 1.375296 & 1.564524 & -1.283729 \\ 0.063252 & -0.179297 & 0.946023 \\ 0.695239 & 1.626063 & -0.825136\end{array}$

X

$-1.373996$

1.301798

0.493634

0.004018

$-1.466472$

$-2.272686$

$-2.918283$

$-3.877911$

$-2.166712$

$-2.642153$

1.658012

1.853992

2.818132

3.76589

2.023346

2.450188

0.50817

$-0.03595$

1.556516

$-0.090195$

0.442521

$-1.144643$

$-1.585605$

0.004122

$-1.736775$
Y

$-0.457841$

$-0.342488$

1.146314

0.888582

0.693051

1.218951

0.194379

0.614943

$-1.800738$

$-2.648429$

$-1.556233$

$-2.319176$

0.632781

1.234213

$-1.388316$

$-2.041957$

2.713681

3.455424

3.01547

2.590439

3.248495

2.870601

$-1.690719$

$-1.240463$

$-2.351195$
Z

$-0.250994$

$-0.035478$

1.590446

$-1.509764$

1.208534

1.888384

$-0.95147$

$-1.402457$

0.69269

1.290351

$-1.358039$

$-2.18027$

$-0.343306$

$-0.540512$

1.275384

2.105244

0.62534

1.215613

0.581825

$-0.781356$

$-1.470246$

$-0.796415$

$-2.74735$

0.314188

$-2.432835$

$\mathrm{TS}_{14}$ 


\begin{tabular}{|c|c|c|c|}
\hline Symbol & $\mathrm{X}$ & $\mathrm{Y}$ & $\mathrm{Z}$ \\
\hline $\mathrm{Fe}$ & 1.540021 & -0.076818 & -0.196292 \\
\hline $\mathrm{Fe}$ & -0.974663 & 0.543044 & -0.11979 \\
\hline$S$ & -2.099222 & -1.054137 & 1.293651 \\
\hline$S$ & -0.091569 & -1.25365 & -1.307236 \\
\hline $\mathrm{C}$ & 0.831748 & -0.064317 & 1.414366 \\
\hline $\mathrm{O}$ & 0.706603 & -0.188966 & 2.562428 \\
\hline $\mathrm{C}$ & 2.765674 & -1.397469 & -0.157355 \\
\hline $\mathrm{O}$ & 3.496292 & -2.269613 & -0.027874 \\
\hline $\mathrm{C}$ & 2.716499 & 1.224676 & 0.293275 \\
\hline $\mathrm{O}$ & 3.460365 & 2.049231 & 0.563386 \\
\hline $\mathrm{C}$ & -0.010235 & 1.704752 & -1.104846 \\
\hline $\mathrm{O}$ & 0.491387 & 2.50313 & -1.762347 \\
\hline $\mathrm{C}$ & -2.468097 & 0.712875 & -1.146776 \\
\hline $\mathrm{O}$ & -3.389893 & 0.82931 & -1.809918 \\
\hline $\mathrm{C}$ & -1.346512 & 1.848263 & 1.100601 \\
\hline $\mathrm{O}$ & -1.595418 & 2.653038 & 1.871083 \\
\hline $\mathrm{C}$ & -2.046454 & -2.557697 & 0.208117 \\
\hline $\mathrm{H}$ & -2.333384 & -3.410372 & 0.828301 \\
\hline $\mathrm{H}$ & -2.77025 & -2.43071 & -0.597889 \\
\hline $\mathrm{C}$ & -0.634248 & -2.730792 & -0.318591 \\
\hline $\mathrm{H}$ & -0.603871 & -3.581962 & -1.002633 \\
\hline $\mathrm{H}$ & 0.081465 & -2.906477 & 0.485125 \\
\hline $\mathrm{H}$ & 2.687469 & 0.072467 & -2.557864 \\
\hline $\mathrm{H}$ & -3.403222 & -0.753224 & 1.137412 \\
\hline $\mathrm{H}$ & 2.490176 & 0.762202 & -2.763764 \\
\hline \multicolumn{4}{|l|}{$\mathbf{T S}_{15}$} \\
\hline Symbol & $\mathrm{X}$ & $\mathrm{Y}$ & $\mathrm{Z}$ \\
\hline $\mathrm{Fe}$ & -1.537164 & 0.126861 & -0.237718 \\
\hline $\mathrm{Fe}$ & 0.979844 & -0.58232 & -0.10363 \\
\hline$S$ & 1.621216 & 1.160254 & 1.376399 \\
\hline$S$ & 0.134624 & 1.233207 & -1.286276 \\
\hline $\mathrm{C}$ & -1.049765 & -0.257246 & 1.40755 \\
\hline $\mathrm{O}$ & -1.016943 & -0.419067 & 2.554564 \\
\hline $\mathrm{C}$ & -2.686319 & 1.507763 & -0.091299 \\
\hline $\mathrm{O}$ & -3.366646 & 2.404645 & 0.118005 \\
\hline $\mathrm{C}$ & -2.824929 & -1.153498 & -0.14977 \\
\hline $\mathrm{O}$ & -3.634363 & -1.960905 & -0.159113 \\
\hline $\mathrm{C}$ & 0.130275 & -1.698442 & -1.230992 \\
\hline $\mathrm{O}$ & -0.341089 & -2.429596 & -1.979877 \\
\hline $\mathrm{C}$ & 2.594113 & -0.647467 & -0.943631 \\
\hline $\mathrm{O}$ & 3.594333 & -0.710945 & -1.489817 \\
\hline $\mathrm{C}$ & 1.316181 & -1.915186 & 1.092728 \\
\hline
\end{tabular}


O

C

H

$\mathrm{H}$

C

$\mathrm{H}$

$\mathrm{H}$

$\mathrm{H}$

Species $\mathrm{H}^{+}\left(\mathrm{SiEt}_{3}\right)_{2}$

Symbol

$\mathrm{Si}$

$\mathrm{H}$

C

$\mathrm{H}$

$\mathrm{H}$

C

$\mathrm{H}$

$\mathrm{H}$

C

$\mathrm{H}$

$\mathrm{H}$

C

$\mathrm{H}$

$\mathrm{H}$

$\mathrm{H}$

C

$\mathrm{H}$

$\mathrm{H}$

$\mathrm{H}$

C

$\mathrm{H}$

H

$\mathrm{H}$

$\mathrm{Si}$

C

$\mathrm{H}$

$\mathrm{H}$

C

$\mathrm{H}$

$\mathrm{H}$

C

$\mathrm{H}$

H

$\begin{array}{lll}1.555659 & -2.737152 & 1.848062 \\ 2.133006 & 2.524851 & 0.276315 \\ 2.371825 & 3.398482 & 0.886819 \\ 3.000363 & 2.239583 & -0.31974 \\ 0.891964 & 2.792294 & -0.577536 \\ 1.157162 & 3.399764 & -1.444162 \\ 0.116159 & 3.296754 & -0.001597 \\ 2.396613 & 1.117741 & 2.476961\end{array}$

Z

$\begin{array}{lll}-1.753892 & 0.180825 & -0.034915\end{array}$

$\begin{array}{lll}-0.113623 & 0.114388 & 0.085264\end{array}$

$\begin{array}{lll}-2.286732 & -1.376905 & 0.869976\end{array}$

$\begin{array}{lll}-1.90601 & -1.324304 & 1.896976\end{array}$

$\begin{array}{lll}-3.379024 & -1.281592 & 0.963234\end{array}$

$\begin{array}{lll}-1.996232 & 0.214671 & -1.898848\end{array}$

$\begin{array}{lll}-1.498513 & 1.109508 & -2.291782\end{array}$

$\begin{array}{lll}-1.484805 & -0.646339 & -2.344233\end{array}$

$\begin{array}{lll}-2.171117 & 1.78845 & 0.841237\end{array}$

$\begin{array}{lll}-1.642166 & 2.605394 & 0.336375\end{array}$

$\begin{array}{lll}-3.236635 & 1.957292 & 0.624951\end{array}$

$\begin{array}{lll}-1.930252 & -2.728602 & 0.227721\end{array}$

$\begin{array}{lll}-2.314762 & -2.810424 & -0.793191\end{array}$

$\begin{array}{lll}-0.84831 & -2.888099 & 0.191848\end{array}$

$\begin{array}{lll}-2.361023 & -3.550554 & 0.807252\end{array}$

$\begin{array}{lll}-3.48519 & 0.214109 & -2.31256\end{array}$

$\begin{array}{lll}-4.018841 & 1.08371 & -1.917277\end{array}$

$\begin{array}{lll}-3.572809 & 0.243856 & -3.402641\end{array}$

$\begin{array}{lll}-4.003744 & -0.685098 & -1.966028\end{array}$

$\begin{array}{lll}-1.936105 & 1.840537 & 2.361067\end{array}$

$\begin{array}{lll}-2.262169 & 2.803762 & 2.764643\end{array}$

$\begin{array}{lll}-2.494888 & 1.059006 & 2.884098\end{array}$

$\begin{array}{lll}-0.878455 & 1.720956 & 2.615552\end{array}$

$\begin{array}{lll}1.526212 & 0.164801 & -0.020631\end{array}$

$\begin{array}{lll}1.814785 & 1.946383 & -0.553717\end{array}$

$\begin{array}{lll}1.350877 & 2.606174 & 0.188994\end{array}$

$\begin{array}{lll}1.281151 & 2.113598 & -1.497348\end{array}$

$\begin{array}{lll}2.041063 & -0.242287 & 1.740412\end{array}$

$\begin{array}{lll}3.121382 & -0.039613 & 1.781469\end{array}$

$\begin{array}{lll}1.583387 & 0.494522 & 2.411455\end{array}$

$\begin{array}{lll}1.852564 & -1.173169 & -1.3045\end{array}$

$1.253098 \quad-2.053584 \quad-1.045267$

$\begin{array}{lll}1.480288 & -0.821575 & -2.273933\end{array}$ 


$\begin{array}{rrr}3.339012 & -1.577692 & -1.420543 \\ 3.728184 & -1.969581 & -0.476087 \\ 3.456605 & -2.361553 & -2.174622 \\ 3.971887 & -0.737505 & -1.720044 \\ 3.305363 & 2.31663 & -0.72158 \\ 3.789137 & 1.719467 & -1.500019 \\ 3.400088 & 3.367738 & -1.009949 \\ 3.86753 & 2.180141 & 0.207106 \\ 1.760439 & -1.674705 & 2.228905 \\ 2.141432 & -1.813482 & 3.245054 \\ 0.689419 & -1.89741 & 2.248048 \\ 2.243257 & -2.422078 & 1.592313\end{array}$

Species $\left(\mathrm{SiEt}_{3}\right)_{2}$

$\begin{array}{lrrr}\text { Symbol } & \mathrm{X} & \mathrm{Y} & \mathrm{Z} \\ \mathrm{Si} & -1.298921 & 0.165898 & -0.055536 \\ \mathrm{C} & -2.133577 & -1.219484 & 0.969545 \\ \mathrm{H} & -1.84141 & -1.101105 & 2.021007 \\ \mathrm{H} & -3.21548 & -1.024152 & 0.944745 \\ \mathrm{C} & -1.903803 & 0.046195 & -1.870604 \\ \mathrm{H} & -1.426414 & 0.855246 & -2.440177 \\ \mathrm{H} & -1.512295 & -0.883917 & -2.303678 \\ \mathrm{C} & -1.899351 & 1.841119 & 0.657334 \\ \mathrm{H} & -1.509173 & 2.646935 & 0.020963 \\ \mathrm{H} & -2.991269 & 1.876017 & 0.538712 \\ \mathrm{C} & -1.864901 & -2.664359 & 0.517542 \\ \mathrm{H} & -2.152804 & -2.821079 & -0.527811 \\ \mathrm{H} & -0.804169 & -2.922475 & 0.604429 \\ \mathrm{H} & -2.425171 & -3.388081 & 1.121199 \\ \mathrm{C} & -3.428756 & 0.10342 & -2.072562 \\ \mathrm{H} & -3.851423 & 1.043385 & -1.701609 \\ \mathrm{H} & -3.698815 & 0.022289 & -3.132227 \\ \mathrm{H} & -3.936372 & -0.711757 & -1.545395 \\ \mathrm{C} & -1.540029 & 2.124711 & 2.126037 \\ \mathrm{H} & -1.944084 & 3.086879 & 2.462412 \\ \mathrm{H} & -1.937802 & 1.354549 & 2.795782 \\ \mathrm{H} & -0.455758 & 2.158142 & 2.278905 \\ \mathrm{Si} & 1.085169 & 0.092349 & -0.106097 \\ \mathrm{C} & 1.636946 & 1.739072 & -0.925301 \\ \mathrm{H} & 1.054431 & 2.54522 & -0.458731 \\ \mathrm{H} & 1.308749 & 1.716504 & -1.973862 \\ \mathrm{H} & 1.898861 & -0.019235 & 1.623503 \\ \mathrm{H} & 2.985659 & 0.060043 & 1.482709 \\ \mathrm{H} & 1.614421 & 0.871695 & 2.198864\end{array}$




$\begin{array}{lrrr}\mathrm{C} & 1.638405 & -1.41606 & -1.154215 \\ \mathrm{H} & 1.147449 & -2.30648 & -0.739093 \\ \mathrm{H} & 1.216962 & -1.298956 & -2.16187 \\ \mathrm{C} & 3.152628 & -1.67627 & -1.253984 \\ \mathrm{H} & 3.610831 & -1.784004 & -0.264762 \\ \mathrm{H} & 3.365776 & -2.595366 & -1.812857 \\ \mathrm{H} & 3.671753 & -0.85941 & -1.765105 \\ \mathrm{C} & 3.131167 & 2.105078 & -0.860468 \\ \mathrm{H} & 3.758345 & 1.360854 & -1.361002 \\ \mathrm{H} & 3.326393 & 3.070538 & -1.342607 \\ \mathrm{H} & 3.4824 & 2.183474 & 0.173786 \\ \mathrm{C} & 1.585655 & -1.284281 & 2.440652 \\ \mathrm{H} & 2.109883 & -1.283218 & 3.403589 \\ \mathrm{H} & 0.515164 & -1.373417 & 2.65319 \\ \mathrm{H} & 1.887286 & -2.193239 & 1.908491\end{array}$

Species HOTf

Symbol

$\mathrm{S}$

F

F

F

O

O

$\mathrm{O}$

C

$\mathrm{H}$

Species OTf ${ }^{\square}$

Symbol

$\mathrm{S}$

F

F

F

O

$\mathrm{O}$

O

C

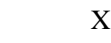

0.85202

$-1.414535$

$-1.379246$

$-1.537151$

1.255095

1.218905

1.270645

$-1.011823$

1.459857

$\begin{array}{rr}\mathrm{Y} & \mathrm{Z} \\ -0.142393 & 0.061827 \\ 0.012204 & -1.267842 \\ 1.14557 & 0.592035 \\ -1.026247 & 0.64238 \\ -0.012055 & 1.45557 \\ -1.283339 & -0.757192 \\ 1.183748 & -0.764851 \\ 0.01216 & -0.001649 \\ 1.914758 & -0.146716\end{array}$

Z

267842

.592035

.757192

$-0.764851$

0.146716

$\begin{array}{rrr}\mathrm{X} & \mathrm{Y} & \mathrm{Z} \\ -0.909567 & -0.000073 & 0.000029 \\ 1.434655 & -0.947028 & -0.824154 \\ 1.434575 & -0.240093 & 1.232414 \\ 1.43411 & 1.187492 & -0.408169 \\ -1.245074 & 1.095339 & 0.940604 \\ -1.244555 & 0.266884 & -1.418965 \\ -1.244693 & -1.362504 & 0.478146 \\ 0.949597 & 0.000012 & 0.000074\end{array}$


Table S10. Cartesian coordinates for DFT geometry-optimized species using BP86.

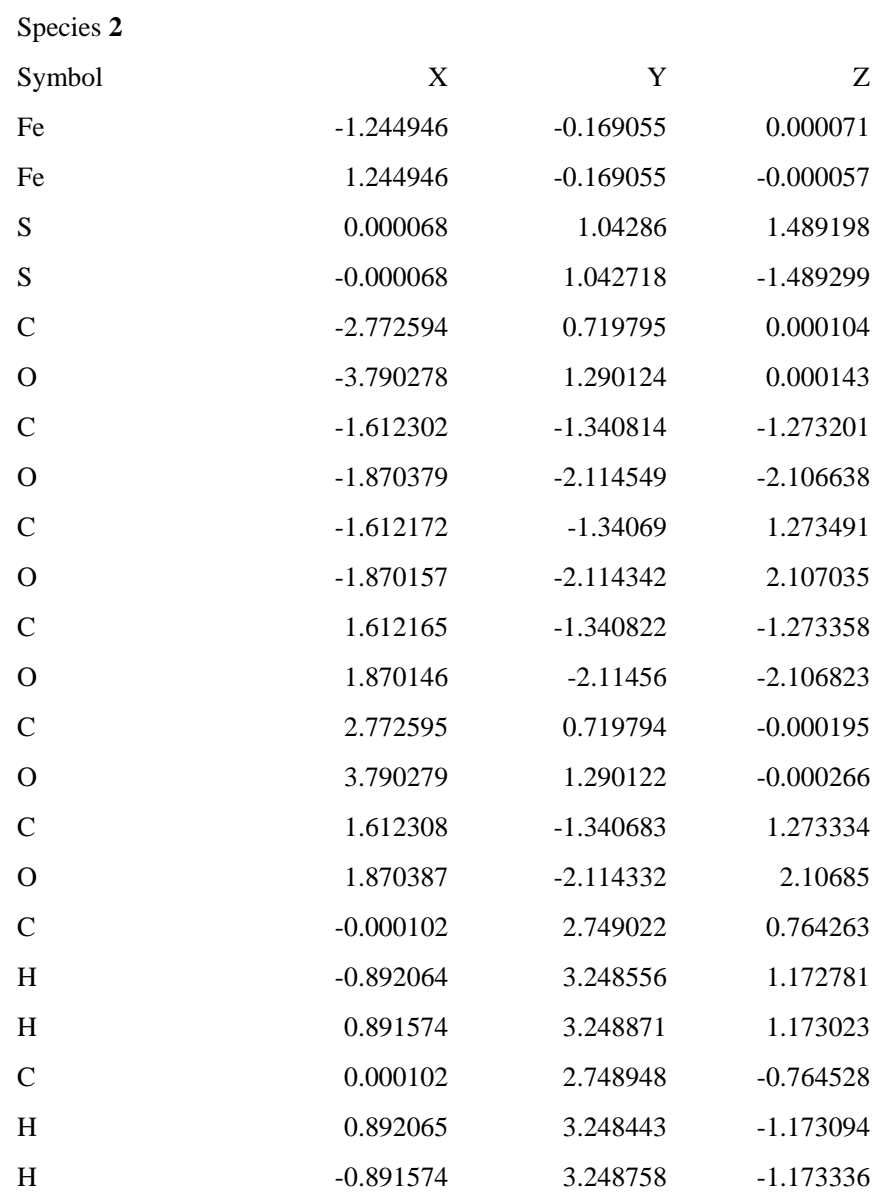

Species $\mathbf{2} \boldsymbol{\mu} \mathbf{H}^{+}$

$\begin{array}{lrrr}\text { Symbol } & \mathrm{X} & \mathrm{Y} & \mathrm{Z} \\ \mathrm{Fe} & -1.275671 & -0.248127 & 0.00154 \\ \mathrm{Fe} & 1.275668 & -0.248142 & -0.001541 \\ \mathrm{~S} & 0.002555 & 0.944722 & 1.495721 \\ \mathrm{~S} & -0.002546 & 0.944712 & -1.495732 \\ \mathrm{C} & -2.687254 & 0.83201 & -0.001085 \\ \mathrm{O} & -3.613507 & 1.525042 & -0.004937 \\ \mathrm{C} & -1.902982 & -1.302243 & -1.306445 \\ \mathrm{O} & -2.301577 & -1.988002 & -2.146751 \\ \mathrm{C} & -1.904387 & -1.297573 & 1.312863 \\ \mathrm{O} & -2.306041 & -1.980094 & 2.154341 \\ \mathrm{C} & 1.904384 & -1.297603 & -1.312853 \\ \mathrm{O} & 2.30604 & -1.980134 & -2.154322 \\ \mathrm{C} & 2.68724 & 0.832009 & 0.001085 \\ \mathrm{O} & 3.613471 & 1.52507 & 0.004947 \\ \mathrm{C} & 1.902977 & -1.30225 & 1.306449\end{array}$




$\begin{array}{lrrr}\mathrm{O} & 2.301574 & -1.988004 & 2.146759 \\ \mathrm{C} & 0.017352 & 2.643332 & 0.764822 \\ \mathrm{H} & -0.852618 & 3.162041 & 1.195459 \\ \mathrm{H} & 0.926049 & 3.126394 & 1.155722 \\ \mathrm{C} & -0.017304 & 2.643326 & -0.764845 \\ \mathrm{H} & 0.85268 & 3.162008 & -1.195486 \\ \mathrm{H} & -0.925986 & 3.126411 & -1.15575 \\ \mathrm{H} & -0.000006 & -1.32186 & 0.000003\end{array}$

\begin{tabular}{|c|c|c|c|}
\hline Species & & & \\
\hline Symbol & $\mathrm{X}$ & $\mathrm{Y}$ & $\mathrm{Z}$ \\
\hline $\mathrm{Fe}$ & 1.363095 & -0.245781 & -0.000684 \\
\hline $\mathrm{Fe}$ & -1.363106 & -0.245785 & 0.000686 \\
\hline$S$ & -0.00233 & 0.911816 & -1.519872 \\
\hline$S$ & 0.002321 & 0.911836 & 1.519861 \\
\hline $\mathrm{C}$ & 2.759318 & 0.909509 & -0.002358 \\
\hline $\mathrm{O}$ & 3.715013 & 1.575696 & -0.002916 \\
\hline $\mathrm{C}$ & 1.95455 & -1.301625 & 1.289129 \\
\hline $\mathrm{O}$ & 2.347657 & -1.991452 & 2.142326 \\
\hline $\mathrm{C}$ & 1.953901 & -1.301514 & -1.291037 \\
\hline $\mathrm{O}$ & 2.346112 & -1.991899 & -2.144173 \\
\hline $\mathrm{C}$ & -1.9539 & -1.301508 & 1.291054 \\
\hline $\mathrm{O}$ & -2.346109 & -1.991879 & 2.144204 \\
\hline $\mathrm{C}$ & -2.759344 & 0.909501 & 0.002339 \\
\hline $\mathrm{O}$ & -3.715014 & 1.57573 & 0.002878 \\
\hline $\mathrm{C}$ & -1.954529 & -1.301682 & -1.289105 \\
\hline $\mathrm{O}$ & -2.34761 & -1.991541 & -2.142288 \\
\hline $\mathrm{C}$ & -0.015881 & 2.602486 & -0.768424 \\
\hline $\mathrm{H}$ & 0.856516 & 3.130541 & -1.184568 \\
\hline $\mathrm{H}$ & -0.924366 & 3.096107 & -1.147774 \\
\hline $\mathrm{C}$ & 0.015882 & 2.602497 & 0.768392 \\
\hline $\mathrm{H}$ & -0.856512 & 3.130561 & 1.184529 \\
\hline $\mathrm{H}$ & 0.924371 & 3.096118 & 1.147734 \\
\hline $\mathrm{H}$ & 0.000046 & -1.234279 & 0.000023 \\
\hline
\end{tabular}

Species $\mathbf{2} \boldsymbol{\mu} \mathbf{H}^{*-}$

$\begin{array}{lrrr}\text { Symbol } & \mathrm{X} & \mathrm{Y} & \mathrm{Z} \\ \mathrm{Fe} & 1.14987 & -0.430634 & -0.031894 \\ \mathrm{Fe} & -1.390403 & -0.035252 & 0.067695 \\ \mathrm{~S} & 0.034225 & 0.986941 & -1.464188 \\ \mathrm{~S} & 2.283011 & 1.483965 & 0.919918 \\ \mathrm{C} & 2.449462 & -0.848133 & -1.114975 \\ \mathrm{O} & 3.326909 & -1.131276 & -1.840879 \\ \mathrm{C} & 1.765858 & -1.341116 & 1.324833\end{array}$




$\begin{array}{lrrr}\mathrm{O} & 2.183259 & -1.937245 & 2.244027 \\ \mathrm{C} & -0.088257 & -1.67374 & -0.554913 \\ \mathrm{O} & -0.350475 & -2.767935 & -0.953708 \\ \mathrm{C} & -2.122448 & 1.450404 & 0.720571 \\ \mathrm{O} & -2.739464 & 2.359928 & 1.131442 \\ \mathrm{C} & -2.550704 & -0.497015 & -1.180224 \\ \mathrm{O} & -3.351255 & -0.788259 & -1.985407 \\ \mathrm{C} & -2.013938 & -1.099353 & 1.310366 \\ \mathrm{O} & -2.413143 & -1.797432 & 2.162893 \\ \mathrm{H} & -0.089325 & 0.158885 & 1.043621 \\ \mathrm{C} & 0.518247 & 2.67875 & -0.878179 \\ \mathrm{H} & 1.341188 & 2.977163 & -1.549893 \\ \mathrm{H} & -0.343148 & 3.344405 & -1.063451 \\ \mathrm{C} & 0.967148 & 2.719671 & 0.576945 \\ \mathrm{H} & 0.105488 & 2.536183 & 1.239142 \\ \mathrm{H} & 1.365023 & 3.722841 & 0.814591\end{array}$

\section{Species $\mathbf{2} \boldsymbol{\mu} \mathbf{H S H}^{*}$}

\begin{tabular}{|c|c|c|c|}
\hline Symbol & $\mathrm{X}$ & $\mathrm{Y}$ & $\mathrm{Z}$ \\
\hline $\mathrm{Fe}$ & 1.145159 & -0.435023 & 0.002984 \\
\hline $\mathrm{Fe}$ & -1.367774 & -0.048136 & 0.05981 \\
\hline S & 0.071788 & 0.991995 & -1.449167 \\
\hline S & 2.196861 & 1.474102 & 1.034854 \\
\hline $\mathrm{C}$ & 2.419998 & -0.893908 & -1.105875 \\
\hline $\mathrm{O}$ & 3.259433 & -1.217202 & -1.852977 \\
\hline $\mathrm{C}$ & 1.685096 & -1.525972 & 1.270527 \\
\hline $\mathrm{O}$ & 2.037815 & -2.248783 & 2.117215 \\
\hline $\mathrm{C}$ & -0.170489 & -1.613115 & -0.582792 \\
\hline $\mathrm{O}$ & -0.371619 & -2.692333 & -1.047677 \\
\hline $\mathrm{C}$ & -2.082467 & 1.443503 & 0.756877 \\
\hline $\mathrm{O}$ & -2.645958 & 2.364479 & 1.20053 \\
\hline $\mathrm{C}$ & -2.569846 & -0.392074 & -1.194649 \\
\hline $\mathrm{O}$ & -3.377903 & -0.608089 & -2.006888 \\
\hline $\mathrm{C}$ & -2.063564 & -1.126681 & 1.263751 \\
\hline $\mathrm{O}$ & -2.510305 & -1.831575 & 2.07835 \\
\hline $\mathrm{H}$ & -0.098515 & 0.11171 & 1.08855 \\
\hline $\mathrm{C}$ & 0.550369 & 2.691286 & -0.886931 \\
\hline $\mathrm{H}$ & 1.36009 & 2.993087 & -1.571811 \\
\hline $\mathrm{H}$ & -0.310704 & 3.35548 & -1.06888 \\
\hline $\mathrm{C}$ & 1.001353 & 2.798359 & 0.561865 \\
\hline $\mathrm{H}$ & 0.16484 & 2.666263 & 1.262669 \\
\hline $\mathrm{H}$ & 1.468257 & 3.775094 & 0.764146 \\
\hline $\mathrm{H}$ & 3.15124 & 1.782563 & 0.116619 \\
\hline
\end{tabular}




$\begin{array}{lrrr}\text { Species } 2 \mu \mathbf{H t H}^{\mathrm{S}} \mathbf{S H}^{*+} & & & \\ \text { Symbol } & \mathrm{X} & \mathrm{Y} & \mathrm{Z} \\ \mathrm{Fe} & 1.218355 & -0.377287 & 0.026426 \\ \mathrm{Fe} & -1.489938 & -0.046121 & 0.127336 \\ \mathrm{~S} & -0.075009 & 0.80804 & -1.438668 \\ \mathrm{~S} & 1.445464 & 1.583319 & 1.354219 \\ \mathrm{C} & 2.753251 & -0.023546 & -0.815205 \\ \mathrm{O} & 3.749705 & 0.187544 & -1.362831 \\ \mathrm{C} & 1.90019 & -1.309562 & 1.402154 \\ \mathrm{O} & 2.324152 & -1.915257 & 2.290141 \\ \mathrm{C} & 0.977868 & -1.860436 & -0.925812 \\ \mathrm{O} & 0.853886 & -2.833326 & -1.537784 \\ \mathrm{C} & -2.831584 & 1.034545 & -0.223516 \\ \mathrm{O} & -3.695661 & 1.7832 & -0.42139 \\ \mathrm{C} & -2.027742 & -1.406669 & -0.957678 \\ \mathrm{O} & -2.48563 & -2.248526 & -1.609215 \\ \mathrm{C} & -2.192769 & -0.722344 & 1.603621 \\ \mathrm{O} & -2.627608 & -1.13315 & 2.596948 \\ \mathrm{H} & -0.159438 & -0.705585 & 0.890383 \\ \mathrm{C} & 0.413332 & 2.557426 & -1.075274 \\ \mathrm{H} & 1.364193 & 2.704568 & -1.611188 \\ \mathrm{H} & -0.348926 & 3.197742 & -1.550047 \\ \mathrm{C} & 0.534944 & 2.890284 & 0.395947 \\ \mathrm{H} & -0.445196 & 2.959453 & 0.886627 \\ \mathrm{H} & 1.07774 & 3.834372 & 0.559255 \\ \mathrm{H} & 2.702727 & 1.992977 & 1.033624 \\ & -1.172918 & 1.081277 & 1.072344\end{array}$




\section{References}

(1) Surawatanawong, P.; Tye, J. W.; Darensbourg, M. Y.; Hall, M. B. Dalton Trans. 2010, 39, 3093-3104.

(2) Skagestad, V.; Tilset, M. J. Am. Chem. Soc. 1993, 115, 5077-5083.

(3) Ciancanelli, R.; Noll, B. C.; DuBois, D. L.; DuBois, M. R. J. Am. Chem. Soc. 2002, 124, 2984-2992.

(4) Keith, J. A.; Carter, E. A. J. Am. Chem. Soc. 2012, 134, 7580-7583.

(5) Hughes, T. F.; Friesner, R. A. J. Phys. Chem. B 2011, 115, 9280-9289.

(6) Felton, G. A. N.; Petro, B. J.; Glass, R. S.; Lichtenberger, D. L.; Evans, D. H. J. Am. Chem. Soc. 2009, 131, 11290-11291.

(7) Namazian, M.; Lin, C. Y.; Coote, M. L. J. Chem. Theory Comput. 2010, 6, 2721-2725. 